\title{
Observation of Odderon effects at LHC energies: a real extended Bialas-Bzdak model study
}

\author{
T. Csörgö ${ }^{1,2, a}$, I. Szanyi ${ }^{1,3, b}$ \\ ${ }^{1}$ Wigner FK, POB 49, Budapest 114 1525, Hungary \\ ${ }^{2}$ MATE Institute of Technology, Károly Róbert Campus, Mátrai út 36, Gyöngyös 3200, Hungary \\ ${ }^{3}$ Eötvös University, Pázmány P. s. 1/A, Budapest 1117, Hungary
}

Received: 6 August 2020 / Accepted: 25 June 2021 / Published online: 13 July 2021

(C) The Author(s) 2021

\begin{abstract}
The unitarily extended Bialas-Bzdak model of elastic proton-proton scattering is applied, without modifications, to describe the differential cross-section of elastic proton-antiproton collisions in the $\mathrm{TeV}$ energy range, and to extrapolate these differential cross-sections to LHC energies. In this model-dependent study we find that the differential cross-sections of elastic proton-proton collision data at 2.76 and $7 \mathrm{TeV}$ energies differ significantly from the differential cross-section of elastic proton-antiproton collisions extrapolated to these energies. The elastic proton-proton differential cross-sections, extrapolated to $1.96 \mathrm{TeV}$ energy with the help of this extended Bialas-Bzdak model do not differ significantly from that of elastic proton-antiproton collisions, within the theoretical errors of the extrapolation. Taken together these results provide a model-dependent, but statistically significant evidence for a crossing-odd component of the elastic scattering amplitude at the at least 7.08 sigma level. From the reconstructed Odderon and Pomeron amplitudes, we determine the $\sqrt{s}$ dependence of the corresponding total and differential cross-sections.
\end{abstract}

\section{Introduction}

Recently the TOTEM experiment measured differential cross-sections of elastic proton-proton collisions in the $\mathrm{TeV}$ energy range, from $\sqrt{s}=2.76$ through 7 and 8 to $13 \mathrm{TeV}$, together with the total, elastic and inelastic cross-sections and the real to imaginary ratio of the scattering amplitude at vanishing four-momentum transfer. These measurements provided surprizes and unexpected results. First of all, the shape of the differential cross-section of elastic scattering at $\sqrt{s}=7 \mathrm{TeV}$ was different from all the predictions.

\footnotetext{
a e-mail: tcsorgo@cern.ch (corresponding author)

b e-mail: istvan.szanyi@cern.ch
}

The total cross-section increases with increasing $\sqrt{s}$ according to theoretical expectations based on Pomeronexchange, corresponding experimentally to the production of large rapidity gaps in high energy proton-proton and protonantiproton collisions. These events correspond to large angular regions where no particle is produced. Their fraction, in particular the ratio of the elastic to the total proton-proton cross-section is increased above $25 \%$ at LHC energies.

In the language of quantum chromodynamics (QCD), the field theory of strong interactions, Pomeron-exchange corresponds to the exchange of even number of gluons with vacuum quantum numbers. In 1973, a crossing-odd counterpart to the Pomeron was proposed by Lukaszuk and Nicolescu, the so-called Odderon [1]. In QCD, Odderon exchange corresponds to the $t$-channel exchange of a color-neutral gluonic compound state consisting of an odd number of gluons, as noted by Bartels, Vacca and Lipatov in 1999 [2].

The Odderon effects remained elusive for a long time, due to lack of a definitive and statistically significant experimental evidence.

A direct way to probe the Odderon in elastic scattering is by comparing the differential cross-section of particleparticle and particle-antiparticle scattering at sufficiently high energies $[3,4]$. Such a search was published at the ISR energy of $\sqrt{s}=53 \mathrm{GeV}$ in 1985 [5], that resulted in an indication of the Odderon, corresponding to a $3.35 \sigma$ significance level obtained from a simple $\chi^{2}$ calculation, based on $5 p p$ and $5 p \bar{p}$ data points in the $1.1 \leq|t| \leq 1.5 \mathrm{GeV}^{2}$ range (around the diffractive minimum). This significance is smaller than the $5 \sigma$ threshold, traditionally accepted as a threshold for a discovery level observation in high energy physics. Furthermore, the colliding energy of $\sqrt{s}=53 \mathrm{GeV}$ was not sufficiently large so the possible Reggeon exchange effects were difficult to evaluate and control. These difficulties rendered the Odderon search at the ISR energies rather 
inconclusive, but nevertheless inspiring and indicative, motivating further studies.

In a series of recent papers, the TOTEM Collaboration published results with important implications for the Odderon search. These papers studied elastic proton-proton collisions in the LHC energy range between $\sqrt{s}=2.76$ and $13 \mathrm{TeV}$ [6-9]. The total cross section, $\sigma_{\text {tot }}(s)$ was found to increase, while the real-to-imaginary ratio, $\rho_{0}(s)$, is found to decrease with increasing energy, first identified at $\sqrt{s}=13$ $\mathrm{TeV}[6,7]$. These experimental results at vanishing fourmomentum transfer were consistent with a possible Odderon effect and triggered an intense theoretical debate (see e.g. Refs. [10-27]). For example, Ref. [28] demonstrated that such an indication at $t=0$ is not a unique Odderon signal, as such a behaviour can be attributed to secondary Reggeon effects. In spite of the rich experimental results and the hot theoretical debate, the Odderon remained rather elusive at vanishing four-momentum transfer even in the $\mathrm{TeV}$ energy range [29].

However, at larger four-momentum transfers, in the interference (diffractive dip and bump or minimum-maximum) region, the Odderon signals are significant at LHC energies. Let us mention here only two of them: the four-momentum transfer dependent nuclear slope parameter $B(t)$ and the scaling properties of elastic scattering at the $\mathrm{TeV}$ energy region.

Two independent, but nearly simultaneous phenomenological papers suggested that the four-momentum transfer dependence of the nuclear slope parameter, $B(t)$ is qualitatively different in elastic proton-proton and protonantiproton collisions $[12,22]$. The TOTEM experiment has demonstrated in Ref. [9] that indeed in elastic $p p$ collisions at $\sqrt{s}=2.76 \mathrm{TeV}$, the nuclear slope $B(t)$ is increasing (swings) before it decreases and changes sign in the interference (diffractive dip and bump or minimum-maximum) region. After the diffractive maximum, the nuclear slope becomes positive again. In contrast, elastic $p \bar{p}$ collisions measured by the D0 collaboration at the Tevatron energy of $\sqrt{s}=1.96 \mathrm{TeV}$ did not show such a pronounced diffractive minimum-maximum structure, instead an exponentially decreasing cone region at low $-t$ with a constant $B(t)$ is followed by a shoulder structure, without a pronounced diffractive minimum and maximum structure. The TOTEM collaboration presented its results on the elastic $p p$ differential cross-section at $\sqrt{s}=2.76 \mathrm{TeV}$ and concluded in Ref. [9] that "under the condition that the effects due to the energy difference between TOTEM and D0 can be neglected, these results provide evidence for a colourless 3 -gluon bound state exchange in the t-channel of the proton-proton elastic scattering".

This energy gap has been closed recently, in a modelindependent way, based on a re-analysis of already published data using the scaling properties of elastic scattering in both $p p$ and $p \bar{p}$ collisions at $\mathrm{TeV}$ energies: Refs. [30-32] reported about a statistically significant Odderon signal in the comparison of the $H(x, s)$ scaling functions of elastic $p p$ collisions at $\sqrt{s}=7.0 \mathrm{TeV}$ to that of $p \bar{p}$ collisions at $\sqrt{s}=1.96 \mathrm{TeV}$. The difference between these scaling functions carries an at least $6.26 \sigma$ Odderon signal, if all the vertical and horizontal, point-to-point fluctuating and point-to-point correlated errors are taken into account. If the interpolation between the datapoints at $7 \mathrm{TeV}$ is considered as a theoretical curve, the significance of the Odderon signal goes up to $6.55 \sigma$. Instead of comparing the cross sections directly, this method removes the dominant $s$ dependent quantities, by scaling out the $s$-dependencies of $\sigma_{\mathrm{tot}}(s), \sigma_{\mathrm{el}}(s), B(s)$ and $\rho_{0}(s)$, as well as the normalization of the $H(x, s)$ scaling function, that also cancels the point-to-point correlated and $t$-independent normalization errors.

The model-independence of the results of Refs. [12, 30-32] is an advantage when a significant and modelindependent Odderon signal is searched for. The domain of the signal region can also be determined with modelindependent methods. Both the signal and its domain can be directly determined from the comparison of D0 and TOTEM data. However, a physical interpretation or a theoretical context is also desired, not only to gain a better understanding of the results, in order to have a more physical picture, but also to gain a predictive power and to be able to extrapolate the results to domains where experimental data are lacking, or, to regions where the scaling relations are violated. To provide such a picture is one of the goals of our present manuscript. In this work, we continue a recent series of theoretical papers [33-36]. These studies investigated the differential cross-section of elastic $p p$ collisions, but did not study the same effects in elastic $p \bar{p}$ collisions. The framework of these studies is the real extended and unitarized BialasBzdak model, based on Refs. [37-40]. This model considers protons as weakly bound states of constituent quarks and diquarks, or $p=(q, d)$ for short (for a more detailed summary of the model see Appendix A). In a variation on this theme, the diquark in the proton may also be considered to be a weakly bound state of two constituent quarks, leading to the $p=(q,(q, q))$ variant of the Bialas-Bzdak model [37,38]. The model is based on Glauber's multiple scattering theory of elastic collisions [41-43], assuming additionally, that all elementary distributions follow a random Gaussian elementary process, and can be characterized by the corresponding $s$-dependent Gaussian radii. These distributions include the parton distribution inside the quark, characterized by a Gaussian radius $R_{q}(s)$, the distributions of the partons inside the diquarks, characterized by the Gaussian radius $R_{d}(s)$ and the typical separation between the quarks and the diquarks characterized by the Gaussian radius $R_{q d}(s)$. In Refs. $[33,34,36]$ it was shown that the $p=(q,(q, q))$ variant of the BialasBzdak model gives too many diffractive minima, while experimentally only a single diffractive minimum is observed in 
$p p$ collisions. This is a result that is consistent with the earlier detailed studies of elastic nucleus-nucleus collisions in Ref. [44], that observed that a single diffractive minimum occures only in elastic deuteron-deuteron or $(p, n)+(p, n)$ collisions, so the number of diffractive minima increases as either of the elastically colliding composite objects develops a more complex internal structure.

In the original version of the Bialas-Bzdak model, the scattering amplitude was assumed to be completely imaginary [37]. This structure resulted in a completely vanishing differential cross-section at the diffractive minima. This model was supplemented by a real part, first perturbatively [33-35], subsequently in a non-perturbative and unitary manner [36]. This way a new parameter called $\alpha(s)$ was introduced, that controls the value of the differential cross-section at the diffractive minimum (it is not to be confused with the strong coupling constant of QCD, that we denote in this work as $\alpha_{s}^{\mathrm{QCD}}$ ). Our $\alpha(s)$ is a kind of opacity parameter, that measures the strength of the real part of the scattering amplitude, so it is responsible for both for filling up the dip region of the differential cross-sections and for the description of the real to imaginary ratio $\rho$ at vanishing four-momentum transfer.

The structure of this unitary, real extended Bialas-Bzdak model (abbreviated as ReBB model) is thus very interesting as there are only four $s$-dependent physical parameters: $R_{q}$, $R_{d}, R_{q d}$ and $\alpha$. However three out of these four parameters is a geometrical parameter, characterizing the $s$ dependence of parton distributions inside the protons. Hence, it is natural to assume, that these distributions are the same inside protons and anti-protons, while the opacity parameter $\alpha$ may be different in elastic $p p$ and $p \bar{p}$ collisions.

So it is natural to expect, that this $\alpha(s)$ parameter may carry an Odderon signal as its excitation function might be very different in elastic $p p$ collisions, that feature a pronounced dip at every measured energy even in the $\mathrm{TeV}$ energy range [9], while in elastic $p \bar{p}$ collisions, a significant dip is lacking even in measurements in the TeV energy range [45].

In this manuscript, we thus extend the applications of the ReBB model from elastic $p p$ to elastic $p \bar{p}$ collisions using the model exactly in the same form, as it was described in Ref. [36]. We fit exactly the same four physical parameters to describe the differential cross-section of elastic protonantiproton $(p \bar{p})$ scattering. Later we shall see that at the same energy, the geometrical parameters in $p p$ and $p \bar{p}$ collisions are apparently consistent with one another, within the systematic errors of the analysis we obtain the same $R_{q}(s), R_{d}(s)$ and $R_{q d}(s)$ functions for $p p$ and $p \bar{p}$ reactions.

In this manuscript, we thus can investigate also the following independent questions:

- Is the real extended Bialas-Bzdak model of Ref. [36] able to describe not only elastic $p p$ but also $p \bar{p}$ collisions?
- Is it possible to characterize the Odderon with only one physical parameter: the difference of the opacity parameter $\alpha(s)$ in $p p$ and in $p \bar{p}$ collisions: $\alpha^{p p}(s) \neq \alpha^{p \bar{p}}(s)$ ?

We shall see that the answer to both of these questions is a definitive yes.

The structure of the manuscript is as follows. In Sect. 2 we recapitulate the definition of the key physical quantities in elastic scattering and mention their main relations. In Sect. 3 we present the various error definitions and the evaluated $\chi^{2}$ formulae of both $p p$ and $p \bar{p}$ datasets. Subsequently, in Sect. 4 we detail the optimization method and summarize the fit results in terms of four physical parameters determined at four different energies as listed in Table 1, that form the basis of the determination of the energy dependencies of the model parameters in Sect. 5. The energy dependencies of both proton-proton and proton-antiproton elastic scattering in the $\mathrm{TeV}$ energy range are determined by a set of 10 physical parameters only, as listed in Table 2. As a next step for establishing the reliability of this $s$-dependence of the model parameters, we have performed also the so called validation or sanity tests in Sect. 6: we have cross-checked that the obtained trends reproduce in a statistically acceptable manner each of the measured data also those, that were not utilized so far to establish the $s$-dependencies of the ReBB model parameters. After establishing that the excitation function of the ReBB model reproduces the measured data, we predict the experimentally not yet available large- $t$ differential cross-section of $p p$ collisions at $\sqrt{s}=0.9,4,5$ and $8 \mathrm{TeV}$ and we present the extrapolations of the $p p$ differential cross-sections measured at the LHC energies of 2.76 and $7.0 \mathrm{TeV}$ to the Tevatron energy of $1.96 \mathrm{TeV}$. Vice versa, we also extrapolate the $p \bar{p}$ differential cross-sections from the SPS and Tevatron energies of 0.546 and $1.96 \mathrm{TeV}$ to the LHC energies of 2.76 and $7.0 \mathrm{TeV}$ in Sect. 7. These results are discussed in detail and put into context in Sect. 8. We summarize the results and conclude in Sect. 9.

This work is closed with four Appendices. For the sake of completeness, the unitary, real part extended BialasBzdak model of Ref. [36] is summarized in Appendix A. In Appendix B we derive and detail the relations between the opacity parameter $\alpha$ of the ReBB model and the realto-imaginary ratio $\rho_{0}$. The main properties of Odderon and Pomeron exchange including the corresponding differential and total cross-sections in the $\mathrm{TeV}$ energy range are summarized in Appendix C. Two small theorems are also given here: Theorem I indicates that if the differential cross-sections of elastic $p p$ and $p \bar{p}$ collisions are not the same in the $\mathrm{TeV}$ energy range, then the crossing-odd component of the elastic amplitude (Odderon) cannot vanish, while Theorem II proves that in the framework of the ReBB model, this is indeed due to the difference between the opacity parameters $\alpha(s)$ for $p p$ and $p \bar{p}$ collisions, linking also mathematically the difference 
Table 1 The values of the fitted ReBB model parameters to $p p$ and $p \bar{p}$ data from SPS to LHC energies. The errors and the values are rounded up to three valuable decimal digits. For 7 $\mathrm{TeV}$, the parameter error values shown in parenthesis do not include the contribution from the parameter correlations, i.e., are less than the MINOS errors

\begin{tabular}{lllll}
\hline$\sqrt{s}(\mathrm{TeV})$ & $0.546(p \bar{p})$ & $1.960(p \bar{p})$ & $2.760(p p)$ & $7.000(p p)$ \\
\hline$|t|\left(\mathrm{GeV}^{2}\right)$ & $(0.375,1.210)$ & $(0.380,1.200)$ & $(0.372,0.741)$ & $(0.377,1.205)$ \\
$\chi^{2} / N D F$ & $44.49 / 33$ & $8.22 / 9$ & $17.32 / 16$ & $80.29 / 52$ \\
$\mathrm{CL}(\%)$ & 8.74 & 51.12 & 36.52 & 0.713 \\
$R_{q}(\mathrm{fm})$ & $0.349 \pm 0.003$ & $0.396 \pm 0.006$ & $0.419 \pm 0.011$ & $0.438 \pm 0.005( \pm 0.001)$ \\
$R_{d}(\mathrm{fm})$ & $0.825 \pm 0.004$ & $0.869 \pm 0.012$ & $0.877 \pm 0.014$ & $0.920 \pm 0.009( \pm 0.002)$ \\
$R_{q d}(\mathrm{fm})$ & $0.284 \pm 0.010$ & $0.294 \pm 0.029$ & $0.197 \pm 0.084$ & $0.333 \pm 0.026( \pm 0.002)$ \\
$\alpha$ & $0.117 \pm 0.002$ & $0.163 \pm 0.005$ & $0.126 \pm 0.006$ & $0.125 \pm 0.002( \pm 0.001)$ \\
$\epsilon_{b 1}$ & - & - & $-0.094 \pm 0.946$ & $0.001 \pm 0.003$ \\
$\epsilon_{c 1}$ & $-0.398 \pm 0.911$ & $-0.013 \pm 0.834$ & $0.059 \pm 0.985$ & $-0.091 \pm 0.866$ \\
$\epsilon_{c 2}$ & $-0.090 \pm 0.416$ & - & - & - \\
\hline
\end{tabular}

Table 2 Summary of the parameter values which determine the energy dependence by fitting a linear logarithmic model according to Eq. (10). The values of the parameters are rounded up to three valuable decimal digits. For $R_{q}, R_{d}$ and $R_{q d}$, the values of the parameters $p_{0}$ and $p_{1}$ are given in units of femtometers (fm). For the parameters $\alpha(p p)$ and $\alpha(p \bar{p})$, the parameters $p_{0}$ and $p_{1}$ are dimensionless

\begin{tabular}{llllll}
\hline Parameter & $R_{q}(f m)$ & $R_{d}(f m)$ & $R_{q d}(f m)$ & $\alpha(p p)$ & $\alpha(p \bar{p})$ \\
\hline$\chi^{2} / N D F$ & $1.596 / 2$ & $0.469 / 2$ & $2.239 / 2$ & $0.760 / 2$ & $1.212 / 2$ \\
CL $(\%)$ & 45.03 & 79.10 & 32.65 & 0.68 & 54.54 \\
$p_{0}$ & $0.131 \pm 0.010$ & $0.590 \pm 0.015$ & $0.158 \pm 0.035$ & $0.167 \pm 0.060$ & $-0.103 \pm 0.027$ \\
$p_{1}$ & $0.017 \pm 0.001$ & $0.019 \pm 0.001$ & $0.010 \pm 0.002$ & $-0.003 \pm 0.003$ & $0.018 \pm 0.002$ \\
\hline
\end{tabular}

in the dip-filling property of the differential cross-sections of elastic scattering to the measurement of $\rho$ at the $t=0$ within the ReBB model. The non-linear corrections to the linear in $\ln (s)$ excitation functions are also determined with the help of ISR $p p$ data at $\sqrt{s}=23.5 \mathrm{GeV}$ energy. These results are discussed in Appendix D, and found to have negligible effects on our results presented in the main body of the manuscript, corresponding to the $\mathrm{TeV}$ energy range.

\section{Formalism}

The elastic amplitude $T(s, t)$ (where $s$ is the squared central mass energy, and $t$ is the squared four-momentum transfer) is defined in Ref. [36] by Eq. (6), Eq. (9) and Eq. (29), furthermore summarized also in Appendix A. The experimentally measurable physical quantities, i.e. the elastic differential cross section, the total, elastic and inelastic cross sections and the ratio $\rho_{0}$ are defined, correspondingly, as:

$$
\begin{aligned}
\frac{\mathrm{d} \sigma}{\mathrm{d} t}(s, t) & =\frac{1}{4 \pi}|T(s, t)|^{2}, \\
\sigma_{t o t}(s) & =2 \operatorname{Im} T(s, t=0), \\
\sigma_{e l}(s) & =\int d t \frac{d \sigma}{d t}(s, t), \\
\sigma_{i n}(s) & =\sigma_{t o t}(s)-\sigma_{e l}(s)
\end{aligned}
$$

and
$\rho_{0}(s)=\frac{\operatorname{Re} T(s, t=0)}{\operatorname{Im} T(s, t=0)}$.

The earlier results show that the ReBB model gives statistically acceptable, good quality fits with $\mathrm{CL} \geq 0.1 \%$ to the $p p$ differential cross section data at the ISR energies of 23.5 and $62.5 \mathrm{GeV}$ as well as at the LHC energy of $7 \mathrm{TeV}$, in the $-t \geq 0.377 \mathrm{GeV}^{2}$ kinematic region [36]. Continuing that study, in this work we apply exactly the same formalism, without any change, to the description of the differential cross-sections of proton-antiproton $(p \bar{p})$ scattering.

This allows us to search for Odderon effects by comparing the $p p$ and $p \bar{p}$ differential cross sections at same energies and squared momentum transfer. Any significant difference between the $p p$ and $p \bar{p}$ processes at the same energy at the $\mathrm{TeV}$ scale provides an evidence for the Odderon exchange. In order to make this manuscript as self-contained and complete as reasonably possible, we have provided a derivation of this well-known property, in the form of Theorem I of Appendix C.

\section{Fitting method}

Compered to the earlier ReBB study [36], in order to more precisely estimate the significance of a possible Odderon effect, here we use a more advanced form of $\chi^{2}$ definition 
which relies on a method developed by the PHENIX Collaboration and described in detail in Appendix A of Ref. [46]. This method is based on the diagonalization of the covariance matrix, if the experimental errors can be separated to the following types of uncertainties:

- Type A errors which are point-to-point fluctuating (uncorrelated) systematic and statistical errors;

- Type B errors which are point-to-point varying but correlated systematic uncertainties, for which the point-topoint correlation is $100 \%$;

- Type C systematic errors which are point-independent, overall systematic uncertainties, that scale all the data points up and down by exactly the same, point-to-point independent factor.

In what follows we index these errors with the index of the data point as well as with subscripts $a, b$ and $c$, respectively.

In the course of the minimization of the ReBB model we use the following $\chi^{2}$ function:

$$
\begin{aligned}
\chi^{2}= & \left(\sum_{j=1}^{M}\left(\sum_{i=1}^{n_{j}} \frac{\left(d_{i j}+\epsilon_{b j} \widetilde{\sigma}_{b i j}+\epsilon_{c j} d_{i j} \sigma_{c j}-t h_{i j}\right)^{2}}{\widetilde{\sigma}_{i j}^{2}}\right)\right. \\
& \left.+\epsilon_{b j}^{2}+\epsilon_{c j}^{2}\right)+\left(\frac{d_{\sigma_{t o t}}-t h_{\sigma_{t o t}}}{\delta \sigma_{t o t}}\right)^{2}+\left(\frac{d_{\rho_{0}}-t h_{\rho_{0}}}{\delta \rho_{0}}\right)^{2} .
\end{aligned}
$$

This definition includes type A, point-to-point uncorrelated errors, type B point-to-point dependent but correlated errors and type $\mathrm{C}$, point independent correlated errors. Furthermore, not only vertical, but the frequently neglected horizontal errors are included too. Let us detail below the notation of this $\chi^{2}$ definition, step by step:

- $M$ is the number of sub-datasets, corresponding to several, separately measured ranges of $t$, indexed with subscript $j$, at a given energy $\sqrt{s}$. Thus $\sum_{j=1}^{M} n_{j}$ gives the number of fitted data points at a given center of mass energy $\sqrt{s}$;

- $d_{i j}$ is the $i$ th measured differential cross section data point in sub-dataset $j$ and $t h_{i j}$ is the corresponding theoretical value calculated from the ReBB model;

- $\tilde{\sigma}_{i j}$ is the type A, point-to-point fluctuating uncertainty of the data point $i$ in sub-dataset $j$, scaled by a multiplicative factor such that the fractional uncertainty is unchanged under multiplication by a point-to-point varying factor:

$$
\tilde{\sigma}_{i j}^{2}=\tilde{\sigma}_{a i j}\left(\frac{d_{i j}+\epsilon_{b j} \tilde{\sigma}_{b i j}+\epsilon_{c j} d_{i j} \sigma_{c j}}{d_{i j}}\right)
$$

where the terms

$$
\tilde{\sigma}_{k i j}=\sqrt{\sigma_{k i j}^{2}+\left(d_{i j}^{\prime} \delta_{k} t_{i j}\right)^{2}}, \quad k \in\{a, b\},
$$

include also the A and B type horizontal errors on $t$ following the propagation of the horizontal error to the $\chi^{2}$ as utilized by the so-called effective variance method of the CERN data analysis programme ROOT; $d_{i j}^{\prime}$ denotes the numerical derivative in point $t_{i j}$ with errors of type $k \in\{a, b\}$, denoted as $\delta_{k} t_{i j}$. The numerical derivative is calculated as

$$
d^{\prime}\left(t_{i j}\right)=\frac{d_{(i+1) j}-d_{i j}}{t_{(i+1) j}-t_{i j}}
$$

- The correlation coefficients for type B and C errors are denoted by $\epsilon_{b}$ and $\epsilon_{c}$, respectively. These numbers are free parameters to be fitted to the data, their best values are typically in the interval $(-1,1)$;

- The last two terms in Eq. (6) are to fit also the measured total cross-section and ratio $\rho_{0}$ values along the differential cross section data points; $d_{\sigma_{\text {tot }}}$ and $d_{\rho_{0}}$ denote the measured total cross section and ratio $\rho_{0}$ values, $\delta \sigma_{\text {tot }}$ and $\delta \rho_{0}$ are their full errors, $\sigma_{\text {tot,th }}$ and $\rho_{0 \text {,th }}$ are their theoretical value calculated from the ReBB model;

This scheme has been validated by evaluating the $\chi^{2}$ from a full covariance matrix fit and from the PHENIX method of diagonalizing the covariance matrix of the differential cross-section of elastic $p p$ scattering measured by TOTEM at $\sqrt{s}=13 \mathrm{TeV}$ [6], using the Lévy expansion method of Ref. [12]. The fit with the full covariance matrix results in the same minimum within one standard deviation of the fit parameters [32], hence in the same significance, as the fit with the PHENIX method. Based on this validation, we apply the PHENIX method in the data analysis described in this manuscript.

Let us note also that in case of the $\sqrt{s}=7 \mathrm{TeV}$ TOTEM data set, analysed below, the B type systematic errors, that shift all the data points together up or down with a $t$ dependent value are measured to be asymmetric [47]. This effect is handled by using the up or down type B errors depending on the sign of the correlation coefficient $\epsilon_{b}$ : for positive or negative sign of $\epsilon_{b}$, we utilized the type B errors upwards, or downwards, respectively. Note that the type A errors, that enter the denominator of the $\chi^{2}$ definition of Eq. (6), are symmetric even in the case of this $\sqrt{s}=7 \mathrm{TeV}$ $p p$ dataset. The $\chi^{2}$ distribution assumes symmetric type $\mathrm{A}$ errors that enter the denominators of the $\chi^{2}$ definition. Thus, even in this case of asymmetric type B errors, that enter the numerators of Eq. (6) at $\sqrt{s}=7 \mathrm{TeV}$, the $\chi^{2}$ distribution can be utilized to estimate the significances and confidence levels of the best fits. 


\section{Fit results}

The ReBB model was fitted to the proton-proton differential cross section data measured by the TOTEM Collaboration at $\sqrt{s}=2.76,7.0$ and $13 \mathrm{TeV}$, based on Refs. $[6,9,47]$ as well as to differential cross section data of elastic protonantiproton scattering measured at $\sqrt{s}=0.546$ and $1.96 \mathrm{TeV}$ in Refs. [45,48,49], respectively.

Similarly to earlier studies of Refs. [34-37,40], the model parameters $A_{q q}=1$ and $\lambda=\frac{1}{2}$ were kept at constant values throughout the fitting procedure. Here $A_{q q}$ corresponds to a normalization constant and $\lambda$ describes the mass ratio of constituent quarks to diquarks in the $p=(q, d)$ version of the real extended Bialas-Bzak model of Ref. [36]. Thus the number of free parameters of this model, for a fixed $s$ and specific collision type is reduced to four: $R_{q d}, R_{q}, R_{d}$ and $\alpha$. It is natural to expect that $R_{q}(s), R_{d}(s)$ and $R_{q d}(s)$ are the same functions of $s$, both for $p p$ and $p \bar{p}$ collisions, as the distribution of partons inside protons at a given energy is expected to be the same as that of anti-partons inside antiprotons. In this section, this is however not assumed but tested and the parameters of the ReBB model are determined at four different colliding energies in the $\mathrm{TeV}$ region, using $p p$ data sets at $\sqrt{s}=2.76$ and $7 \mathrm{TeV}$, and $p \bar{p}$ datasets at $\sqrt{s}=0.546$ and $1.96 \mathrm{TeV}$. These fits were performed in the diffractive interference or dip and bump region, with datapoints before the diffractive minimum and after the maximum as well, in each case the limited range is not greater than $0.372 \leq-t \leq$ $1.2 \mathrm{GeV}^{2}$. In this kinematic range, the ReBB model provided a data description with a statistically acceptable fit quality, with confidence levels $\mathrm{CL} \geq 0.1 \%$ in each case.

In this manuscript, our aim is to extrapolate the differential cross-section of elastic $p p$ and $p \bar{p}$ collisions to exactly the same energies, in order to conclude in a model dependent way about the significance of a crossing-odd or Odderon effect in these data. For this purpose, a model that can be used to study the excitation function of the $p p$ and $p \bar{p}$ differential crosssections in the $0.5 \leq \sqrt{s} \leq 7 \mathrm{TeV}$ domain is sufficient. The results of such kind of statistically acceptable quality fits are summarized in Table 1 and detailed below. Other data sets, that do not have sufficient amount of data in this interference region were utilized for cross-checks only, to test the extracted energy dependencies of the model parameters as detailed in Sect. 6. Additionally, we also describe the current status of our fits to describe the differential cross-section at $\sqrt{s}=13 \mathrm{TeV}$ at the end of this section.

We thus describe three fits to $p p$ differential cross section data sets at $\sqrt{s}=2.76,7$ and $13 \mathrm{TeV}$ as well as two fits to $p \bar{p}$ differential cross section datasets at $\sqrt{s}=0.546$ and $1.96 \mathrm{TeV}$, respectively. Our fit results are graphically shown in Figs. 1, 2, 3, 4 and 5.

The minimization of the $\chi^{2}$ defined by Eq. (6) was done with Minuit and the parameter errors were estimated by using

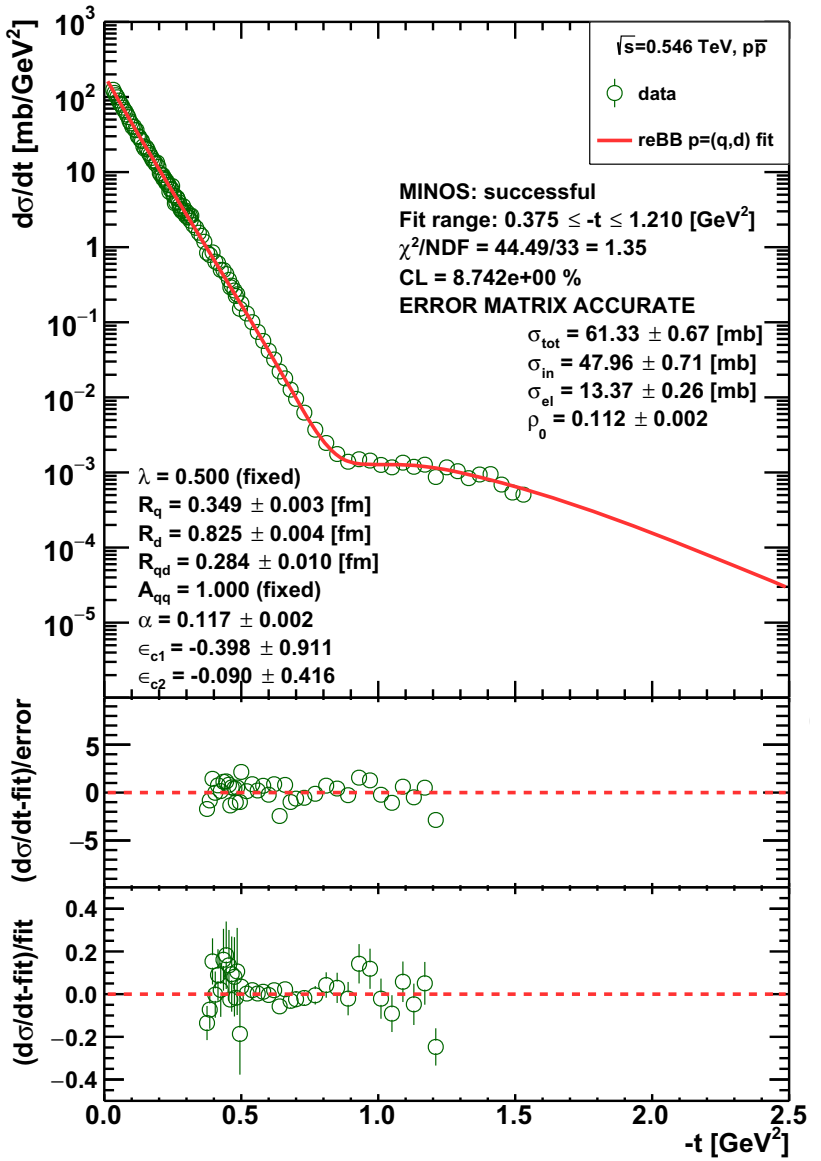

Fig. 1 The fit of the ReBB model to the $p \bar{p}$ SPS $\sqrt{s}=0.546 \mathrm{TeV}$ data $[48,49]$ in the range of $0.37 \leq-t \leq 1.2 \mathrm{GeV}^{2}$. The fit includes the published errors, that are statistical (type A) and the normalization (type C) uncertainties, as well as the experimental value of the total cross section with its full error according to Eq. (6). The fitted parameters are shown in the left bottom corner and their values are rounded up to three decimal digits. The fit quality parameters and the values of the total, inelastic and elastic cross-sections as well as the value of the $\rho_{0}$ parameter are summarized in the top right part of the plot

the MINOS algorithm which takes into account both parameter correlations and non-linearities. We accept the fit as a successful representation of the fitted data under the condition that the fit status is converged, the error matrix is accurate and the confidence level of the fit, $\mathrm{CL}$ is $\geq 0.1 \%$, as indicated on Figs. 1, 2, 3 and 4. As these criteria are not satisfied on Fig. 5, the parameters of this fit were not taken into account when determining the excitation functions or the energy dependence of the physical fit parameters in the few $\mathrm{TeV}$ energy range.

Let us now discuss each fit in a bit more detail.

The $\mathrm{S} p \bar{p} \mathrm{~S}$ differential cross section data on elastic $p \bar{p}$ collisions $[48,49]$ were measured in the squared momentum transfer range of $0.03 \leq|t| \leq 1.53 \mathrm{GeV}^{2}$ which in the fitted range has been subdivided into two sub-ranges with different 


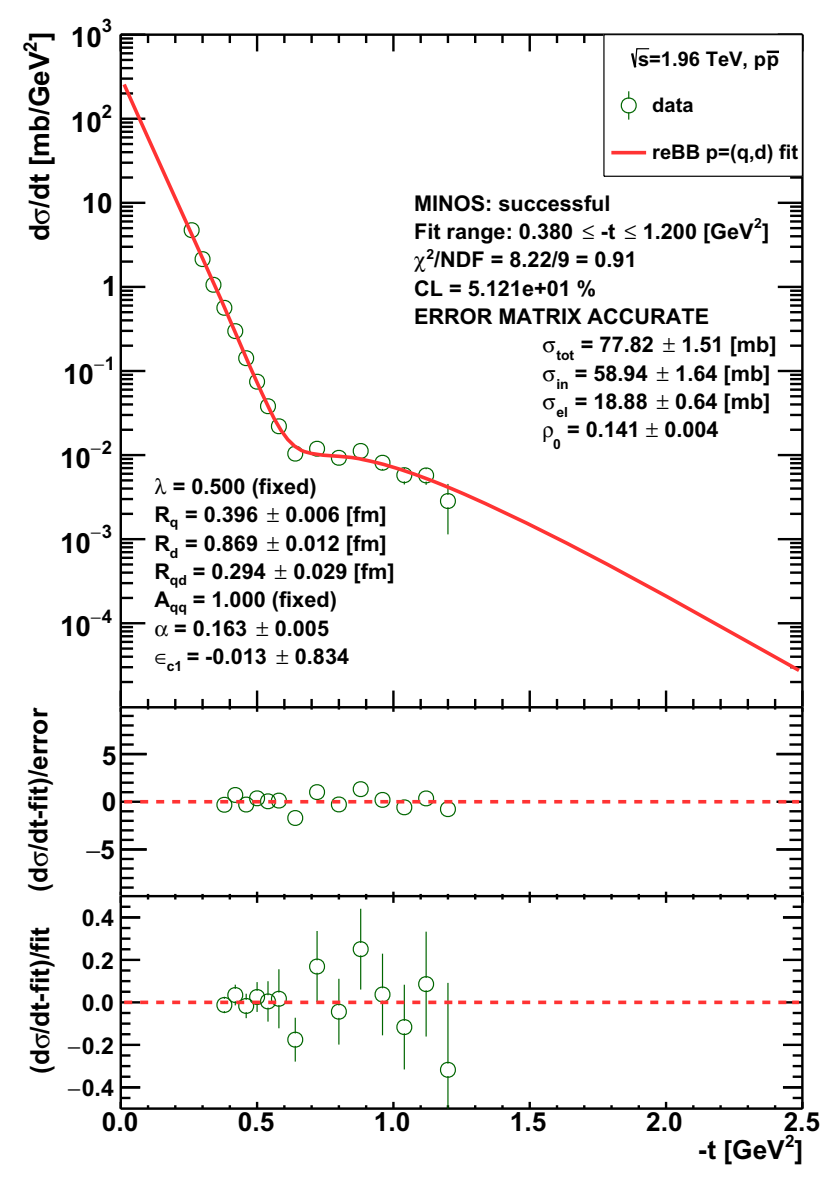

Fig. 2 The fit of the ReBB model to the $p \bar{p}$ D0 $\sqrt{s}=1.96 \mathrm{TeV}$ data [45] in the range of $0.37 \leq-t \leq 1.2 \mathrm{GeV}^{2}$. The fit includes the $t$-dependent statistical and systematic uncertainties added together quadratically and treated as type A errors as well as the normalization (type C) uncertainty according to Eq. (6). The values of the total cross section and parameter $\rho_{0}$ used in the fit are the predicted values from the COMPETE Collaboration [51]. Otherwise, same as Fig. 1

normalization uncertainties (type $\mathrm{C}$ errors): for $0.37 \leq|t| \leq$ $0.495 \mathrm{GeV}^{2} \sigma_{c}=0.03$ and for $0.46 \leq|t| \leq 1.2 \mathrm{GeV}^{2}$ $\sigma_{c}=0.1$. In case of this data set, the vertical type A errors $\sigma_{a i}$ are available but the horizontal type A errors $\left(\delta_{a} t_{i}\right)$ and the type B errors either vertical $\left(\sigma_{b i}\right)$ or horizontal $\left(\delta_{b} t_{i}\right)$ were not published. The measured total cross section with its total uncertainty is $\sigma_{\text {tot }}=61.26 \pm 0.93 \mathrm{mb}$ [50] while the $\rho_{0}=$ $0.135 \pm 0.015$ value was measured at the slightly different energy of $\sqrt{s}=0.541 \mathrm{GeV}$. The total, elastic and inelastic cross sections and the parameter $\rho_{0}$ are calculated according to Eqs. (2)-(5). The fit is summarized in Fig. 1. The fit quality is satisfactory, $\mathrm{CL}=8.74 \%$. Compared to the available data in the literature [50] $\left(\sigma_{i n}=48.39 \pm 1.01 \mathrm{mb}\right.$ and $\sigma_{e l}=12.87 \pm$ $0.3 \mathrm{mb}$ ) the model reproduces the experimental values of the forward measurables within one $\sigma$, thus these fit parameters represent the data in a statistically acceptable manner.

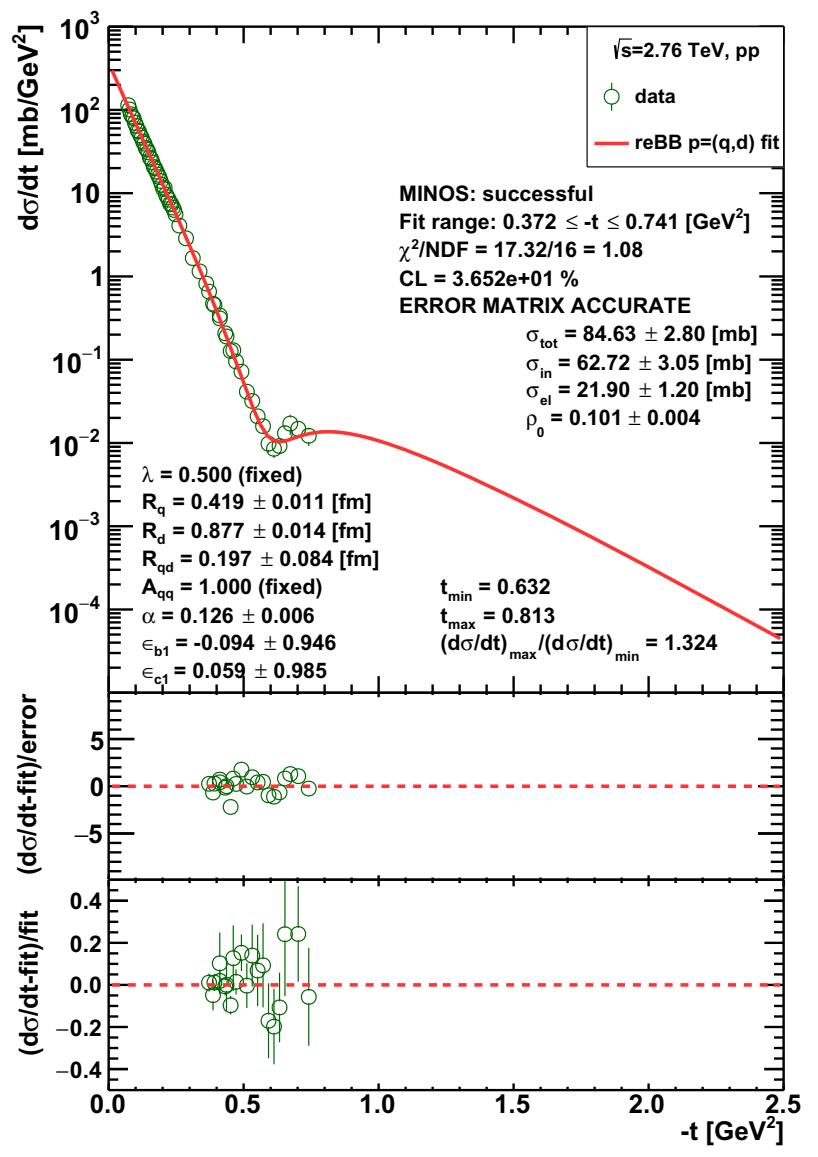

Fig. 3 The fit of the ReBB model to the $p p$ TOTEM $\sqrt{s}=2.76 \mathrm{TeV}$ data in the range of $0.37 \leq-t \leq 0.74 \mathrm{GeV}^{2}$ [9]. The fit includes the $t$-dependent statistical (type A) and systematic (type B) uncertainties, the normalization (type C) uncertainty and the experimental value of the total cross section with its full error according to Eq. (6). Otherwise, same as Fig. 1

The elastic $p \bar{p}$ differential cross section data is available at $\sqrt{s}=1.96 \mathrm{TeV}$ in the range of $0.26 \leq|t| \leq 1.20 \mathrm{GeV}^{2}$, as published by the D0 Collaboration in Ref. [45], with a type $\mathrm{C}$ normalization uncertainty of $\sigma_{c}=0.144$. For this data set, the vertical type $\mathrm{A}$ and type $\mathrm{B}$ errors were not published separately. Actually, the quadratically added statistical and systematic uncertainties were published, and as the statistical errors are point to-point fluctuating, type A errors, in our analysis the combined $t$ dependent D0 errors were handled as type A, combined statistical and systematic errors. Horizontal type A and type B errors were not published in Ref. [45]. At this energy, we do not find published experimental $\sigma_{\text {tot }}$ and $\rho_{0}$ values. The values of the total cross section and parameter $\rho_{0}$ at this energy, that we utilized in the fitting procedure, are the predicted values from the COMPETE Collaboration [51]: $\sigma_{\text {tot }}=78.27 \pm 1.93 \mathrm{mb}$ and $\rho_{0}=0.145 \pm 0.006$. The quality of the corresponding fit, shown in Fig. 2, is satisfactory, $\mathrm{CL}=$ $51.12 \%$, and the COMPETE values of forward measurables are reproduced within one standard deviation. We conclude 


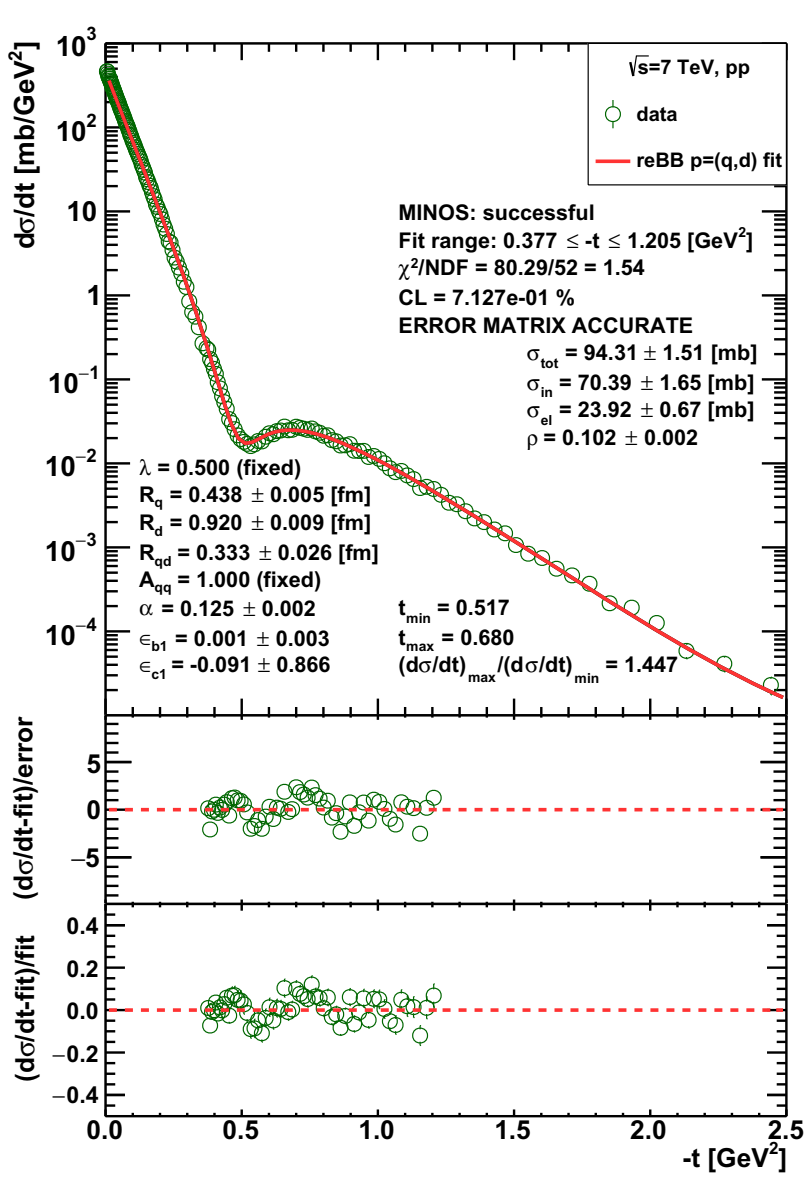

Fig. 4 The fit of the ReBB model to the $p p$ TOTEM $\sqrt{s}=7 \mathrm{TeV}$ data in the range of $0.37 \leq-t \leq 1.2 \mathrm{GeV}^{2}$ [47]. The fit includes the $t$-dependent statistical (type A) and systematic (type B) uncertainties, the normalization (type $\mathrm{C}$ ) uncertainty and the experimental values of the total cross section and parameter $\rho_{0}$ with their full error according to Eq. (6). Otherwise, same as Fig. 1

that the corresponding ReBB model parameters represent the data in a statistically acceptable manner.

Based on the successful description of these two $p \bar{p}$ datasets at $\sqrt{s}=0.546$ and $1.96 \mathrm{TeV}$, we find that the form of the ReBB model as specified for $p p$ collisions in Ref. [36] is able, without any modifications, to describe the differential cross-section of elastic $p \bar{p}$ collisions in the TeV energy range. Let us now discuss the new fits of the same model to elastic $p p$ collisions in the TeV energy range.

At $\sqrt{s}=2.76 \mathrm{TeV}$, the differential cross section data of elastic $p p$ collisions was measured in the $t$ range of $0.072 \leq-t \leq 0.74 \mathrm{GeV}^{2}$ by the TOTEM Collaboration [9]. Actually, this measurement was performed in two subranges: $0.072 \leq|t| \leq 0.462 \mathrm{GeV}^{2}$ and $0.372 \leq|t| \leq 0.74$ $\mathrm{GeV}^{2}$. Both ranges had the same normalization uncertainty of $\sigma_{c}=0.06$. During the fit the $t$-dependent vertical statistical (type A) and vertical systematic (type B) errors, the normalization (type $\mathrm{C}$ ) errors and the experimental value of the total

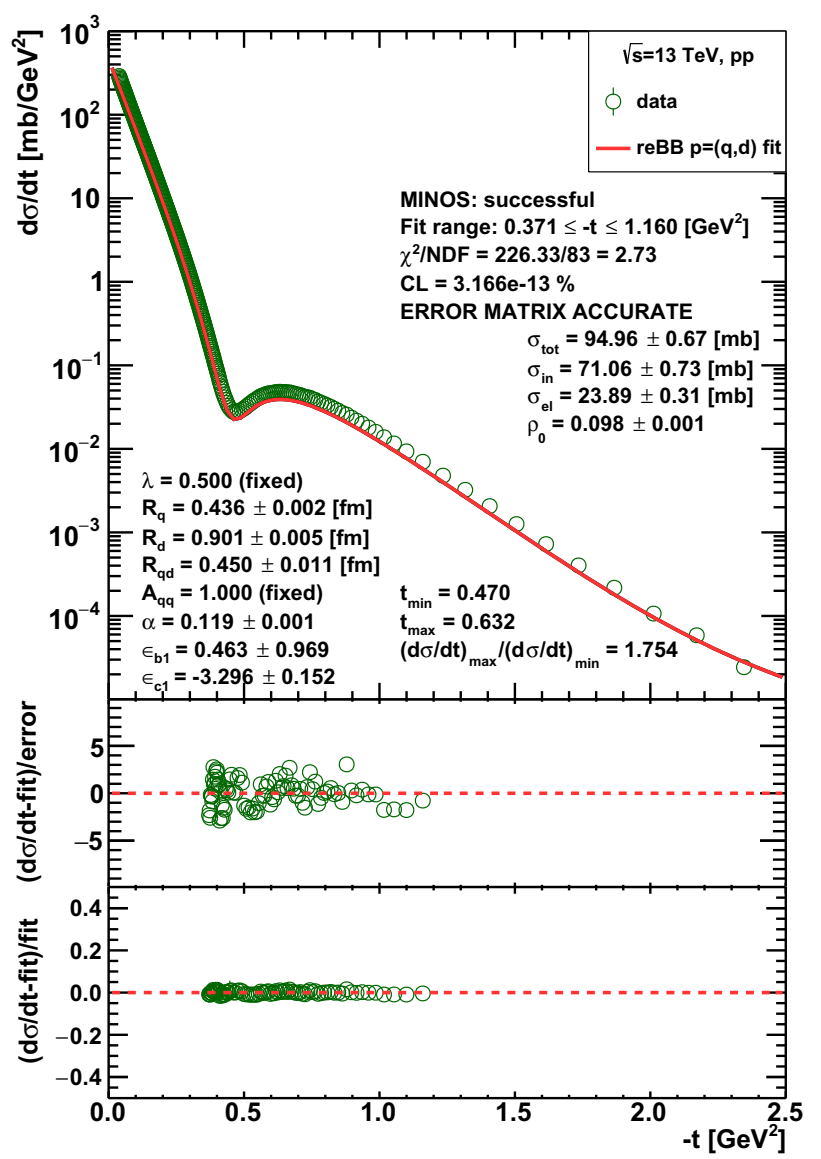

Fig. 5 The fit of the ReBB model to the $p p$ TOTEM $\sqrt{s}=13 \mathrm{TeV}$ data in the range of $0.37 \leq-t \leq 1.2 \mathrm{GeV}^{2}$ [8]. The fit includes the $t$-dependent statistical (type A) and systematic (type B) uncertainties, the normalization (type $\mathrm{C}$ ) uncertainty and the experimental values of the total cross section and parameter $\rho_{0}$ with their full error according to Eq. (6). The fit parameters do not represent the data in a statistically acceptable manner, given that $\mathrm{CL} \ll 0.1 \%$. Otherwise, same as Fig. 1

cross section with its total uncertainty $\left(\sigma_{\text {tot }}=84.7 \pm 3.3 \mathrm{mb}\right.$ [6]) were taken into account. Horizontal type A and type B errors are not published at this energy. The fit quality of the ReBB model is demonstrated on Fig. 3: the fit is satisfactory, with $\mathrm{CL}=36.52 \%$. The experimental values of the forward measurables $\left(\sigma_{i n}=62.8 \pm 2.9 \mathrm{mb}, \sigma_{e l}=21.8 \pm 1.4\right.$ $\mathrm{mb}[6,52])$ are reproduced within one standard deviations. Experimental data is not yet available for parameter $\rho_{0}$, however the value for $\rho_{0}$, calculated from the fitted ReBB model, is within the total error band of the COMPETE prediction [51]. We thus conclude that the corresponding ReBB model parameters represent the $p p$ data at $\sqrt{s}=2.76 \mathrm{TeV}$ in a statistically acceptable manner.

At $\sqrt{s}=7 \mathrm{TeV}$, the $p p$ differential cross section data was published by the TOTEM Collaboration [47], measured in the range of $0.005 \leq|t| \leq 2.443 \mathrm{GeV}^{2}$. The measurement 
was performed in two subranges: $0.005 \leq|t| \leq 0.371 \mathrm{GeV}^{2}$ and $0.377 \leq|t| \leq 2.443 \mathrm{GeV}^{2}$. Both ranges had the same normalization uncertainty of $\sigma_{c}=0.042$. The fit includes only the second subrange with the $t$-dependent (both vertical and horizontal) statistical (type A) and systematic (type B) errors (both vertical and horizontal ones), the normalization (type $\mathrm{C}$ ) error and the experimental values of the total cross section and the parameter $\rho_{0}$ with their total uncertainties $\left(\sigma_{\mathrm{tot}}=98.0 \pm 2.5 \mathrm{mb}\right.$ and $\rho_{0}=0.145 \pm 0.091$ [53]). The quality of the corresponding fit, shown in Fig. 4, is statistically acceptable with a $\mathrm{CL}=0.71 \%$. The experimental values of the forward measurables $\left(\sigma_{i n}=72.9 \pm 1.5 \mathrm{mb}\right.$, $\sigma_{e l}=25.1 \pm 1.1 \mathrm{mb}$ [53]) are reproduced by the fitted ReBB model within one sigma (the experimental and calculated values overlap within their errors). We thus conclude that the corresponding ReBB model parameters represent these $p p$ data at $\sqrt{s}=7.0 \mathrm{TeV}$ in a statistically acceptable manner, in the fitted range of $0.377 \leq|t| \leq 1.205 \mathrm{GeV}^{2}$, before and after the diffractive minimum.

At $\sqrt{s}=8 \mathrm{TeV}$, the TOTEM collaboration did not yet publish the final differential cross-section results in the range of the diffractive minimum and maximum. However, preliminary results were presented at conferences [54], and the differential cross-section in the low $-t$ region was published in Ref. [55]. We thus use this dataset for a cross-check only, but the lack of the data in the diffractive minimum prevents us to do a full ReBB model fit. Additional data at very low $-t$, in the Coulomb-Nuclear Interference region is also available from TOTEM at this particular energy [56], however, in the present study we do not discuss the kinematic range, where Coulomb effects may play any role.

At $\sqrt{s}=13 \mathrm{TeV}$, the differential cross section data was measured by the TOTEM collaboration in the range of $0.03 \leq|t| \leq 3.8 \mathrm{GeV}^{2}$ [8] with a normalization (type C) uncertainty of $\sigma_{c}=0.055$. As far as we know, the only statistically acceptable quality fit with $\mathrm{CL} \geq 0.1 \%$ to this dataset so far was obtained by some of us with the help of the modelindependent Lévy series in Ref. [12]. We also note that several new features show up in the soft observables of elastic scattering, with a threshold behaviour around $\sqrt{s}=5-7$ $\mathrm{TeV}$, certainly below $13 \mathrm{TeV}$ [57].

We have cross-checked, if the ReBB model, that works reasonably well from $\sqrt{s}=23.5 \mathrm{GeV}$ to $7 \mathrm{TeV}$, is capable to describe this data set at $\sqrt{s}=13 \mathrm{TeV}$ in statistically acceptable manner, or not? The result was negative, as indicated in Fig. 5. This fit includes the $t$-dependent statistical (type A) and systematic (type B) errors, the normalization (type $\mathrm{C}$ ) error and the experimental values of the total cross section and the parameter $\rho_{0}$ with their total uncertainties $\left(\sigma_{\text {tot }}=110.5 \pm 2.4 \mathrm{mb}\right.$ and $\rho_{0}=0.09 \pm 0.01$ [7]). The quality of the obtained fit (Fig. 5) is not satisfactory, $\mathrm{CL}=3.17 \times 10^{-11} \%$ and neither the experimental values of the cross sections $\left(\sigma_{i n}=79.5 \pm 1.8 \mathrm{mb}, \sigma_{e l}=31.0 \pm 1.7\right.$ $\mathrm{mb}[6]$ ) are reproduced by the fitted ReBB model within one sigma at $13 \mathrm{TeV}$. However, the value of $\rho_{0}$ was described surprisingly well. This TOTEM dataset is very detailed and precise and changes of certain trends in $B(s)$ and the ratio $\sigma_{\text {el }}(s) / \sigma_{\text {tot }}(s)$ are seen experimentally [57]. Theoretically, a new domain of QCD may emerge at high energies, possibly characterised by hollowness or toroidal structure, corresponding to a black ring-like distribution of inelastic scatterings [58-61]. A statistically significant, more than $5 \sigma$ hollowness effect was found at $\sqrt{s}=13 \mathrm{TeV}$ within a modelindependent analysis of the shadow profile at these energies, using the technique of Lévy series [12]. We conclude that the ReBB model needs to be generalized to have a stronger non-exponential feature at low $-t$ to accommodate the new features of the differential cross-section data at $\sqrt{s}=13$ $\mathrm{TeV}$ or larger energies. This work is currently in progress, but goes well beyond the scope of the current manuscript. Most importantly, such a generalization is not necessary for a comparison of the differential cross-sections of elastic $p p$ and $p \bar{p}$ collisions in the few $\mathrm{TeV}$ range, as we have to bridge only a logarithmically small energy difference between the top D0 energy of $\sqrt{s}=1.96 \mathrm{TeV}$ and the lowest TOTEM energy of $\sqrt{s}=2.76 \mathrm{TeV}$.

We thus find, that the Real Extended Bialas - Bzdak model describes effectively and in a statistically acceptable manner the differential cross-sections of elastic $p p$ and $p \bar{p}$ collisions in the few $\mathrm{TeV}$ range of $0.546 \leq \sqrt{s} \leq 7 \mathrm{TeV}$ and in the squared four-momentum transfer range of $0.37 \leq-t \leq$ $1.2 \mathrm{GeV}^{2}$. Its physical fit parameters represent the data and their energy dependence thus can be utilized to determine the excitation function of these model parameters, as detailed in Sect. 5.

The values of the physical fit parameters and their errors obtained from the above discussed physically and statistically acceptable fits are summarized in Table 1, where four datasets are analyzed and four different physical parameters are extracted at four different energies. These sixteen physical parameters form the basis of the determination of the energy dependencies, that are determined to be consistent with affine linear functions of $\ln (s)$. Three scale parameters are within errors the same in elastic $p p$ and $p \bar{p}$ collisions, while the opacity parameters are different for $p p$ and $p \bar{p}$ collisions. Thus the excitation functions, the energy dependence of the differential cross-sections both for $p p$ and $p \bar{p}$ elastic scattering is determined by $5 \times 2=10$ physical parameters in this framework of calculations. These 10 parameters are summarized in Table 2.

We thus conclude, that this real extended Bialas-Bzdak model is good enough to extrapolate the differential crosssection of elastic $p p$ collisions down to $\sqrt{s}=0.546$ and 1.96 $\mathrm{TeV}$, and to extrapolate the same of elastic $p \bar{p}$ collisions up to $\sqrt{s}=2.76$ and $7 \mathrm{TeV}$. We duly note that, in order to evaluate similar observables at $\sqrt{s}=13 \mathrm{TeV}$ or at even 


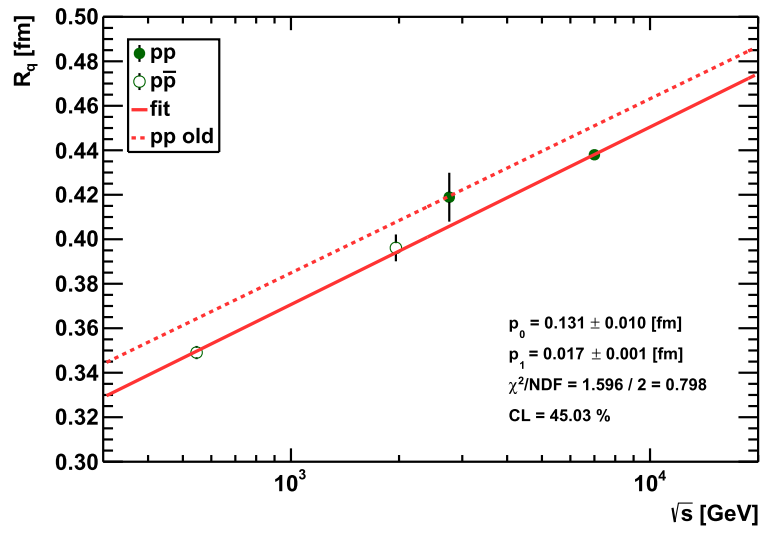

(a) Parameter $R_{q}$

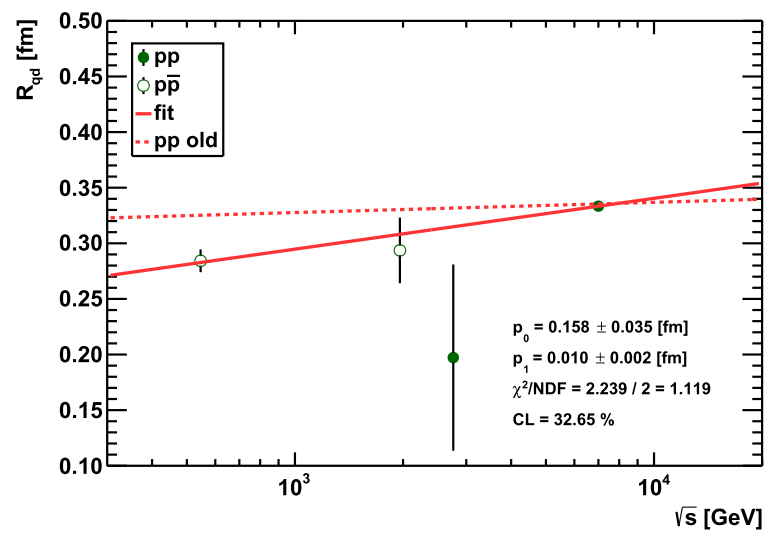

(c) Parameter $R_{q d}$

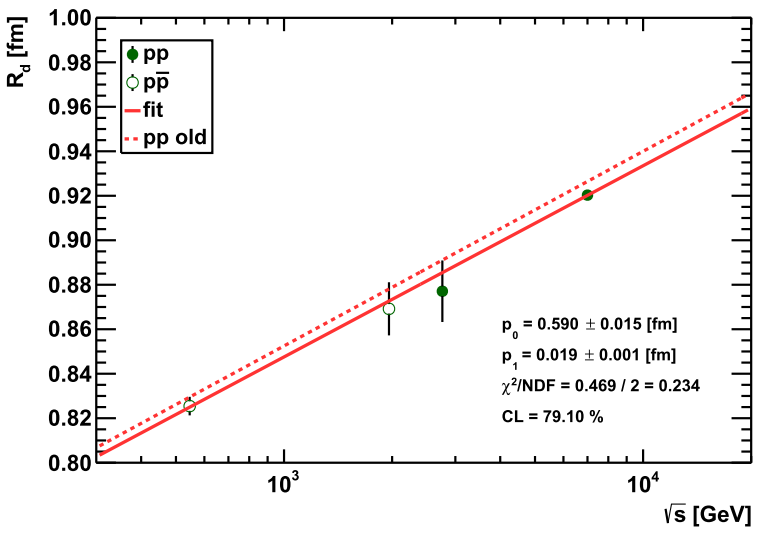

(b) Parameter $R_{d}$

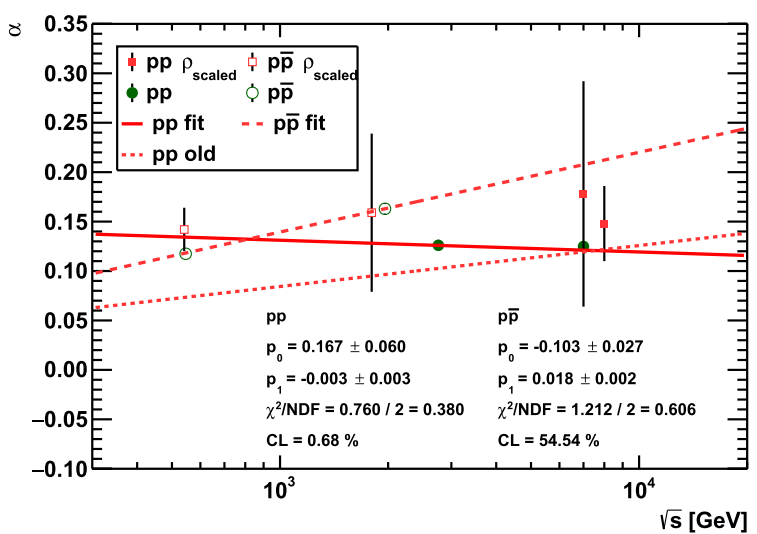

(d) Parameter $\alpha$

Fig. 6 The energy dependence of the parameters of the ReBB model, $R_{q}, R_{d}, R_{q d}$ and $\alpha$, collected in Table 1 and determined by fitting a linear logarithmic model, Eq. (10), to each of them one by one

higher energies in a realistic manner, this model needs to be generalized and further developed.

\section{Excitation functions of the fit parameters}

The values of the physical fit parameters and their errors obtained from the above discussed physically and statistically acceptable fits are summarized in Table 1 . This table contains a list of five different physical parameters. Out of them the three scale parameters called $R_{q}, R_{d}$ and $R_{q d}$ can be determined at four different energies, providing 12 numbers, while the opacity parameters $\alpha^{p p}$ and $\alpha^{p \bar{p}}$ describing $p p$ and $p \bar{p}$ collisions can both be determined at two different energies only, providing additional 4 numbers, all-together 16 physical input parameters. These 16 physical parameters form the basis of the determination of the energy dependencies, that are determined to be consistent with affine linear functions of $\ln (s)$.
Namely, we fitted the $s$-dependence of the model parameters one by one, using the affine linear logarithmic function,

$$
P(s)=p_{0}+p_{1} \cdot \ln \left(s / s_{0}\right), \quad P \in\left\{R_{q}, R_{d}, R_{q d}, \alpha\right\},
$$

where $p_{0}$ and $p_{1}$ are free parameters, $s_{0}$ is fixed at $1 \mathrm{GeV}^{2}$. We obtain good quality fits, with methods and results similar to that of Ref. [36], with confidence levels $C L \gg 0.1 \%$, as detailed in Table 2. Three scale parameters are within errors the same in elastic $p p$ and $p \bar{p}$ collisions, while the opacity parameters are different for $p p$ and $p \bar{p}$ collisions. Thus the excitation functions, the energy dependence of the differential cross-sections both for $p p$ and $p \bar{p}$ elastic scattering is determined by $5 \times 2=10$ physical parameters in the framework of the ReBB model.

The energy dependence of the scale parameters, $R_{q}, R_{d}$, and $R_{q d}$ are graphically shown in Fig. 6a-c. These figures clearly indicate that the energy dependence of the geometrical scale parameters consistent with the same evolution, namely the same linear rise in $\ln (s)$ for both $p p$ and $p \bar{p}$ 
scattering: when we fitted these parameters together, with a linear logarithmic function, we have obtained a statistically acceptable fit in each of these three cases. This result extends and improves the earlier results published in Ref. [36] for elastic $p p$ scattering to the case of both $p p$ and $p \bar{p}$ collisions in a natural manner. For a comparision, these earlier results are also shown with a dotted red line on the panels of Fig. 6 , indicating the improved precision of the current analysis, due to more data points are included in the $\mathrm{TeV}$ energy range.

For the opacity parameter $\alpha$, seen on panel (d) of Fig. 6, the situation is different: the $p p$ and $p \bar{p}$ points are not on the same trend, because the $\alpha$ parameters that characterize the dip in the ReBB model, are obtained with great precision both in the $p p$ and in the $p \bar{p}$ cases. The difference between the excitation functions of $\alpha^{p p}(s)$ and $\alpha^{p \bar{p}}(s)$ corresponds to the qualitative difference between the differential cross-section of elastic $p p$ and $p \bar{p}$ collisions in the few $\mathrm{TeV}$ energy range: the presence of a persistent dip and bump structure in the differential cross-section of elastic $p p$ collisions, and the lack of a similar feature in elastic $p \bar{p}$ collisions. Thus in the case of parameter $\alpha$ we have to consider, that there are only two, rather precisely determined data points in both $p p$ and $p \bar{p}$ collisions from the presented ReBB model studied so far. We can already conclude that they cannot be described by a single line as an affine linear fit with Eq. (10) would fail. Without additional information, we cannot determine the trends and its uncertainties as two points can always connected with a straight line, so an affine linear description of both the two $p p$ and the two $p \bar{p}$ data points would have a vanishing $\chi^{2}$ and an indeterminable confidence level. This problem, however, is solved by utilizing the results of Appendix B on the proportionality between the model parameter $\alpha$ and the experimentally measurable real-to-imaginary ratio $\rho_{0}$. This proportionality is shown graphically in Fig. 7. The constant of proportionality in the few $\mathrm{TeV}$ region is an almost energy independent constant value, $\rho_{0} / \alpha=0.85 \pm 0.01$, well within the errors of the $\rho_{0}$ measurements, in agreement with a theoretically obtained function, showed with a red solid line on Fig. 7 and derived in Appendix B. This proportionality allows one to add new datapoints to the trends of $\alpha(s)$ both for the $p p$ and for the $p \bar{p}$ cases by simply rescaling the measured $\rho_{0}$ values.

We found three additional published experimental data of $\rho_{0}$ for $p \bar{p}$ collisions, $\rho_{0}=0.135 \pm 0.015$ at $\sqrt{s}=0.541$ by the UA4/2 Collaboration in Ref. [62] and $1.8 \mathrm{TeV}$ by the E-710 and the E811 collaborations in Refs. [63,64], respectively. At $\sqrt{s}=1.8 \mathrm{TeV}$, we have utilized the combined value of these E-710 and E811 measurements [64], corresponding to $\rho_{0}(p \bar{p})=0.135 \pm 0.044$. The constancy of these $\rho_{0}(s)$ values in the few $\mathrm{TeV}$ energy range, when converted with the help of Fig. 7 to the opacity parameter $\alpha(p \bar{p})$ of the Bialas-Bzdak model, leads to the lack of diffractive minima hence an Odderon signal in elastic $p \bar{p}$ collisions, leading to

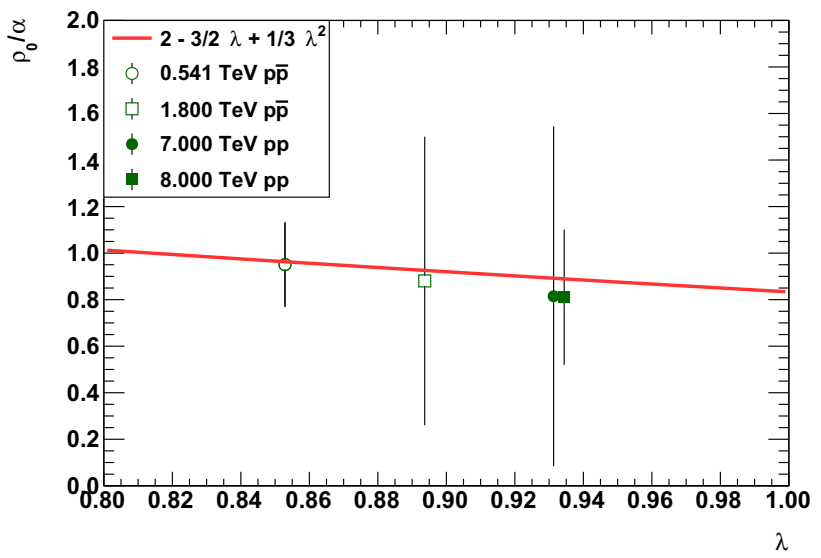

Fig. 7 The dependence of $\rho_{0} / \alpha$ on $\lambda$ in the TeV energy range. The data points are generated numerically by using the trends of the ReBB model parameters, $R_{q}, R_{d}, R_{q d}$, shown in Fig. 6a-c and the experimentally measured ratio $\rho_{0}$ values. The red curve represents the result of the analytical calculation showing a good agreement with the numerical calculations

an $\alpha(p \bar{p}) \approx 0.16 \pm 0.06$ which is within its large errors the same as the $\alpha=0.163 \pm 0.005$ value obtained from the ReBB model fit to D0 data at $\sqrt{s}=1.96 \mathrm{TeV}$, summarized on Fig. 2. Similarly the $\alpha$ parameter extracted from $\rho_{0}$ at $\sqrt{s}=0.541 \mathrm{TeV}$ is $\alpha \approx 0.16 \pm 0.02$ which is within twice the relatively large errors of the $\rho_{0}$ analysis the same as the value of $\alpha(p \bar{p})=0.117 \pm 0.002$ obtained from the analysis of the differential cross-section, shown on Fig. 1. These indicate a slowly rising value for $\alpha(p \bar{p})$ or correspondingly, $\rho_{0}(p \bar{p})$ in the $\mathrm{TeV}$ energy range. The final values of these datapoints together with the corresponding errors are connected with a long-dashed line in Panel (d) of Fig. 6. Table 2 indicates that for $\alpha(p \bar{p})$ the coefficient $p_{1}(p \bar{p})=0.018 \pm 0.002$ is a significantly positive number.

For the opacity coefficient in elastic $p p$ collisions, $\alpha(p p)$ on the other hand an opposite effect is seen, when the $\rho_{0}$ measurements at $\sqrt{s}=7$ and $8 \mathrm{TeV}$ are also taken into account, based on the data of the TOTEM Collaboration published in Refs. [56,65]. As by now it is very well known, these values indicate a nearly constant, actually decreasing trend, and based on the fits of the extracted four data points of $\alpha(p p)$ we find that in the few $\mathrm{TeV}$ energy range, this trend is nearly constant, indicated by the solid red line of panel (d) of Fig. 6. Table 2 indicates that for $\alpha(p p)$ the coefficient of increase with $\ln (s)$ is consistent with zero in this energy range, $p_{1}(p p)=-0.003 \pm 0.003$, which is significantly less from the above quoted positive number for $p_{1}(p \bar{p})=0.018 \pm 0.002$. Thus it is easy to see, that the Odderon signal in this analysis can be an estimated $6-7 \sigma$ effect, as a consequence of the unequality $p_{1}(p p) \neq p_{1}(p \bar{p})$ alone.

In the subsequent sections we first test if the excitation functions, determined with the help of the $p_{0}$ and $p_{1}$ param- 
eters of Table 2 indeed reproduce the data at all the measured energies in the relevant kinematic range, then we proceed carefully to determine the significance of a model dependent Odderon signal. We perform these cross-checks against all kind of available data, including those data that were not utilized in the determination of the trends for example because their acceptance was too limited to determine all the fit parameters of the ReBB model.

\section{Sanity tests}

In this section we show that the determined energy dependence trends are reliable in the kinematic range of $0.546 \leq$ $\sqrt{s} \leq 8 \mathrm{TeV}$ and $0.37 \leq-t \leq 1.2 \mathrm{GeV}^{2}$. For this purpose we performed the so-called sanity tests: we have crosschecked if the trends summarized in Table 2 indeed represent all the available differential cross-section data on both $p p$ and $p \bar{p}$ elastic scattering in the mentioned kinematic range. We used both those data which were and which were not utilized in the determination of the energy dependence trends for example because their acceptance was too limited to determine all the fit parameters of the ReBB model.

To perform these cross-checks, the differential cross sections are fitted with all the four physical parameters of the ReBB model, $\alpha(s), R_{q}(s), R_{d}(s)$ and $R_{q d}(s)$, fixed to their extrapolated value obtained with the help of the results summarized in Table 2, while the correlation coefficients of the type $\mathrm{B}$ and $\mathrm{C}$ errors, or the $\epsilon$ parameters in the $\chi^{2}$ definition of Eq. (6) are fitted to the data as free parameters.

The results for the data at $\sqrt{s}=0.546,0.63,1.8,1.96$, 2.76 and $7 \mathrm{TeV}$ are shown in Figs. 8, 9, 10, 11, 12 and 13. All of these sanity tests resulted in the description of these data with a statistically acceptable confidence level of CL > $0.1 \%$.

As an additional sanity test, we have also cross-checked if this ReBB model describes the $p p$ and $p \bar{p}$ total cross section $\sigma_{\text {tot }}(s)$ and real to imaginary ratio $\rho_{0}(s)$ data in a statistically acceptable manner, or not. These results are presented in Figs. 14 and 15, respectively. As the calculated confidence levels are higher than $0.1 \%$ in all of these cases, we can happily conclude that the energy dependent trends of the ReBB model are really reasonable and reliable in the investigated $0.541 \leq \sqrt{s} \leq 8 \mathrm{TeV}$ energy and in the $0.377 \leq-t \leq 1.2 \mathrm{GeV}^{2}$ squared four-momentum transfer range. Thus this model can be used reliably to extrapolate both the $p p$ and the $p \bar{p}$ differential cross-sections in this limited kinematic range of $(s, t)$, based only on 10 physical model parameters, summarized in Table 2.

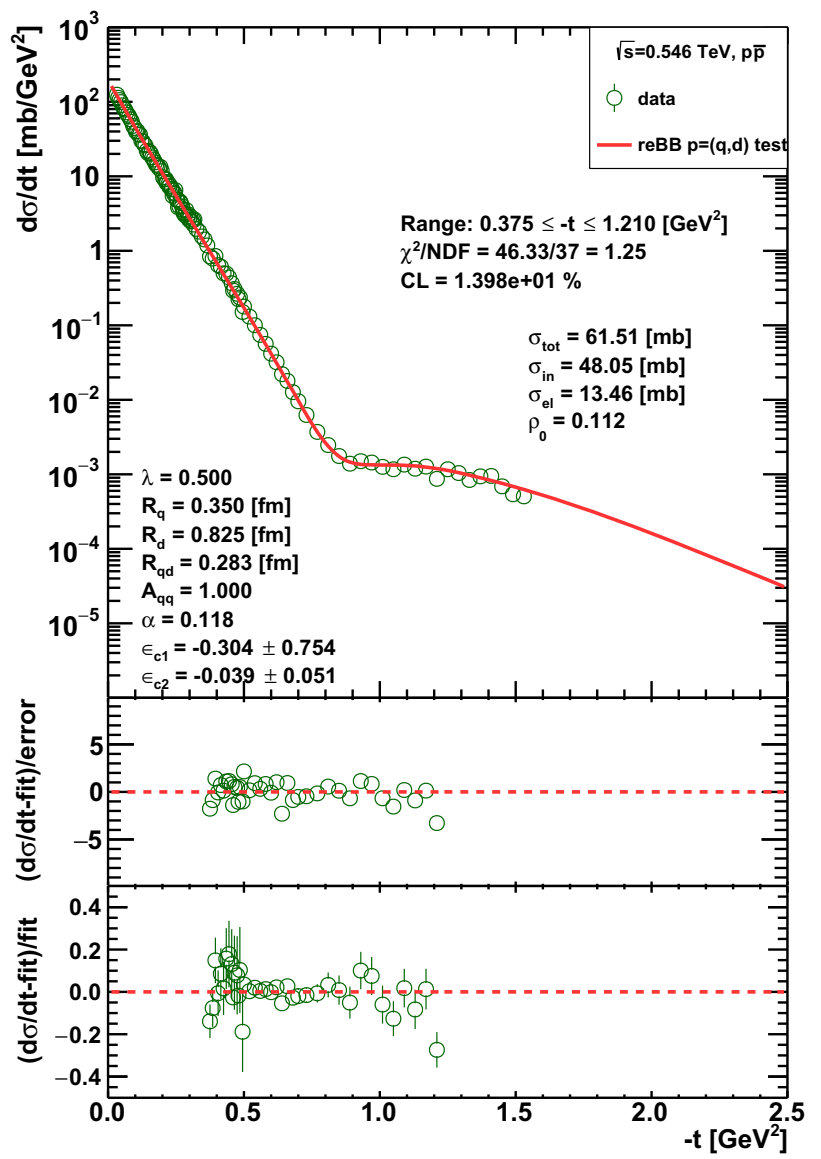

Fig. 8 Result of the sanity test for the $0.546 \mathrm{TeV} p \bar{p}$ elastic differential cross section data $[48,49]$ in the range of $0.37 \leq-t \leq 1.2 \mathrm{GeV}^{2}$. This sanity test was performed as a fit during which the model parameters $R_{q}, R_{d}, R_{q d}$ and $\alpha$ were fixed to their $s$-dependent value based on Table 2, while correlation coefficients $\epsilon$-s in the $\chi^{2}$ definition, Eq. (6), were fitted as free parameters. Thus the physical parameters $R_{q}, R_{d}$, $R_{q d}$ and $\alpha$ are printed on the plot without error bars while the fitted correlation coefficients are given with their errors. The best parameter values are rounded up to three valuable decimal digits

\section{Extrapolations}

According to our findings in Sect. 5 the energy dependences of the scale parameters $R_{q}, R_{d}$ and $R_{q d}$ are identical for $p p$ and $p \bar{p}$ scattering, only the energy dependence of the opacity parameter $\alpha$ differs. The statistically acceptable quality of the fits shown in Fig. 6 and the success of the sanity tests performed in the previous section allow for a reliable extrapolation of the differential cross-sections of elastic $p p$ and $p \bar{p}$ collisions with the help of the ReBB model [36], limited to the investigated $0.541 \leq \sqrt{s} \leq 8 \mathrm{TeV}$ center of mass energy and in the $0.377 \leq-t \leq 1.2 \mathrm{GeV}^{2}$ four-momentum transfer range.

We extrapolate, in the $\mathrm{TeV}$ energy range, the $p p$ differential cross sections to energies where measured $p \bar{p}$ data exist and the other way round, the $p \bar{p}$ differential cross sec- 


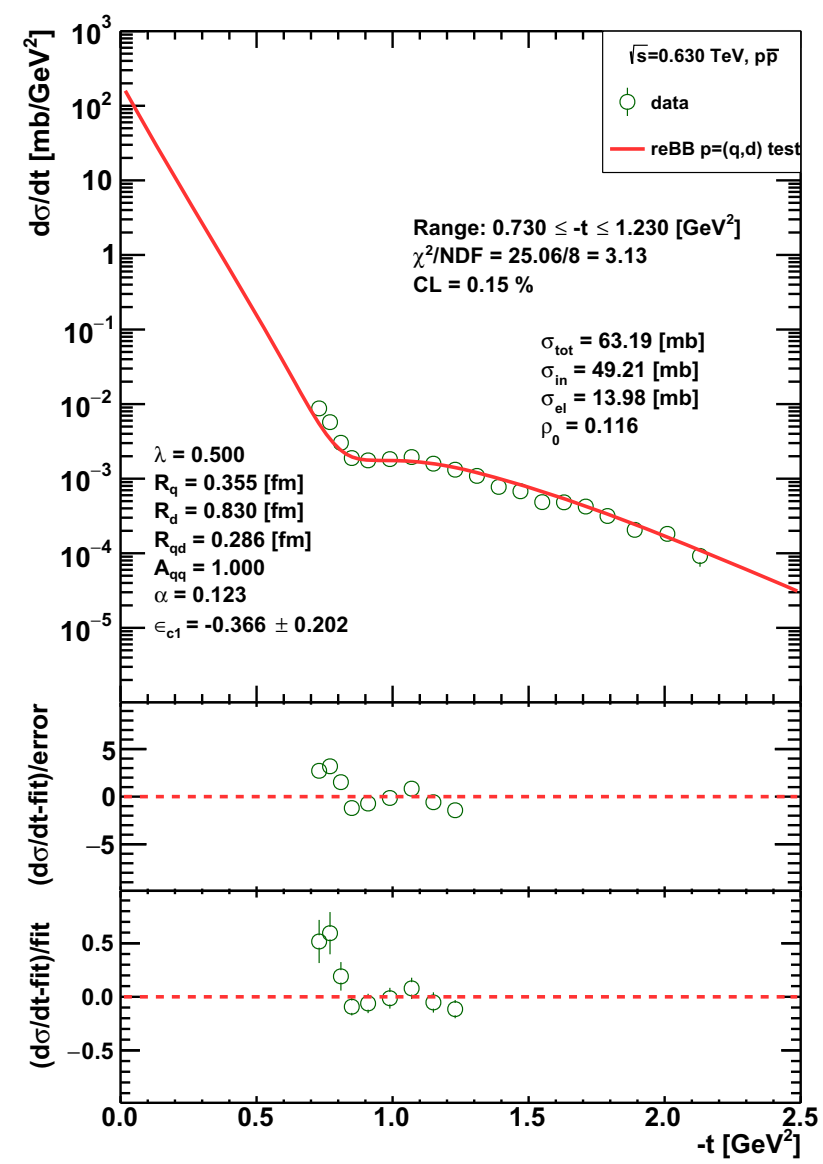

Fig. 9 Result of a sanity test, similar to Fig. 8, but for the $\sqrt{s}=0.63$ $\mathrm{TeV} p \bar{p}$ elastic differential cross section data of Ref. [66], fitted in the range $0.7 \leq-t \leq 1.2 \mathrm{GeV}^{2}$

tions to energies where measured $p p$ data exist. Thus three of such extrapolations were performed: $p p$ extrapolation to $\sqrt{s}=1.96 \mathrm{TeV}$, to compare it to the $1.96 \mathrm{TeV} \mathrm{D} 0 p \bar{p} d \sigma / d t$ data, and $p \bar{p}$ extrapolations to $\sqrt{s}=2.76$ and $7 \mathrm{TeV}$, to compare them to the $d \sigma / d t p p$ data measured by TOTEM at these energies.

Since the energy dependences of the scale parameters $R_{q}$, $R_{d}$ and $R_{q d}$ are identical for $p p$ and $p \bar{p}$ scattering, as discussed in Sect. 5, in the course of the extrapolations their values are fixed at their fitted values given in Table 1, furthermore, since the energy dependence of the $\alpha$ parameter differs for $p p$ and $p \bar{p}$ scattering, the $\alpha(p p)$ and $\alpha(p \bar{p})$ values are fixed from their energy dependence trend seen in Fig. 6d. In addition, during the extrapolations, the $\epsilon$ parameters in the $\chi^{2}$ definition, Eq. (6), were optimized, furthermore the last two terms in Eq. (6), i.e., the total cross section and $\rho_{0}$-parameter term, were not included. This way we handled the type $\mathrm{B}$ and type $\mathrm{C}$ errors of the published $p p$ differential cross-section to match these data as much as possible to the differential cross-section of elastic $p \bar{p}$ collisions within the allowed systematic errors, and vice versa.

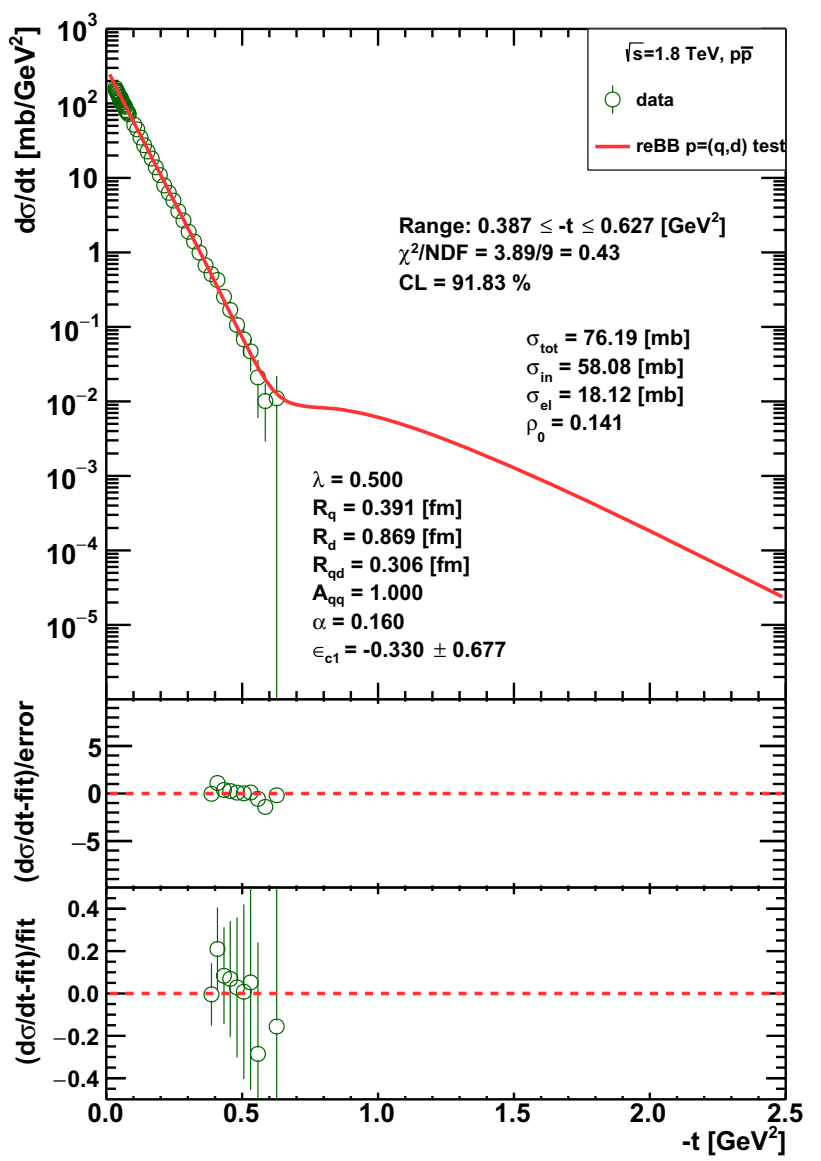

Fig. 10 Result of a sanity test, same as Fig. 8, but for the $1.8 \mathrm{TeV} p \bar{p}$ elastic differential cross section data [67] in the range of $0.37 \leq-t \leq$ $0.6 \mathrm{GeV}^{2}$

The results of the extrapolations are shown in Figs. 16, 17 and 18. The error band around these extrapolations is also evaluated, based on the envelope of one standard deviation errors of the $R_{q}(s), R_{d}(s), R_{q d}(s)$ model parameters and the $p_{0}$ and $p_{1}$ parameters of $\alpha(s)$. As an example, the resulting ten curves - considering that the values of the scale parameters are taken from the original fit while the value $\alpha$ is taken from the trend - are explicitly shown for $1.96 \mathrm{TeV}$ in Fig. 16.

While at $\sqrt{s}=1.96 \mathrm{TeV}$ no statistically significant difference is observed between the extrapolated $p p$ and measured $p \bar{p}$ differential cross sections, at $\sqrt{s}=2.76$ and 7 $\mathrm{TeV}$, remarkable and statistically significant differences can be observed. In Figs. 17 and 18, even an untrained eye can see, that the dip is filled in case of elastic $p \bar{p}$ scattering, while it is not filled in elastic $p p$ scattering. Thus we confirm the prediction of Ref. [69], that predicted, based on a three-gluon exchange picture that dominates at larger values of $-t$, that the dip will be filled in high energy $p \bar{p}$ elastic collisions.

In this work, the differences between elastic $p p$ and $p \bar{p}$ collisions are quantified by the confidence levels obtained from the comparision of the extrapolated curves to the mea- 


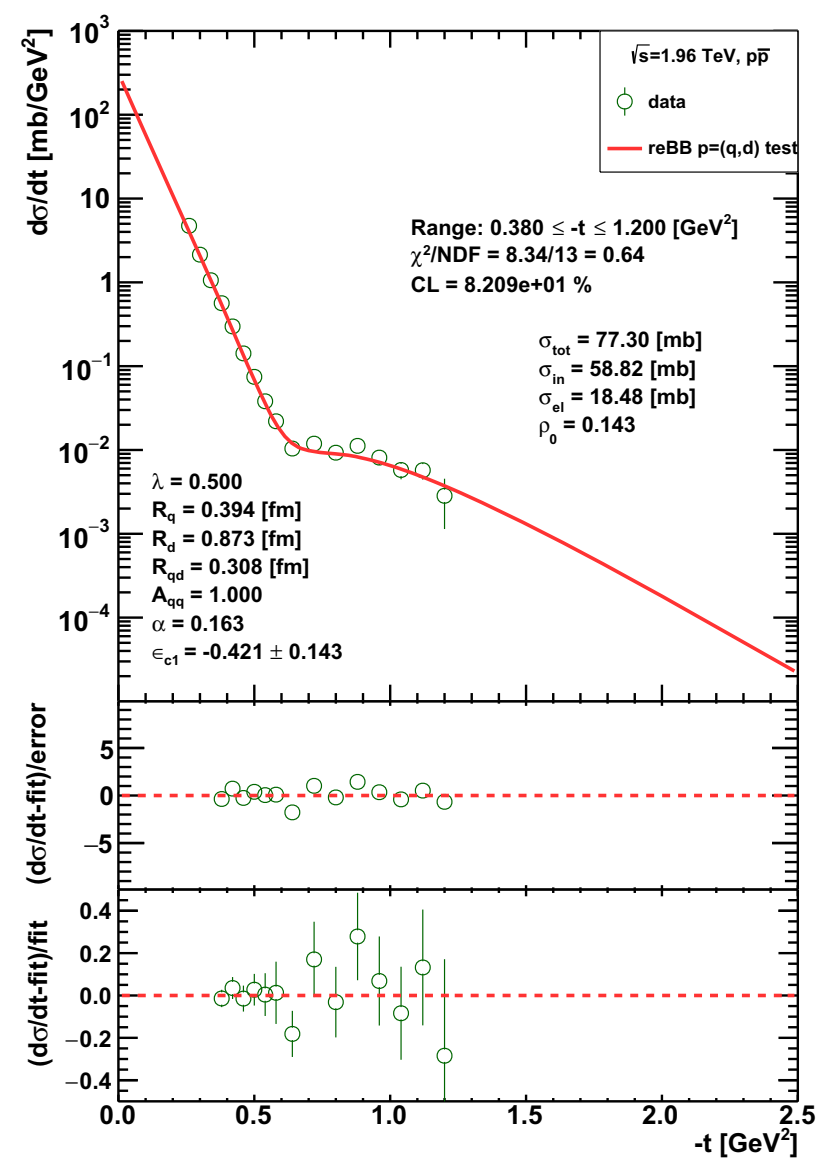

Fig. 11 Result of a sanity test, same as Fig. 8, but for the $\sqrt{s}=1.96$ $\mathrm{TeV} p \bar{p}$ elastic differential cross section data [45] in the range of $0.37 \leq$ $-t \leq 1.2 \mathrm{GeV}^{2}$

sured data: at $2.76 \mathrm{TeV}$, the hypothesis that these extrapolations agree with the data is characterized by a $C L=$ $1.092 \times 10^{-10} \%$, while at $7 \mathrm{TeV}, \mathrm{CL}=0 \%$. Theoretically the observed difference can be attributed only to the effect of a C-odd exchange, as detailed recently in Refs. [30-32]. At the $\mathrm{TeV}$ energy scale, the secondary Reggeon exchanges are generally known to be negligible. This effect has been also specifically cross-checked and confirmed recently in Ref. [70]. Thus in the few TeV energy range of the LHC, the only source of a difference between the differential cross-sections of elastic $p p$ and $p \bar{p}$ collisions can be a $t$-channel Odderon exchange. In the modern language of $\mathrm{QCD}$, the Odderon exchange corresponds to the exchange of C-odd colorless bound states consisting of odd number of gluons $[2,69,71]$.

Thus the CL, calculated for the $2.76 \mathrm{TeV} p \bar{p}$ extrapolation, corresponds to an Odderon observation with a probability of $P=1-C L=1-1.092 \times 10^{-12}$. This corresponds to a $\chi^{2} / N D F=100.35 / 20$ and to a $7.12 \sigma$ model dependent significance for the observation of a $t$-channel Odderon exchange, and the existence of the colorless bound states containing odd number of gluons. When extrapolat-

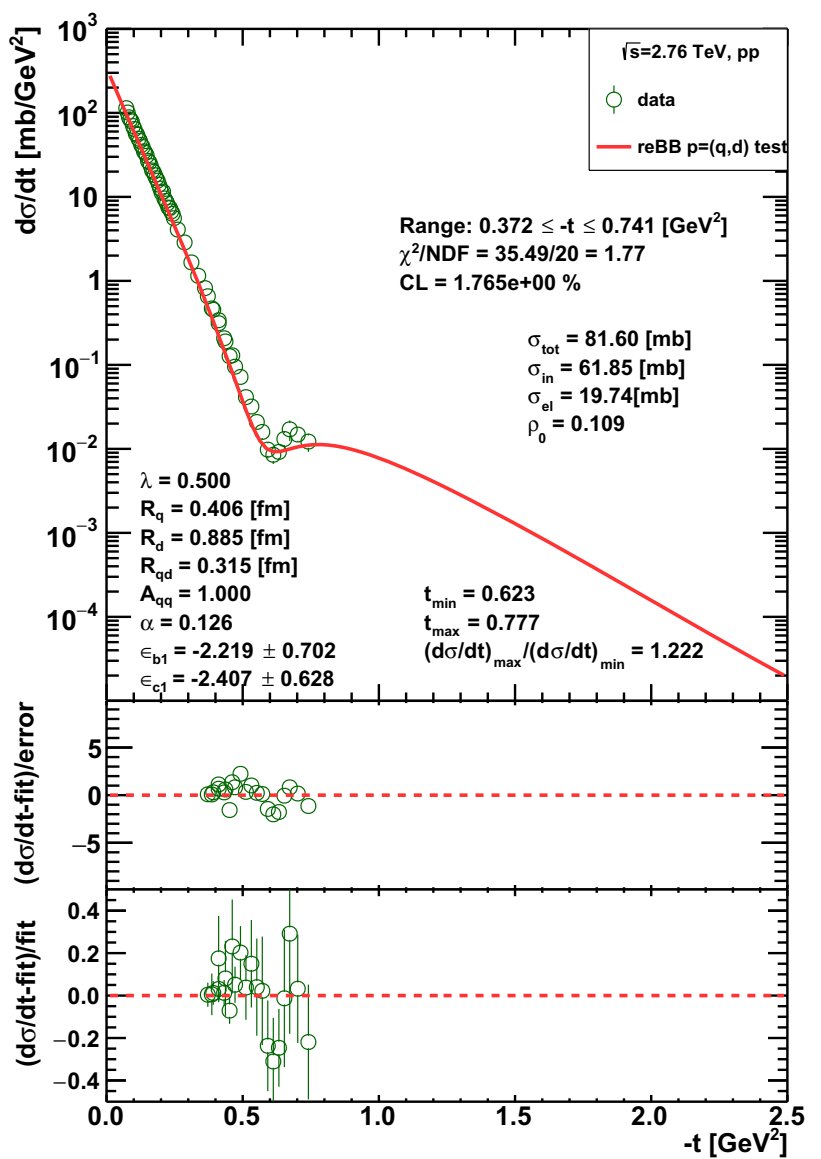

Fig. 12 Result of a sanity test, same as Fig. 8, but for the $\sqrt{s}=2.76$ $\mathrm{TeV} p p$ elastic differential cross section data [9] in the range of $0.37 \leq$ $-t \leq 0.7 \mathrm{GeV}^{2}$

ing the $p p$ differential cross-sections from 2.76 down to $1.96 \mathrm{TeV}$, however, significance is lost, corresponding to a $\chi^{2} / N D F=24.28 / 13$ and to a $2.19 \sigma$ effect, less than a $3 \sigma$ effect in this comparison. However, these two significances at 1.96 and $2.76 \mathrm{TeV}$ can be combined, providing a combined $\chi^{2} / N D F=124.63 / 33$, that corresponds to a statistically significant, $7.08 \sigma$ effect.

This $7.08 \sigma$ combined significance increases to an even larger significance of an Odderon observation, when we extrapolate the differential cross-section of elastic proton antiproton collisions to $\sqrt{s}=7.0 \mathrm{TeV}$, where the probability of Odderon observation becomes practically unity. Given that a $7.08 \sigma$ effect is already well above the usual $5 \sigma$, statistically significant discovery level, we quote this as the possibly lowest level of the significance of our model-dependent Odderon observation.

As already mentioned in the introduction we have also been recently involved in a truly model-independent search for Odderon effects in the comparision of the scaling properties of the differential cross-sections of elastic $p p$ and $p \bar{p}$ collisions in a similar $s$ but in the complete available $t$ range. 


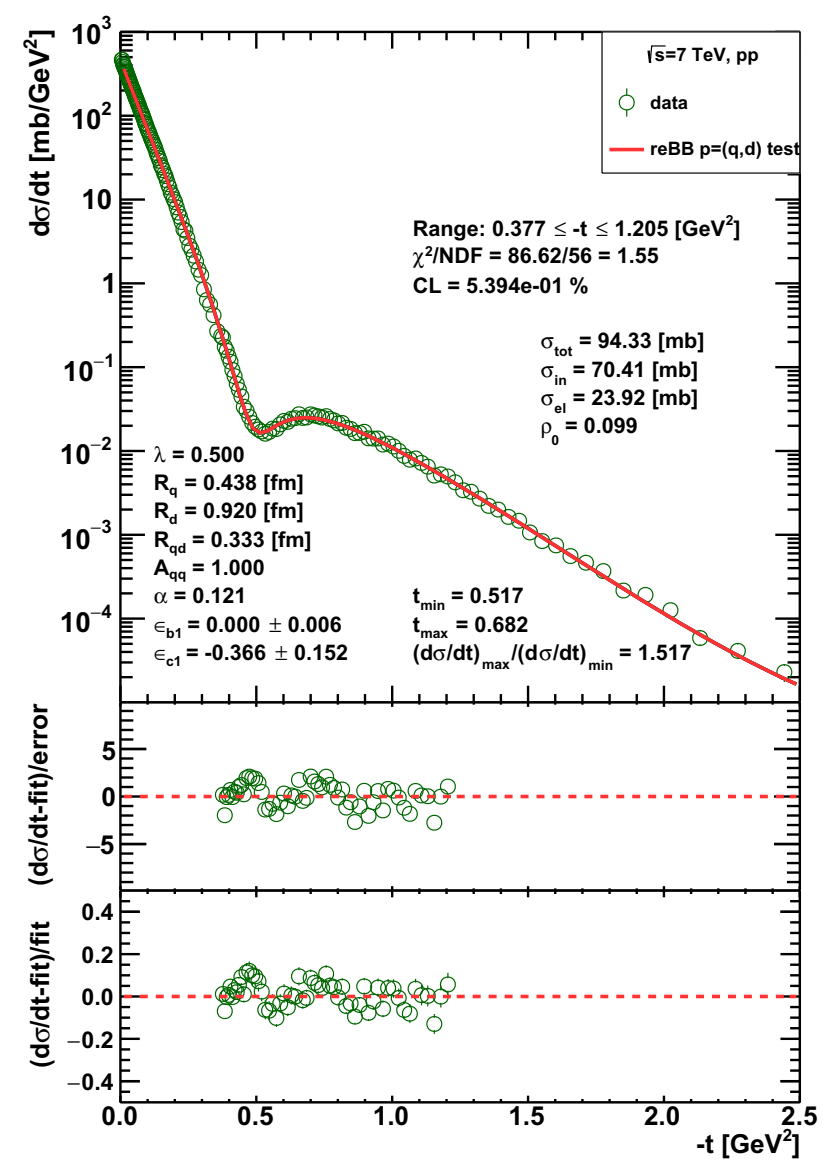

Fig. 13 Result of a sanity test, same as Fig. 8, but for the $p p$ elastic differential cross section data at $\sqrt{s}=7 \mathrm{TeV}$ from Ref. [47], in the fitted range of $0.37 \leq-t \leq 1.2 \mathrm{GeV}^{2}$

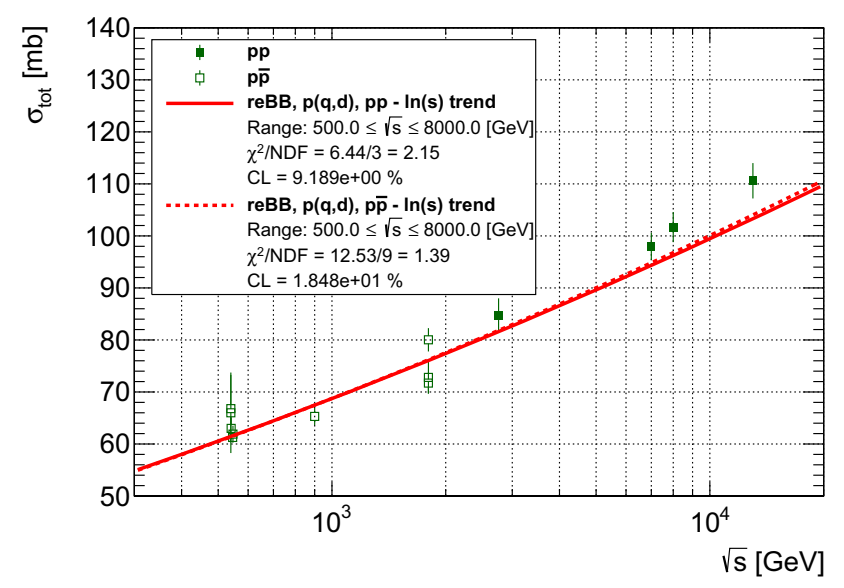

Fig. 14 Result of the sanity test for $p p[6,65,68]$ and $p \bar{p}[50]$ total cross section data. It was calculated from the model when the values of the parameters $R_{q}, R_{d}, R_{q d}$ and $\alpha$ were taken from Eq. 10 and Table 2, corresponding to the linear curves shown on panels (a)-(d) of Fig.6

As compared to the model-dependent studies summarized in this manuscript, the advantage of the model-independent scaling studies of Refs. [30-32] is that they scale out all the

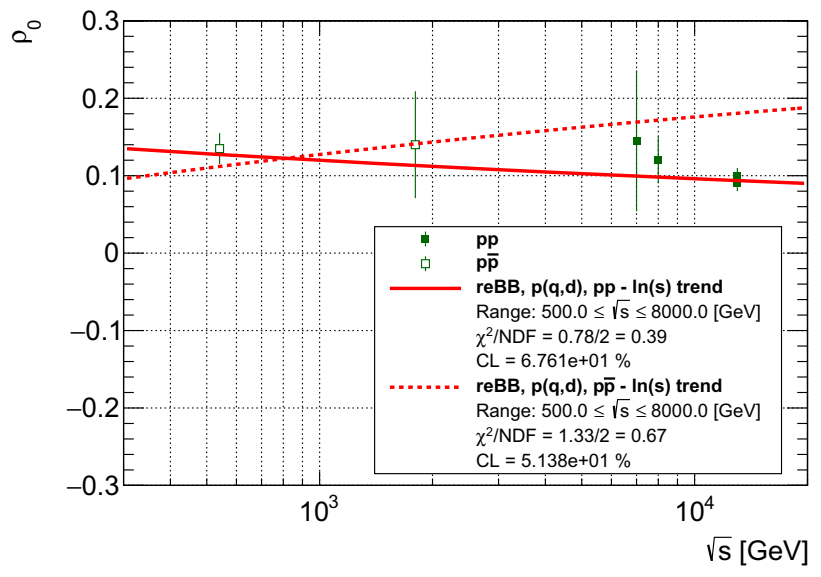

Fig. 15 Sanity test result for $p p[7,56,65]$ and $p \bar{p}[50]$ parameter $\rho_{0}$ data, as calculated from the model when the values of the parameters $R_{q}, R_{d}, R_{q d}$ and $\alpha$ were taken from Eq. (10) and Table 2, corresponding to the linear curves shown on panels (a)-(d) of Fig.6. On this plot, a model dependent Odderon effect is clearly identified: it corresponds to $\rho_{0}^{p p}(s) \neq \rho_{0}^{p \bar{p}}(s)$, the non-vanishing difference between the excitation functions of $\rho_{0}$ for $p p$ and $\rho_{0}$ for $p \bar{p}$ collisions, as detailed in Appendix $\mathrm{C}$

effects from the differences between $p p$ and $p \bar{p}$ elastic collisions due to possible differences in their $\sigma_{\mathrm{el}}(s), B(s)$ and their product, the $\sigma_{\mathrm{el}}(s) B(s)=\sigma_{\text {tot }}^{2}(s)\left(1+\rho_{0}^{2}(s)\right)$ functions. As part of the Odderon signal in the ReBB model is apparently in the difference between the $\rho_{0}(s)$ excitation functions for $p p$ and $p \bar{p}$ collisions, the significance of the Odderon signal is reduced in this model independent analysis. When considering the interpolations as theoretical curves, the significance is reduced to a $6.55 \sigma$ effect [30], but when considering that the interpolations between experimental data have also horizontal and vertical, type A and type B errors, the significance of the Odderon signal is further reduced to a $6.26 \sigma$ effect [31,32]. Thus we conclude that the Odderon is now discovered, both in a model-dependent and in a model-independent manner, with a statistical significance that is well above the $5 \sigma$ discovery limit of high energy particle physics.

Finally we close this section with the predictions to the experimentally not yet available large- $t$ differential crosssection of $p p$ collisions at $\sqrt{s}=0.9,4,5$ and $8 \mathrm{TeV}$ shown in Fig. 19.

\section{Discussion}

In the previous sections, we have investigated what happens if we interpret the data in terms of a particular model, the Real Extended Bialas-Bzdak Model. This allows also to consider the Odderon signal in the excitation function of the model parameter $\alpha$. We have shown in Appendix B that this model parameter is proportional to the experimentally measured parameter $\rho_{0}$, the ratio of the real to the imaginary part of 


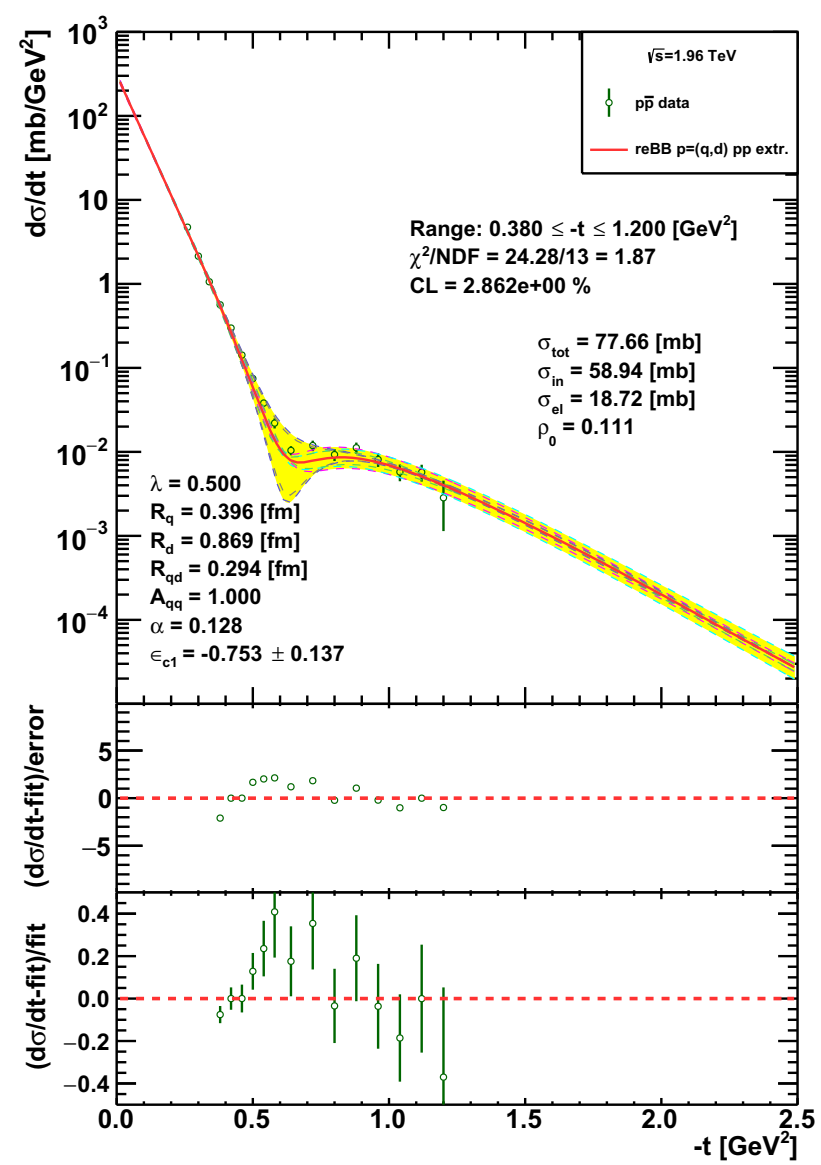

Fig. 16 The ReBB model extrapolation for the $p p d \sigma / d t$ at $\sqrt{s}=$ $1.96 \mathrm{TeV}$ compared to the $p \bar{p} \mathrm{D} 0 d \sigma / d t$ data [45] measured at the same energy. The yellow band is the uncertainty of the extrapolation. The calculated CL value between the extrapolated model and the measured data does not indicate a significant difference between the $p p$ and $p \bar{p}$ differential cross sections

the scattering amplitude at the optical point, and related the coefficient of proportionality to the value of the imaginary part of the scattering amplitude at vanishing impact parameter, $\lambda(s)=\operatorname{Imt}_{e l}(s, b=0)$, for the $\sqrt{s} \leq 8 \mathrm{TeV}$ elastic proton-proton collisions, and we have shown that within the framework of this ReBB model, the very different trend of $\rho_{0}(s)$ in proton-proton and in proton-antiproton collisions enhances the model-independent Odderon signal, from a 6.26 $\sigma$ and $6.55 \sigma$ effect to a combined, at least $7.08 \sigma$ effect.

Recently, the TOTEM Collaboration concluded, that only one condition is yet to be satisfied to see a statistically significant Odderon signal: namely, the logarithmically small energy gap between the lowest TOTEM energy of $\sqrt{s}=2.76$ $\mathrm{TeV}$ at LHC and the highest D0 energy of $1.96 \mathrm{TeV}$ at Tevatron needs to be closed. This energy gap has been closed in a model-independent way in Refs. [30-32], using the scaling properties of elastic scattering, and by comparing the $H(x)=\frac{1}{B \sigma_{e l}} \frac{d \sigma}{d t}$ scaling functions of elastic proton-proton

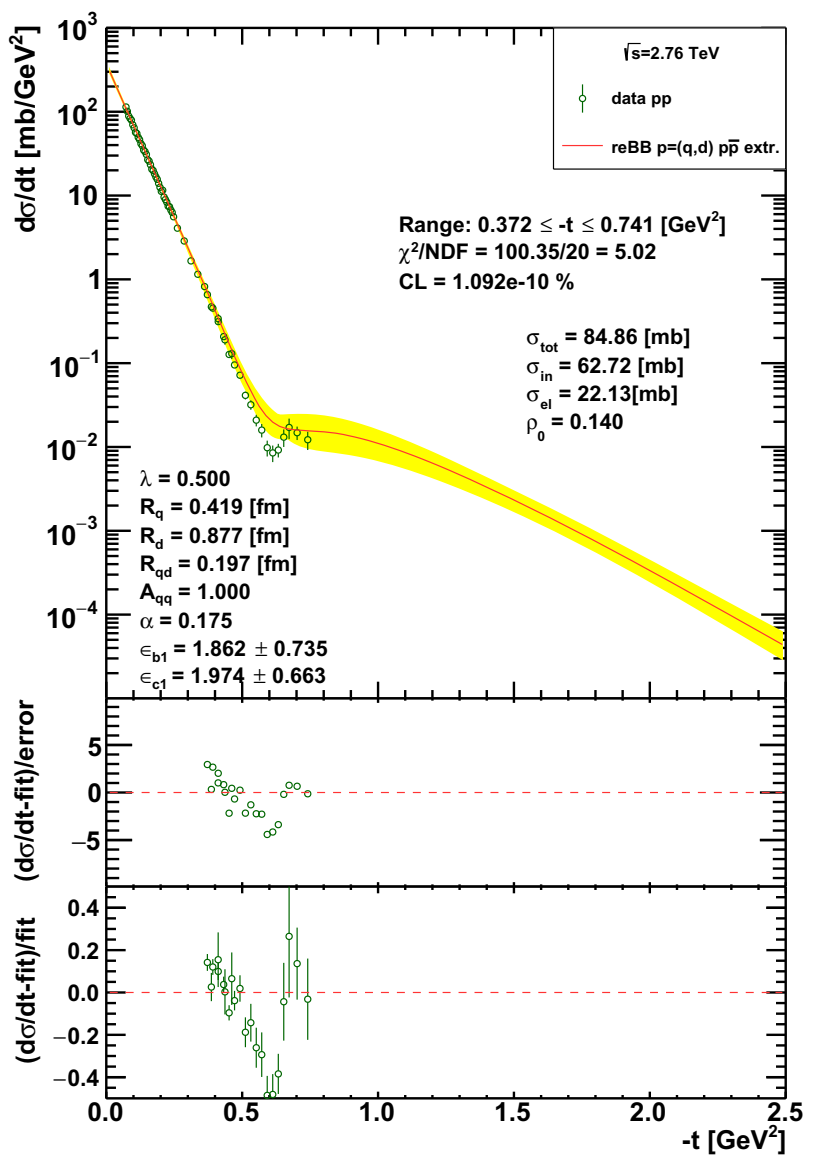

Fig. 17 The ReBB model extrapolation for the $p \bar{p} d \sigma / d t$ at $\sqrt{s}=$ $2.76 \mathrm{TeV}$ compared to the $p p$ TOTEM $d \sigma / d t$ data [9] measured at the same energy. The yellow band is the uncertainty of the extrapolation. The calculated CL value between the extrapolated model and the measured data indicates a significant difference between the $p p$ and $p \bar{p}$ differential cross sections, corresponding to a $7.1 \sigma$ significance for the $t$-channel Odderon exchange

and proton-antiproton collisions, as a function of $x=-t B$ at $\sqrt{s}=1.96,2.76$ and $7.0 \mathrm{TeV}$. The advantages of that method, with respect to comparing the cross sections directly include the scaling out of the $s$-dependencies of $\sigma_{\mathrm{el}}(s), B(s)$ and their product, $\sigma_{\mathrm{el}}(s) B(s)=\sigma_{\mathrm{tot}}^{2}(s)\left(1+\rho_{0}^{2}(s)\right)$, as well as the normalization of the $H(x)$ scaling function that cancels the point-to-point correlated and $t$-independent normalization errors. The validity of the $H(x)$ scaling for $p p$ collisions and its violation in $p \bar{p}$ collisions in the few $\mathrm{TeV}$ energy range resulted in a discovery level statistical significance of an Odderon signal, characterized in Refs. [30-32] to be at least 6.26 $\sigma$, model independently, based on a careful interpolation of the experimental data-points, their point-to-point fluctuating, point-to-point correlated and data point dependent as well as point-to-point correlated and data point independent errors. If these errors are considered as errors on a theory curve, then the significance goes up to at least $6.55 \sigma$ [30]. 


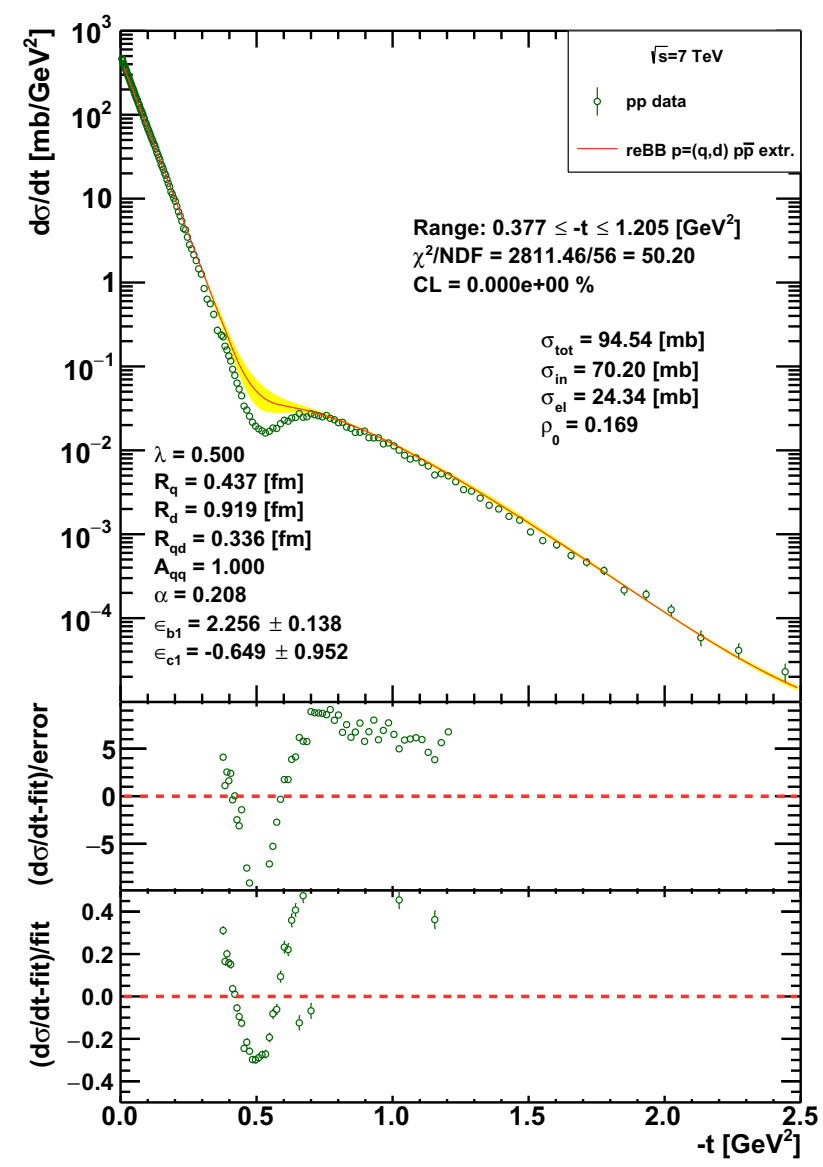

Fig. 18 The ReBB model extrapolation for the $p \bar{p} d \sigma / d t$ at $\sqrt{s}=$ $7 \mathrm{TeV}$ compared to the $p p$ TOTEM $d \sigma / d t$ data [47] measured at the same energy. The yellow band is the uncertainty of the extrapolation. The calculated CL value between the extrapolated model and the measured data indicates a significant difference between the $p p$ and $p \bar{p}$ differential cross sections, hence a significant Odderon effect, that is dominant around the dip region

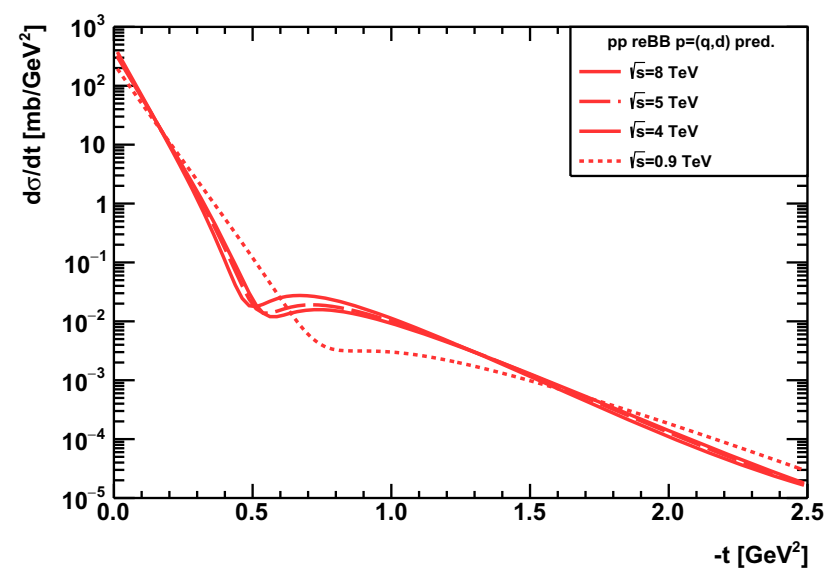

Fig. 19 Predictions from the ReBB model, for the $d \sigma / d t$ of elastic $p p$ collisions at $\sqrt{s}=8,5,4$, and $0.9 \mathrm{TeV}$
In high energy particle physics, the standard accepted discovery threshold corresponds to a $5 \sigma$ effect. In the previous section, we have shown, that the statistical significance of an Odderon observation in the limited $0.541 \leq \sqrt{s} \leq 8 \mathrm{TeV}$ center of mass energy and in the $0.377 \leq-t \leq 1.2 \mathrm{GeV}^{2}$ four-momentum transfer range is at least a combined $7.08 \sigma$ effect, corresponding to a statistically significant and model dependent Odderon observation.

The $\sqrt{s}=7 \mathrm{TeV} p p$ differential cross-sections are measured with asymmetric type B errors. In order to make sure that our results are reliable and reproducible, we have performed several cross-checks to test the reliability of our fit at $\sqrt{s}=7 \mathrm{TeV}$. One of these tests related to the handling of the asymmetric type $\mathrm{B}, t$-dependent systematic errors. We have performed cross-checks for taking at every point either the smaller or the larger of the up and down type B errors to have a lower or an upper limit on their effects. We found that the parameters of the ReBB model remained stable for such a symmetrization of the type B systematic errors, as the modification of the fit parameters due to such a symmetrization was within the quoted errors on the fit parameters. Our final fits, presented before, were done with asymmetric type $\mathrm{B}$ errors, as detailed in Sect. 4. So we conclude that our fit at $\sqrt{s}=7 \mathrm{TeV}$ is stable even for the symmetrization of the type B systematic errors.

We have also investigated the stability of our result for the case, when the energy range is extended towards lower values of $\sqrt{s}$, in the ISR energy range, detailed in Appendix D. When the $\sqrt{s}=23.5 \mathrm{GeV}$ energy data are included to those summarized in Table 1, the energy dependence of the model parameters becomes quadratic in $\ln (s)$. This provides $3 \times 5=15$ model parameters for this broader energy range, as summarized in Table 3 and detailed in Appendix D. This way, the non-linear terms are confirmed to be negligibly small in the $\mathrm{TeV}$ energy range, where we find the significant Odderon effects, with the help of as little as only 10 model parameters. These 10 parameters are given in Table 2.

It turns out in Sect. 4, that the ReBB model as presented in Ref. [36] does not yet provide a statistically acceptable fit quality to the differential cross-section of $\sqrt{s}=13 \mathrm{TeV}$ elastic $p p$ scattering. This might be due to the emergence of the black-ring limit of elastic proton-proton scattering instead of the expected black-disc limit. In what follows we shortly discuss the earlier and more recent results on the black ring shaped interaction region of the colliding protons.

A complementary way of studying the high-energy scattering processes is by passing from the momentum transfer $t$ to the impact parameter $b$. In 1963 van Hove introduced the inelasticity profile or the overlap function $[72,73]$, which corresponds to the impact parameter distribution of the inelastic cross section characterizing the shape of the interaction region of two colliding particles. The natural expectation is that the most inelastic collisions are central, i.e., the inelas- 
Table 3 Summary of the parameter values which determine the energy dependence according to the quadratic dependence in $\ln (s)$ by Eq. (D.1). The values of the parameters are rounded up to three valuable decimal digits except for $p_{2}$ that are rounded up to four valuable decimal digits.

\begin{tabular}{llllll}
\hline Parameter & $R_{q}(f m)$ & $R_{d}(f m)$ & $R_{q d}(f m)$ & $\alpha(p p)$ & $\alpha(p \bar{p})$ \\
\hline$\chi^{2} / N D F$ & $2.829 / 2$ & $0.273 / 2$ & $1.870 / 2$ & $0.760 / 2$ & $1.212 / 2$ \\
CL $(\%)$ & 24.31 & 87.23 & 39.25 & 0.68 & 54.54 \\
$p_{0}$ & $0.235 \pm 0.010$ & $0.651 \pm 0.017$ & $0.383 \pm 0.037$ & $-0.209 \pm 0.031$ & $0.060 \pm 0.041$ \\
$p_{1}$ & $0.004 \pm 0.002$ & $0.010 \pm 0.003$ & $-0.021 \pm 0.008$ & $0.048 \pm 0.006$ & $-0.005 \pm 0.007$ \\
$p_{2}$ & $0.0005 \pm 0.0001$ & $0.0003 \pm 0.0001$ & $0.0010 \pm 0.0003$ & $-0.0017 \pm 0.0003$ & $0.0008 \pm 0.0003$ \\
\hline
\end{tabular}

ticity profiles have a maximum at $b=0$ consistently with the black disc terminology. The possibility of a minimum at $b=0$, i.e., the peripheral form of the inelastic function was first considered in Ref. [74] which implies the shape of a black ring rather than that of a black disc.

In Ref. [58], it was shown that the inelasticity profile of protons is governed by the ratio of the slope of the diffraction cone to the total cross section through the variable $Z=4 \pi B / \sigma_{\text {tot }}$ and the evolution to values of $Z<1$ at LHC energies implies a transition from the black disk picture of the interaction region to a black ring (or torus-like) shape. These results were reviewed in Reference [59] using the unitarity relation in combination with experimental data on elastic scattering in the diffraction cone. Reference [58] concludes that the shape of the interaction region of colliding protons could be reliably determined if the behavior of the elastic scattering amplitude at all transferred momenta was known.

The black ring shape of the interaction region can be interpreted as the presence of a hollow at small impact parameter values.

In Refs. [75-78] the authors study the hollowness phenomenon within an inverse scattering approach based on empirical parameterizations. Reference [76] concludes that the very existence of the hollowness phenomenon is quantummechanical in nature. Hollowness has also been reported to emerge from a gluonic hot-spot picture of the $p p$ collision at the LHC energies [60]. It is shown in Ref. [78] that the emergence of such a hollow strongly depends on the phase of the scattering amplitude. In Ref. [79] the authors demonstrated the occurrence of the hollowness phenomenon in a Regge model above $\sqrt{s} \sim 3 \mathrm{TeV}$.

Reference [61] discusses the absorptive (saturation of the black disk limit) and reflective (saturation of the unitarity limit) scattering modes of proton-proton collisions concluding that a distinctive feature of the transition to the reflective scattering mode is the developing peripheral form of the inelastic overlap function. Reflective scattering is detailed also in Refs. [80-82].
These parameters are also shown on the panels of Fig. 30. For $R_{q}, R_{d}$ and $R_{q d}$, the values of the parameters $p_{0}, p_{1}$ and $p_{2}$ are given in units of femtometers (fm). For the parameters $\alpha(p p)$ and $\alpha(p \bar{p})$, the parameters $p_{0}, p_{1}$ and $p_{2}$ are dimensionless

The authors of Ref. [29] argue that the presence of nonzero real part of the elastic scattering amplitude in the unitarity condition enables to conserve the traditional black disk picture refuting the existence of the hollowness effect. However, as noted in Ref. [79], the criticism that has been raised in Ref. [29] is based on an incorrect perception of the approximations involved and does not address the arbitrariness of the $t$-dependence of the ratio $\rho$ which is crucial for hollowness.

In Refs. $[83,84]$ the hollowness effect is interpreted as a consequence of fundamental thermodynamic processes.

Reference [57] notes that the onset of the hollowness effect is possibly connected to the opening of a new channel between $\sqrt{s}=2.76$ and $7 \mathrm{TeV}$ indicating by the measured $\sigma_{e l} / \sigma_{t o t}$ ratio and the slope parameter $B_{0}$ data.

In Ref. [85] the model independent Lévy imaging method is employed to reconstruct the proton inelasticity profile function and its error band at different energies. This method established a statistically significant proton hollowness effect, well beyond the $5 \sigma$ discovery limit. This conclusion is based on a model independent description of the TOTEM proton-proton differential cross-section data at $\sqrt{s}=13 \mathrm{TeV}$ with the help of the Lévy imaging method, that represents the TOTEM data in a statistically acceptable manner, corresponding to a confidence level of $\mathrm{CL}=2 \%$.

\section{Summary}

Currently, the statistically significant observation of the elusive Odderon is a hot research topic, with several interesting and important results and contributions. In the context of this manuscript, Odderon exchange corresponds to a crossingodd component of the scattering amplitude of elastic protonproton and proton-antiproton collisions, that does not vanish at asymptotically high energies, as probed experimentally by the D0 Collaboration for proton-antiproton and by the TOTEM Collaboration for proton-proton elastic collisions in the $\mathrm{TeV}$ energy range. Theoretically, the observed differences can be attributed only to the effect of a C-odd exchange, as detailed recently in Refs. [30-32]. Those model 
independent studies resulted in an at least $6.26 \sigma$ statistical significance of the Odderon exchange [30-32]. The goal of the research summarized in this manuscript was to crosscheck, in a model-dependent way, the persistence of these Odderon-effects, and to provide a physical picture to interpret these results. Using the ReBB model of Ref. [36], developed originally to describe precisely the differential crosssection of elastic proton-proton collisions, we were able to describe also the proton-antiproton differential cross section at $\sqrt{s}=0.546$ and $1.96 \mathrm{TeV}$ without any modification of formalism. We have shown also that this model describes the proton-proton differential cross section at $\sqrt{s}=2.76$ and 7 $\mathrm{TeV}$, also in a statistically acceptable manner, with a CL > $0.1 \%$.

Using our good quality, statistically acceptable fits for the $0.5 \leq \sqrt{s} \leq 8 \mathrm{TeV}$ energy region, we have determined the energy dependence of the model parameters to be an affine linear function of $\ln \left(s / s_{0}\right)$. We have verified this energy dependence by demonstrating that the excitation functions of the physical parameters of the Real Extended Bialas-Bzdak model satisfy the so-called sanity tests: they describe in a statistically acceptable manner not only those four datasets that formed the basis of the determination of the excitation function, but all other published datasets in the $\sqrt{s}=0.541-$ 8.0 $\mathrm{TeV}$ energy domain. We have also demonstrated that the excitation functions for the total cross-sections and the $\rho_{0}$ ratios correspond to the experimentally established trends.

Remarkably, we have observed that the energy dependence of the geometrical scale parameters for $p p$ and $p \bar{p}$ scattering are identical in elastic proton-proton and protonantiproton collisions: only the energy dependence of the shape or opacity parameter $\alpha(s)$ differs significantly between $p p$ and $p \bar{p}$ collisions. After determining the energy dependence of the model parameters we made extrapolations in order to compare the $p p$ and $p \bar{p}$ differential cross sections in the few $\mathrm{TeV}$ energy range, corresponding to the energy of D0 measurement at $\sqrt{s}=1.96 \mathrm{TeV}$ in Ref. [45] and the TOTEM measurements at $\sqrt{s}=2.76$ and 7.0 TeV. Doing this, we found evidence for the Odderon exchange with a high statistical significance. We have cross-checked, that this evidence withstands several reasonable cross-checks, for example the possible presence of small quadratic terms of $\ln \left(s / s_{0}\right)$ in the excitation functions of the parameters of this model. Subsequently, we have also predicted the details of the diffractive interference (dip and bump) region at $\sqrt{s}=0.9,4,5$ and 8 $\mathrm{TeV}^{1}$

We have shown that within the framework of this ReBB model, the very different trend of $\rho_{0}(s)$ in proton-proton and in proton-antiproton collisions enhances the model-

\footnotetext{
${ }^{1}$ Currently, TOTEM preliminary experimental data are publicly presented from an on-going analysis at $\sqrt{s}=8 \mathrm{TeV}$, see Ref. [54] for further details.
}

independent Odderon signal, from a $6.26 \sigma$ and $6.55 \sigma$ effect of Refs. [30-32] to an at least $7.08 \sigma$ effect. This gain of significance is due to the possibility of extrapolating the differential cross-sections of elastic $p \bar{p}$ scattering from $\sqrt{s}=$ $1.96 \mathrm{TeV}$ to $2.76 \mathrm{TeV}$. It is important to note that in the evaluation of the $7.08 \sigma$ Odderon effect, only $p \bar{p}$ data at $\sqrt{s}=1.96$ $\mathrm{TeV}$ and $p p$ data at $\sqrt{s}=2.76 \mathrm{TeV}$ were utilized, amounting to a model dependent but successful closing of the energy gap between D0 and TOTEM measurements. Let us also emphasize that our Odderon observation is valid in the limited kinematic range of $0.541 \leq \sqrt{s} \leq 8 \mathrm{TeV}$ center of mass energy and in the $0.377 \leq-t \leq 1.2 \mathrm{GeV}^{2}$ four-momentum transfer range.

When extrapolating the $p p$ differential cross-sections from 2.76 down to $1.96 \mathrm{TeV}$, however, significance is lost, corresponding to a $\chi^{2} / N D F=24.28 / 13$ and to a $2.19 \sigma$ effect, which is less than a $3 \sigma$ effect at $1.96 \mathrm{TeV}$. However, these two significances at 1.96 and $2.76 \mathrm{TeV}$ can be combined, providing a $\chi^{2} / N D F=124.63 / 33$, that corresponds to a statistically significant, combined $7.08 \sigma$ effect.

This $7.08 \sigma$ combined significance increases to an even larger significance of an Odderon observation, when we extrapolate the differential cross-section of elastic protonantiproton collisions to $\sqrt{s}=7.0 \mathrm{TeV}$. Given that a $7.08 \sigma$ effect is already well above the usual $5 \sigma$, statistically significant discovery level, we quote this as the possibly lowest level of the significance of our model-dependent Odderon observation.

Concerning the direction of future research: Odderon is now discovered both in a model-independent way, described in Refs. [30-32], and in a model-dependent way, described in this manuscript; so the obvious next step is to extract its detailed properties, both in a model-independent and in a model-dependent manner. The main properties of the Odderon as well as the Pomeron, based on the ReBB model, are already summarized in Appendix $C$.

Let us also note, that the ReBB model as presented in Ref. [36] does not yet provide a statistically acceptable fit quality to the differential cross-section of $\sqrt{s}=13 \mathrm{TeV}$ elastic $p p$ scattering. This might be due to the emergence of the blackring limit of elastic proton-proton scattering instead of the expected black-disc limit, as detailed in Sect. 8, or due to the very strong non-exponential features of the differential cross-sections in these collisions at low $-t^{2}$, as shown in Refs. [6,7].

So we conclude that the Real Extended Bialas-Bzdak model needs to be further generalized for the top LHC energies and above. This work is in progress, but it goes

\footnotetext{
2 We see that the ReBB model has a leading order exponential feature. If we want to describe the significantly non-exponential features of differential cross-section in the low- $|t|$ range $[7,55]$, the model has to be generalized for a possible non-exponential behaviour at low $|t|$.
} 
clearly well beyond the scope of the current, already rather detailed manuscript. Importantly, any possible outcome of these follow-up studies is not expected to modify the model behavior at the presently investigated energy range, and hence our work is apparently completed, refinements are not necessary from the point of view of the task solved in this manuscript.

In short, we determined the model-dependent statistical significance of the Odderon observation to be an at least $7.08 \sigma$ effect in the $0.5 \leq \sqrt{s} \leq 8 \mathrm{TeV}$ center of mass energy and $0.377 \leq-t \leq 1.2 \mathrm{GeV}^{2}$ four-momentum transfer range. Our analysis is based on the analysis of published D0 and TOTEM data of Refs. $[6,9,45]$ and uses as a tool the Real Extended Bialas-Bzdak model of Ref. [36]. We have cross-checked that this unitary model works in a statistically acceptable, carefully tested and verified manner in this particular kinematic range. Our main results are illustrated on Figs. 17 and 18.

Acknowledgements First of all, we would like to thank F. Nemes, who initiated this project, and started to test the Real Extended BialasBzdak model against the proton-antiproton data. He also provided several valuable technical help for us in the initial phases of these studies. We greatfully acknowledge inspiring discussions with C. Avila, S. Giani, P. Grannis, G. Gustafson, L. Jenkovszky, E. Levin, B. Nicolescu, T. Novák, K. Österberg, R. Pasechnik, C. Royon, A. Ster and M. Strikman. Our research has been partially supported by the ÚNKP-18-2 New National Excellence Program of the Ministry of Human Capacities, and by the NKFIH Grants no. FK-123842, FK-123959 and K133046 as well as by the EFOP 3.6.1-16-2016-00001 Grant (Hungary), as well as by the framework of the COST Action CA15213 "Theory of hot matter and relativistic heavy-ion collisions (THOR)" of the European Union.

Data Availability Statement This manuscript has no associated data or the data will not be deposited. [Authors' comment: This manuscript contains a meta-analysis of already published data, and in this sense no new measurements are reported here. Our observation of odderon exchange is based on a new analysis of already published data, that are already part of the public domain.]

Open Access This article is licensed under a Creative Commons Attribution 4.0 International License, which permits use, sharing, adaptation, distribution and reproduction in any medium or format, as long as you give appropriate credit to the original author(s) and the source, provide a link to the Creative Commons licence, and indicate if changes were made. The images or other third party material in this article are included in the article's Creative Commons licence, unless indicated otherwise in a credit line to the material. If material is not included in the article's Creative Commons licence and your intended use is not permitted by statutory regulation or exceeds the permitted use, you will need to obtain permission directly from the copyright holder. To view a copy of this licence, visit http://creativecomm ons.org/licenses/by/4.0/.

Funded by SCOAP ${ }^{3}$.

\section{Appendix A: Basics of the Bialas-Bzdak model and its unitary extension}

In the followings, let us shortly introduce the details of the $p=(q, d)$ ReBB model based on Refs. [36,39].

The elastic scattering amplitude in the $b$ impact parameter space can be written in the so called eikonal form as:

$t_{e l}(s, b)=i\left[1-e^{-\Omega(s, b)}\right]$,

where $\Omega(s, b)$ is the opacity or eikonal function. In general this opacity is a complex valued function $[42,86]$. The shadow profile function is given as

$P(s, b)=1-|\exp (-\Omega)|^{2}=\tilde{\sigma}_{i n}(s, b)$,

and this is the reason why the shadow profile function is also frequently called as the inelastic profile function, as it describes the probability distribution of inelastic collisions in the impact parameter space. This way the inelastic $p p$ scattering may be characterized by a probability distribution. However, let us stress that elastic scattering is an inherently quantum process, as evidenced by a diffractive interference that results in diffractive minima and maxima of the differential cross-sections. Probabilistic interpretation can be given only to the inelastic scattering, or to the sum of elastic scattering plus propagating without interactions.

If the real part of the scattering amplitude can be neglected, then the $\Omega(s, b)$ has only a real part given as

$\operatorname{Re} \Omega(s, b)=-\frac{1}{2} \ln \left[1-\tilde{\sigma}_{i n}(s, b)\right]$.

The inelastic profile function was evaluated with the help of Glauber's multiple diffraction theory [42] for the colliding protons consisting a constituent quark and diquark or $p=$ $(q, d)$ picture in Section 2.2 of Ref. [36] and the results were visualized in Figs. 5 and 9 of that paper.

The imaginary part of the opacity function in Ref. [36], which generates the real part of the scattering amplitude, is defined to be proportional to the inelastic scattering probability,

$\operatorname{Im} \Omega(s, b)=-\alpha \cdot \tilde{\sigma}_{i n}(s, b)$,

were $\alpha$, mentioned earlier, is a free parameter and proportional to $\rho_{0}$ (see Appendix B). This ansatz assumes that the inelastic collisions at low four-momentum transfers correspond to the cases when the parts of proton suffer elastic scattering but these parts are scattered to different directions, not parallel to one another. Other models were also tested on TOTEM data in Ref. [36], but this physically motivated assumption worked well and was shown to be consistent with the experimental data at $\sqrt{s}=7 \mathrm{TeV}$ in Ref. [36]. 


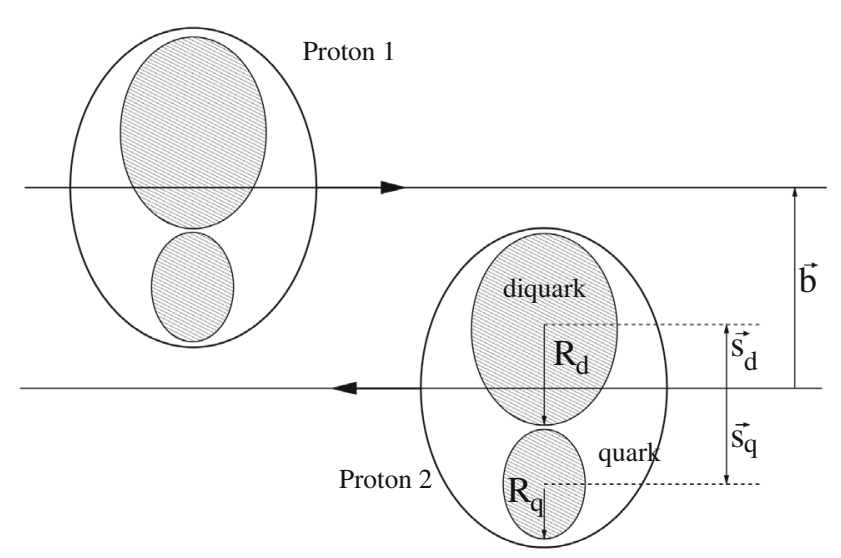

Fig. 20 Visualization of the proton-proton scattering in the quarkdiquark BB model. The figure is taken from Ref. [33]

The inelastic scattering probability in the BB model [39] for a fixed impact parameter $\mathbf{b}$ as a probability distribution, given as

$$
\begin{aligned}
\tilde{\sigma}_{i n}(b)= & \int_{-\infty}^{+\infty} \cdots \int_{-\infty}^{+\infty} d^{2} \mathbf{s}_{q} d^{2} \mathbf{s}_{q}^{\prime} d^{2} \mathbf{s}_{d} d^{2} \mathbf{s}_{d}^{\prime} D\left(\mathbf{s}_{q}, \mathbf{s}_{d}\right) D\left(\mathbf{s}_{q}^{\prime}, \mathbf{s}_{d}^{\prime}\right) \\
& \times \sigma\left(\mathbf{s}_{q}, \mathbf{s}_{d} ; \mathbf{s}_{q}^{\prime}, \mathbf{s}_{d}^{\prime} ; b\right)
\end{aligned}
$$

where $\mathbf{s}_{q}, \mathbf{s}_{q}^{\prime}, \mathbf{s}_{d}$ and $\mathbf{s}_{d}^{\prime}$ are the transverse positions of the quarks and diquarks in the two colliding protons (see Fig. 20). $D\left(\mathbf{s}_{q}, \mathbf{s}_{d}\right)$ denotes the distribution of quark and diquark inside the proton which is considered to be Gaussian:

$D\left(\mathbf{s}_{q}, \mathbf{s}_{d}\right)=\frac{1+\lambda^{2}}{R_{q d}^{2} \pi} e^{-\left(s_{q}^{2}+s_{d}^{2}\right) / R_{q d}^{2}} \delta^{2}\left(\mathbf{s}_{d}+\lambda \mathbf{s}_{q}\right)$,

where $\lambda=m_{q} / m_{d}$ is the ratio of the quark and diquark masses, furthermore $R_{q d}$ is the standard deviation of the quark and diquark distance emerging as a free parameter. The two-dimensional Dirac $\delta$ function fixes the center-ofmass of the proton and reduces the dimension of the integral in Eq. (A.5) from eight to four. The diquark positions can be expressed by that of the quarks:

$\mathbf{s}_{d}=-\lambda \mathbf{s}_{q}, \mathbf{s}_{d}^{\prime}=-\lambda \mathbf{s}_{q}^{\prime}$.

The term $\sigma\left(\mathbf{s}_{q}, \mathbf{s}_{d} ; \mathbf{s}_{q}^{\prime}, \mathbf{s}_{d}^{\prime} ; b\right)$ is the probability of inelastic interactions at a fixed impact parameter and transverse positions of all constituents and given by a Glauber expansion as follows:

$$
\sigma\left(\mathbf{s}_{q}, \mathbf{s}_{d} ; \mathbf{s}_{q}^{\prime}, \mathbf{s}_{d}^{\prime} ; b\right)=1-\prod_{a} \prod_{b}\left[1-\sigma_{a b}\left(\mathbf{b}+\mathbf{s}_{a}^{\prime}-\mathbf{s}_{b}\right)\right]
$$

where $a, b \in\{q, d\}$. The terms $\sigma_{a b}$ (s) are the inelastic differential cross-sections of the binary collisions of the con- stituents having Gaussian shapes:

$\sigma_{a b}(\mathbf{s})=A_{a b} e^{-s^{2} / S_{a b}^{2}}, S_{a b}^{2}=R_{a}^{2}+R_{b}^{2}, \quad a, b \in\{q, d\}$.

where $R_{q}, R_{d}$ and $A_{a b}$ are free parameters. The physical meaning of the $R_{q}$ and $R_{d}$ parameters as well as the impact parameter $b$ and the coordinates $s_{q}$ and $s_{d}$ is illustrated on Fig. 20 and detailed in Ref. [36].

The inelastic quark-quark, quark-diquark and diquarkdiquark cross sections are obtained by integrating Eq. (A.9):

$\sigma_{a b, \text { inel }}=\int_{-\infty}^{+\infty} \int_{-\infty}^{+\infty} \sigma_{a b}(\mathbf{s}) \mathrm{d}^{2} s=\pi A_{a b} S_{a b}^{2}$.

The number of the free parameters of the model can be reduced demanding that the ratios of the cross sections are

$\sigma_{q q, \text { inel }}: \sigma_{q d, \text { inel }}: \sigma_{d d, \text { inel }}=1: 2: 4$,

expressing the idea that the constituent diquark contains twice as many partons than the constituent quark and also that the colliding constituents do not "shadow" each other.

Using Eq. (A.10) and the assumptions given by Eq. (A.11), the $A_{q d}$ and $A_{d d}$ parameters can be expressed through $A_{q q}$ :

$A_{q d}=A_{q q} \frac{4 R_{q}^{2}}{R_{q}^{2}+R_{d}^{2}}, A_{d d}=A_{q q} \frac{4 R_{q}^{2}}{R_{d}^{2}}$.

Counting the number of free parameters one finds that the model now contains six of them: $R_{q}, R_{d}, R_{q d}, \alpha, \lambda$ and $A_{q q}$. However, it was shown in Ref. [36] that the latter two parameters can be fixed. $\lambda=0.5$ if the diquark is very weakly bound, so that its mass is twice as large as that of the valence quark. The Real Extended Bialas Bzdak model describes the experimental data in the $\sqrt{s} \leq 8 \mathrm{TeV}$ region with $A_{q q}=1$ fixed, assuming that head-on $q q$ collisions are inelastic with a probability of 1, corresponding to Eq. (A.9).

Substituting Eq. (A.3) and Eq. (A.4) to Eq. (A.1) one obtains for the scattering amplitude:

$t_{e l}(s, b)=i\left(1-e^{i \alpha \tilde{\sigma}_{i n}(s, b)} \sqrt{1-\tilde{\sigma}_{i n}(s, b)}\right)$.

This equation is, in fact, a special solution of the unitarity relation, obtained from the optical theorem. The integral for $\tilde{\sigma}_{i n}(s, b)$ defined by Eq. (A.5) can be calculated analytically with the methods described in Refs. [36,39].

In order to compare the theoretical model to the experimental data, the amplitude in impact parameter space, given by Eq. (A.13), has to be transformed into momentum space by a Fourier-Bessel transformation:

$T(s, t)=2 \pi \int_{0}^{\infty} J_{0}(\Delta \cdot b) t_{e l}(s, b) b \mathrm{~d} b$.

In the above formula $b=|\mathbf{b}|, \Delta=|\boldsymbol{\Delta}|$ is the transverse momentum and $J_{0}$ is the zeroth order Bessel-function of 
the first kind. Here the high energy limit is considered, i.e., $\sqrt{s} \rightarrow \infty$ and then $\Delta(t) \simeq \sqrt{-t}$.

Substituting the expression for the elastic scattering amplitude given by Eq. (A.14) into Eqs. (1)-(5) the model for fitting the scattering data is complete. The Fourier-Bessel integral in the amplitude can be calculated numerically during the fitting procedure.

\section{Appendix B: On the proportionality between $\rho_{0}(s)$ and $\alpha(s)$ in the ReBB model}

Let us first of all note that the detailed description of the Real Extended Bialas-Bzdak (ReBB) model is given in Section 2.2 of Ref. [36] and also summarized in Appendix A. We have utilized this formalism throughout the fits described in the body of the manuscript, however in this Appendix, we need to develop this formalism a bit further, as in the earlier publications the details of the relations between the $\rho_{0}$ parameter (the ratio of the real to the imaginary part of the scattering amplitude at $t=0$ ) and the parameter $\alpha$ of the ReBB model (that is responsible for filling up the singular dip of the original Bialas-Bzdak model of Refs. [37-40]) has not yet been detailed before.

Let us stress, that ReBB model is unitary, by definition. Thus the elastic scattering amplitude in the ReBB model too has unitary form given by Eq. (A.1), where the opacity function $\Omega(s, b)$ is, in general, a complex valued function.

In the ReBB model, the impact parameter dependent scattering amplitude is given by Eq. (A.13). Now we develop two small set of approximations that are based on the physical domain of the ReBB model parameters. From the fits performed so far, we always find $\alpha \lesssim 0.165$, corresponding to Table 1 of Ref. [36] and Table 1 of the current manuscript.

In the physical case, when $\alpha \tilde{\sigma}_{i n}(s, b) \ll 1$ is small, one obtains for the real and imaginary parts of the scattering amplitude, respectively,

$\operatorname{Re} t_{e l}(s, b) \simeq \alpha \tilde{\sigma}_{i n}(s, b) \sqrt{1-\tilde{\sigma}_{i n}(s, b)}$

and

$\operatorname{Im} t_{e l}(s, b) \simeq 1-\sqrt{1-\tilde{\sigma}_{i n}(s, b)}$.

Given that the real part of the scattering amplitude is thus proportional to $\alpha$ while the imaginary part is independent of $\alpha$, we indeed find that

$\rho_{0} \propto \alpha, \quad$ if $\alpha \ll 1$.

Based on Figs. 5 and 9. of Ref. [36] and the modelindependent results of the Levy series method detailed in Ref. [85], if the colliding energy is in the $\sqrt{s} \leq 8 \mathrm{TeV}$ domain, corresponding to the domain of our extrapolations, the shadow profile function is nearly Gaussian. Such a behaviour can be obtained easily as follows.
Let us approximate the imaginary part of the scattering amplitude with a Gaussian, i.e.,

$\operatorname{Im} t_{e l}(s, b) \simeq \lambda(s) \exp \left(-\frac{b^{2}}{2 R^{2}(s)}\right)$,

where $\lambda(s) \simeq \operatorname{Im} t_{e l}(s, b=0)$. Then the inelastic profile or shadow profile function takes the form of

$\tilde{\sigma}_{i n}(s, b)=2 \lambda(s) \exp \left(-\frac{b^{2}}{2 R^{2}(s)}\right)-\lambda(s)^{2} \exp \left(-\frac{b^{2}}{R^{2}(s)}\right)$

This expression, up to second order terms, starts as a Gaussian, but it actually corresponds to the subtraction of a broader and smaller Gaussian from a narrower and larger Gaussian in the physical domain of $\lambda(s) \leq 1$.

As $P_{0} \equiv P(s, 0)=\tilde{\sigma}_{\text {inel }}(s, b=0)$ is the value of the profile or inelastic profile function at $b=0$, we find the following relation between $P_{0}$ and $\lambda(s)$ :

$P_{0}(s)=2 \lambda(s)-\lambda^{2}(s) \leq 1$.

When performing the transformation from the impact parameter space to momentum space, the result for the real to imaginary part ratio of the forward scattering amplitude, defined by Eq. (5), is

$\rho_{0}(s)=\alpha(s)\left(2-\frac{3}{2} \lambda(s)+\frac{1}{3} \lambda^{2}(s)\right)$.

In the above equation, we may consider that $\lambda \equiv \lambda(s)$ is a function of $P_{0}(s)$ based on Eq. (B.6). Based on the formalism of Section 2.2 of Ref. [36], $P_{0} \equiv P_{0}(s)$ is a function of $R_{q}(s), R_{d}(s)$ and $R_{q d}(s)$ only, but otherwise it is independent of the fourth physical parameter of the Real Extended Bialas-Bzdak model, $\alpha(s)$. Hence the excitation function of $P_{0}(s)$ is determined completely by the parameters $p_{1}$ and $p_{0}$ of the excitation functions of the scale parameters ( $\left.R_{q}, R_{d}, R_{q d}\right)$, as summarized in Table 2 . This way, the $P_{0}=P_{0}\left(R_{q}(s), R_{d}(s), R_{q d}(s)\right)$ function is uniquely given by with the help of Eq. (A.13), corresponding to Eq. (29) of Ref. [36].

We have cross-checked the result of these analytic considerations compared to the fit results on $\alpha(s)$ and the measured values of $\rho_{0}(s)$ at the ISR energies and we find an excellent agreement between the analytic approximations and the numerical results at ISR, corresponding to the $\lambda(s)$ range of $0.73-0.78$, as illustrated in Fig. 32. The linear relationship between $\rho_{0}$ and the ReBB model parameter $\alpha$ is also indicated at the ISR energy range, in Fig. 31. Similarly, we find an excellent agreement between the analytic calculations of Eq. (B.7) and the numerical and experimental results at the energy scale of $0.5 \leq \sqrt{s} \leq 8 \mathrm{TeV}$, as demonstrated on Fig. 7, presented in the body of this manuscript. 


\section{Appendix C: Pomeron and Odderon from the Real Extended Bialas-Bzdak model}

In this Appendix we summarize, for the sake of clarity, how we can determine the crossing-even and crossing-odd components of the scattering amplitude, based on the ReBB model. In the TeV energy range, we indentify these components with the Pomeron and the Odderon amplitude, given that the Reggeon contributions in this energy range are generally expected to be negligibly small, as confirmed also by explicit calculations for example in Ref. [79].

In this energy range, the proton-proton $(p p)$ as well as the proton-antiproton $(p \bar{p})$ elastic scattering amplitudes can be written as

$$
\begin{aligned}
& T_{e l}^{p p}=T_{e l}^{+}-T_{e l}^{-}, \\
& T_{e l}^{p \bar{p}}=T_{e l}^{+}+T_{e l}^{-},
\end{aligned}
$$

where we have suppressed the dependence of these amplitudes on the Mandelstam variables: $T_{e l}^{p p} \equiv T_{e l}^{p p}(s, t)$ etc.

If the $p p$ and the $p \bar{p}$ scattering amplitudes are known, then the crossing even and the crossing odd components of the elastic scattering amplitude can be reconstructed as

$$
\begin{aligned}
T_{e l}^{+} & =\frac{1}{2}\left(T_{e l}^{p \bar{p}}+T_{e l}^{p p}\right), \\
T_{e l}^{-} & =\frac{1}{2}\left(T_{e l}^{p \bar{p}}-T_{e l}^{p p}\right) .
\end{aligned}
$$

In this manuscript, we have utilized the Real Extended Bialas-Bzdak or ReBB model of Ref. [36], to determine the elastic scattering amplitude for elastic $p p$ and $p \bar{p}$ scattering. This model is based on Glauber's theory of multiple diffractive scattering [41-43], and assumes that the elastic protonproton scattering is based on multiple diffractive scattering of the constituents of the protons. Hence this ReBB model has two main variants: the case when the proton is assumed to have a constituent quark and a diquark component is referred to as $p=(q, d)$ model, while the case when the diquark is assumed to be further resolved as a weakly bound state of two constituent quarks is the $p=(q,(q, q))$ model. It was shown before that this $p=(q,(q, q))$ variant predicts too many diffractive minima for the differential cross-section, hence in this paper we utilize the $p=(q, d)$ variant as formulated in Ref. [36], without any change.

With the help of the ReBB model of Ref. [36], we have described in a statistically acceptable manner the $p p$ and $p \bar{p}$ differential cross-sections. In this ReBB model the $p p$ elastic scattering amplitude depends on $s$ only through four energy dependent parameters, that we denote here, for the sake of clarity, as $R_{q}^{p p}(s), R_{d}^{p p}(s), R_{q d}^{p p}(s)$ and $\alpha^{p p}(s)$ :

$$
T_{e l}^{p p}(s, t)=F\left(R_{q}^{p p}(s), R_{d}^{p p}(s), R_{q d}^{p p}(s), \alpha^{p p}(s) ; t\right) .
$$

Similarly, we described the amplitude of the elastic $p \bar{p}$ scattering with 4 energy dependent parameters, that we denote here for the sake of clarity as $R_{q}^{p \bar{p}}(s), R_{d}^{p \bar{p}}(s), R_{q d}^{p \bar{p}}(s)$ and $\alpha^{p \bar{p}}(s)$ :

$T_{e l}^{p \bar{p}}(s, t)=F\left(R_{q}^{p \bar{p}}(s), R_{d}^{p \bar{p}}(s), R_{q d}^{p \bar{p}}(s), \alpha^{p \bar{p}}(s) ; t\right)$.

Here $F$ stands for a symbolic short-hand notation for a function, that indicates how the left hand side of the $p p$ and $p \bar{p}$ scattering amplitude depend on $s$ through their $s$ dependent parameters. The scale parameters $R_{q}, R_{d}$, and $R_{q d}$ correspond to the Gaussian sizes of the constituent quarks, diquarks and their separation in the scattering (anti)protons. Each of these parameters is $s$-dependent. Since the trends of $R_{q}(s), R_{d}(s)$ and $R_{q d}(s)$ follow, within errors, the same excitation functions in both $p p$ and $p \bar{p}$ collisions, as indicated on panels a, b and c of Fig. 6, we have denoted these in principle different scale parameters with the same symbols in the body of the manuscript:

$$
\begin{aligned}
R_{q}(s) & \equiv R_{q}^{p p}(s)=R_{q}^{p \bar{p}}(s), \\
R_{d}(s) & \equiv R_{d}^{p p}(s)=R_{d}^{p \bar{p}}(s), \\
R_{q d}(s) & \equiv R_{q d}^{p p}(s)=R_{q d}^{p \bar{p}}(s) .
\end{aligned}
$$

On the other hand, the opacity or dip parameters $\alpha(s)$ are different in elastic $p p$ and $p \bar{p}$ reactions: if they too were the same, then the scattering amplitude for $p p$ and $p \bar{p}$ reactions were the same, correspondingly the differential crosssections were the same in these reactions, while experimental results indicate that they are qualitatively different. Hence

$\alpha^{p p}(s) \neq \alpha^{p \bar{p}}(s)$,

corresponding to panel d of Fig. 6 and to Table 2.

In this form, the ReBB model of Ref. [36] provides a statistically acceptable description of the elastic scattering amplitude, both for $p p$ and $p \bar{p}$ elastic scattering, in the kinematic range that extends to at least $0.372 \leq-t \leq 1.2 \mathrm{GeV}^{2}$ and $0.546 \leq \sqrt{s} \leq 8 \mathrm{TeV}$. Now, for the sake of clarity, let us note that the $s$-dependence of the Pomeron and Odderon (crossing-even and crossing-odd) components of the scattering amplitude thus happens through the $s$-dependence of five parameters only: Based on Eqs. (C.3, C.4), Eqs. (C.5, C.6) and Eqs. (C.7, C.8,C.9) we find that

$$
\begin{aligned}
T_{e l}^{\mathbb{P}}(s, t) & =T_{e l}^{+}(s, t) \\
& =G\left(R_{q}^{p p}(s), R_{d}^{p p}(s), R_{q d}^{p p}(s), \alpha^{p p}(s), \alpha^{p \bar{p}}(s) ; t\right), \\
T_{e l}^{\mathbb{Q}}(s, t) & =T_{e l}^{-}(s, t) \\
& =H\left(R_{q}^{p p}(s), R_{d}^{p p}(s), R_{q d}^{p p}(s), \alpha^{p p}(s), \alpha^{p \bar{p}}(s) ; t\right) .
\end{aligned}
$$


Here $G$ and $H$ are just sympolic short-hand notations that summarize how the left hand side of the above equations depend on $s$ through their $s$-dependent parameters.

The differential cross section, Eq. (1), the total, elastic and inelastic cross sections, Eqs.(2)-(4), as well as the real to imaginary ratio, Eq. (5), and the nuclear slope parameter,

$B(s, t)=\frac{d}{d t} \ln \frac{d \sigma(s, t)}{d t}$,

characterize experimentally the $(s, t)$ dependent elastic scattering amplitudes, $T_{e l}(s, t)$ discussed above. These quantities can be evaluated for a specific process like the elastic $p p$ or $p \bar{p}$ scattering. Given that we evaluate the elastic scattering amplitude for both of them in the TeV energy range, that yields also the $(s, t)$ dependent elastic scattering amplitude also for the Pomeron and the Odderon exchange, we have the possibility to evaluate these quantities for the crossingeven Pomeron $(\mathbb{P})$ and for the crossing-odd Odderon $(\mathbb{O})$ exchange.

The momentum space dependent scattering amplitude $T_{e l}(s, t)$, for spin independent processes, is related to a Fourier-Bessel transform of the impact parameter dependent elastic scattering amplitudes $t_{e l}(s, b)$ as given by Eq. (A.14).

This impact parameter dependent amplitude is constrained by the unitarity of the scattering matrix $S$,

$S S^{\dagger}=I$

where $I$ is the identity matrix. Its decomposition is $S=$ $I+i T$, where the matrix $T$ is the transition matrix. In terms of $T$, unitarity leads to the relation

$T-T^{\dagger}=i T T^{\dagger}$

which can be rewritten in terms of the impact parameter or $b$ dependent amplitude $t_{e l}(s, b)$ as

$2 \mathcal{I}_{m} t_{e l}(s, b)=\left|t_{e l}(s, b)\right|^{2}+\tilde{\sigma}_{\text {inel }}(s, b)$,

where $\tilde{\sigma}_{\text {inel }}(s, b)$ is the impact parameter dependent probability of inelastic scattering. It can be equivalently expressed from the above unitarity relation as

$\tilde{\sigma}_{\text {inel }}(s, b)=1-\left(\mathcal{R e}_{e l}(s, b)\right)^{2}-\left(\mathcal{I}_{m} t_{e l}(s, b)-1\right)^{2}$.

It follows that

$\tilde{\sigma}_{\text {inel }}(s, b) \leq 1$

as a consequence of unitarity.

Given that the ReBB model of Ref. [36] is unitary, those dispersion relations that are consequences of the unitarity of the scattering amplitude are automatically satisfied. For example, the dispersion relations discussed in Refs. [87,88] are automatically satisfied by the unitary ReBB model.

The impact parameter dependent elastic scattering amplitudes for elastic $p p$ and $p \bar{p}$ scatterings are given in terms of the complex opacity or eikonal functions $\Omega(s, b)$. The defining relations are

$t_{e l}^{p p}(s, b)=i\left(1-\exp \left(-\Omega^{p p}(s, b)\right)\right.$,

$t_{e l}^{p \bar{p}}(s, b)=i\left(1-\exp \left(-\Omega^{p \bar{p}}(s, b)\right)\right.$.

As another consequence of the unitary relations, we have

$\sqrt{1-\tilde{\sigma}_{\text {inel }}(s, b)}=\exp (-\mathcal{R} e \Omega(s, b))$.

In Ref. [36], three different possibilities were considered for the solution of the unitarity relation, using various functions to model the imaginary part of the complex opacity function $\Omega$, that corresponds to the real part of the scattering amplitude. Out of the considered three possible choices, the assumption that was found to be consistent with the experimental data on $p p$ elastic scattering at the ISR and LHC energies is defined by Eq. (A.13). At that time it was not yet clear that a similar relation works also for $p \bar{p}$ collisions. A very important advantage of this particular solution to the unitarity equation is that the multiple diffractive scattering theory of R. J. Glauber predicts $\tilde{\sigma}_{\text {inel }}(s, b)$ to depend only on the $s$-dependent geometrical scales $\left(R_{q}(s), R_{d}(s), R_{q d}(s)\right)$. Given that the $R_{q}(s), R_{d}(s), R_{q d}(s)$ scales are found in panels a, b, and c of Fig. 6 to be independent of the type of the elastic collisions i.e. to be the same in elastic $p p$ and $p \bar{p}$ collisions in the body of this paper, the imaginary part of the complex opacity function in elastic $p p$ and $p \bar{p}$ collisions has the same $b$-dependent factor, but has an $s$-dependent prefactor that is in principle a different function in the cases of elastic $p p$ and $p \bar{p}$ collisions:

$\mathcal{I} m \Omega^{p p}(s, b)=-\alpha^{p p}(s) \tilde{\sigma}_{i n e l}(s, b)$,

$\mathcal{I} m \Omega^{p \bar{p}}(s, b)=-\alpha^{p \bar{p}}(s) \tilde{\sigma}_{\text {inel }}(s, b)$.

These relations yield the following simple expressions for the impact parameter dependent elastic $p p$ and $p \bar{p}$ scattering amplitudes

$t_{e l}^{p p}(s, b)=i\left(1-e^{i \alpha^{p p}(s) \tilde{\sigma}_{i n}(s, b)} \sqrt{1-\tilde{\sigma}_{i n}(s, b)}\right)$,

$t_{e l}^{p \bar{p}}(s, b)=i\left(1-e^{i \alpha^{p \bar{p}}(s) \tilde{\sigma}_{i n}(s, b)} \sqrt{1-\tilde{\sigma}_{i n}(s, b)}\right)$.

It then clearly follows that in the ReBB model $t_{e l}^{p p}(s, b) \equiv$ $t_{e l}^{p \bar{p}}(s, b)$ and $t_{e l}^{\mathbb{O}}(s, b) \equiv 0$ if, and only if $\alpha^{p p}(s) \equiv \alpha^{p \bar{p}}(s)$.

As detailed in Appendix B, $\alpha(s) \sim \rho_{0}(s)$ both for $p p$ and $p \bar{p}$ elastic collisions. At the same time $\alpha(s)$ controls the value of the differential cross section in the region of the dip in these collisions. Thus, within the ReBB model there is a deep connection between the $t=0$ and the dip region. This supports the findings that the recently observed decrease in $\rho_{0}(s)$ around $\sqrt{s}=13 \mathrm{TeV}$, the dip-bump structure in $p p$ 
scattering and its absence in $p \bar{p}$ scattering are both the consequences of the Odderon contribution. In the ReBB model, this Odderon contribution is encoded in the difference between $\alpha^{p p}(s)$ and $\alpha^{p \bar{p}}(s)$. This conclusion is supported also by the detailed calculations of the ratios of the modulus squared Odderon to Pomeron scattering amplitudes.

Thus if $\rho_{0}^{p p}(s) \neq \rho_{0}^{p \bar{p}}(s)$, within the ReBB model it follows that $\alpha^{p p}(s) \neq \alpha^{p \bar{p}}(s)$ or equivalently $t_{e l}^{\mathbb{Q}}(s, b) \neq 0$ in the $\mathrm{TeV}$ region.

Within the framework of the ReBB model, we thus can significantly sharpen an Odderon theorem noted in Ref. [30]. The weaker, original form of this theorem was formulated in Ref. [30] as follows:

\section{Theorem 1}

- If the $p p$ differential cross sections differ from that of $p \bar{p}$ scattering at the same value of $s$ in a TeV energy domain, then the Odderon contribution to the scattering amplitude cannot be equal to zero, i.e.

$$
\frac{d \sigma^{p p}}{d t} \neq \frac{d \sigma^{p \bar{p}}}{d t} \text { for } \sqrt{s} \geq 1 \mathrm{TeV} \Longrightarrow T_{\mathrm{el}}^{O}(s, t) \neq 0 .
$$

This theorem is model-independently true as it depends only on the general structure of the theory of elastic scattering. The outline of the proof is that the differential cross-section, Eq. (1), is proportional to the modulus squared elastic scattering amplitudes both for $p p$ and $p \bar{p}$ scattering. If the modulus square of two complex functions is different, then the two complex functions, corresponding to the elastic scattering amplitudes of $p p$ and $p \bar{p}$ collisions, cannot be identical. Hence their difference, proportional to the Odderon amplitude in the $\mathrm{TeV}$ energy range, cannot be zero.

Within the ReBB model, this theorem can be significantly sharpened. This sharpened, stronger version of the above theorem thus reads as follows:

Theorem 2: In the framework of the unitary Real Extended Bialas-Bzdak (ReBB) model, the elastic $p p$ differential cross sections differ from that of elastic $p \bar{p}$ scattering at the same value of $s$ in a TeV energy domain, if and only if the Odderon contribution to the scattering amplitude is not equal to zero. This happens if and only if $\alpha^{p p}(s) \neq \alpha^{p \bar{p}}(s)$ and as a consequence, if and only if $\rho_{0}^{p p} \neq \rho_{0}^{p \bar{p}}$ :

$$
\begin{aligned}
& \frac{d \sigma^{p p}}{d t} \neq \frac{d \sigma^{p \bar{p}}}{d t} \Longleftrightarrow T_{\mathrm{el}}^{O}(s, t) \neq 0 \\
& \Longleftrightarrow \rho_{0}^{p p}(s) \neq \rho_{0}^{p \bar{p}}(s) \\
& \Longleftrightarrow \alpha^{p p}(s) \neq \alpha^{p \bar{p}}(s) \\
& \text { for } \sqrt{s} \geq 1 \mathrm{TeV} .
\end{aligned}
$$

This theorem is proven by the explicit expressions for the impact parameter dependent elastic scattering amplitude for the C-even Pomeron and the C-odd Odderon exchange in the ReBB model as detailed below. These relations are consequences of the unitarity of the ReBB model.

$t_{e l}^{\mathbb{P}}(s, b)=i\left(1-\frac{1}{2}\left(\exp \left(-\Omega^{p p}(s, b)\right)+\exp \left(-\Omega^{p \bar{p}}(s, b)\right)\right)\right)$,

$t_{e l}^{\mathbb{Q}}(s, b)=i \frac{1}{2}\left(\exp \left(-\Omega^{p p}(s, b)\right)-\exp \left(-\Omega^{p \bar{p}}(s, b)\right)\right)$.

It is obvious to note that the Pomeron amplitude given above is crossing-even, while the Odderon amplitude is crossingodd: $C t_{e l}^{\mathbb{P}}(s, b)=t_{e l}^{\mathbb{P}}(s, b)$ and $C t_{e l}^{\mathbb{Q}}(s, b)=-t_{e l}^{\mathbb{Q}}(s, b)$.

These relations can be equivalently rewritten for the Pomeron amplitude, using the shorthand notation $\tilde{\sigma}_{i n} \equiv$ $\tilde{\sigma}_{i n}(s, b)$ and suppressing the $s$ dependencies of $\alpha^{p p}(s)$ and $\alpha^{p \bar{p}}(s)$, as follows:

$$
\begin{aligned}
& \operatorname{Im}_{t_{e l}^{\mathbb{P}}}(s, b)=1-\sqrt{1-\tilde{\sigma}_{i n}} \cos \left(\frac{\alpha^{p p}+\alpha^{p \bar{p}}}{2} \tilde{\sigma}_{i n}\right) \cos \left(\frac{\alpha^{p \bar{p}}-\alpha^{p p}}{2} \tilde{\sigma}_{i n}\right), \\
& \mathcal{R} e t_{e l}^{\mathbb{P}}(s, b)=\sqrt{1-\tilde{\sigma}_{i n}} \sin \left(\frac{\alpha^{p p}+\alpha^{p \bar{p}}}{2} \tilde{\sigma}_{i n}\right) \cos \left(\frac{\alpha^{p \bar{p}}-\alpha^{p p}}{2} \tilde{\sigma}_{i n}\right) .
\end{aligned}
$$

This form of the Pomeron amplitude is explicitly C-even, as corresponding to the Pomeron amplitude in unitary, Real Extended Bialas-Bzdak model. Thus if the difference between the opacity parameters $\alpha$ for $p p$ and $p \bar{p}$ elastic collisions is small, the Pomeron is predominantly imaginary, with a small real part that is proportional to $\sin \left(\frac{\alpha^{p p}+\alpha^{p \bar{p}}}{2} \tilde{\sigma}_{i n}\right)$. Similarly, for the Odderon, we have in the ReBB model the following amplitude

$$
\begin{gathered}
\operatorname{Ret}_{e l}^{\mathbb{Q}}(s, b)=\sqrt{1-\tilde{\sigma}_{i n}} \sin \left(\frac{\alpha^{p \bar{p}}-\alpha^{p p}}{2} \tilde{\sigma}_{i n}\right) \cos \left(\frac{\alpha^{p \bar{p}}+\alpha^{p p}}{2} \tilde{\sigma}_{i n}\right), \\
(\mathrm{C} .27) \\
\mathcal{I m}_{e l}^{\mathbb{Q}}(s, b)=\sqrt{1-\tilde{\sigma}_{i n}} \sin \left(\frac{\alpha^{p \bar{p}}-\alpha^{p p}}{2} \tilde{\sigma}_{i n}\right) \sin \left(\frac{\alpha^{p p}+\alpha^{p \bar{p}}}{2} \tilde{\sigma}_{i n}\right) .
\end{gathered}
$$

This form of the Odderon amplitude is explicitly C-odd and satisfies unitarity, corresponding to the Real Extended Bialas-Bzdak model. If the difference between the opacity parameters $\alpha$ for $p p$ and $p \bar{p}$ elastic collisions becomes vanishingly small, both the real and the imaginary part of the Odderon amplitude vanishes, as they are both proportional to $\sin \left(\frac{\alpha^{p \bar{p}}-\alpha^{p p}}{2} \tilde{\sigma}_{i n}\right)$. If this term is non-vanishing, but $\left(\alpha^{p \bar{p}}+\alpha^{p p}\right) \sigma_{i n}$ remains small, the above Odderon amplitude remains predominantly real, with a small, leading order linear in $\left(\alpha^{p \bar{p}}+\alpha^{p p}\right) \tilde{\sigma}_{i n}$ imaginary part. Given that $\alpha(s) \propto \rho_{0}(s)$ in the ReBB model, as detailed in Appendix B, and experimentally $\rho_{0}(s) \leq 0.15$ at LHC energies, the ReBB model Odderon amplitude is predominantly real at small values of $t$.

Equations (C.27, C.28) complete the proof, that the Odderon amplitude in the ReBB model vanishes if and only if the opacity parameters $\alpha(s)$ for elastic $p p$ and $p \bar{p}$ scattering are 

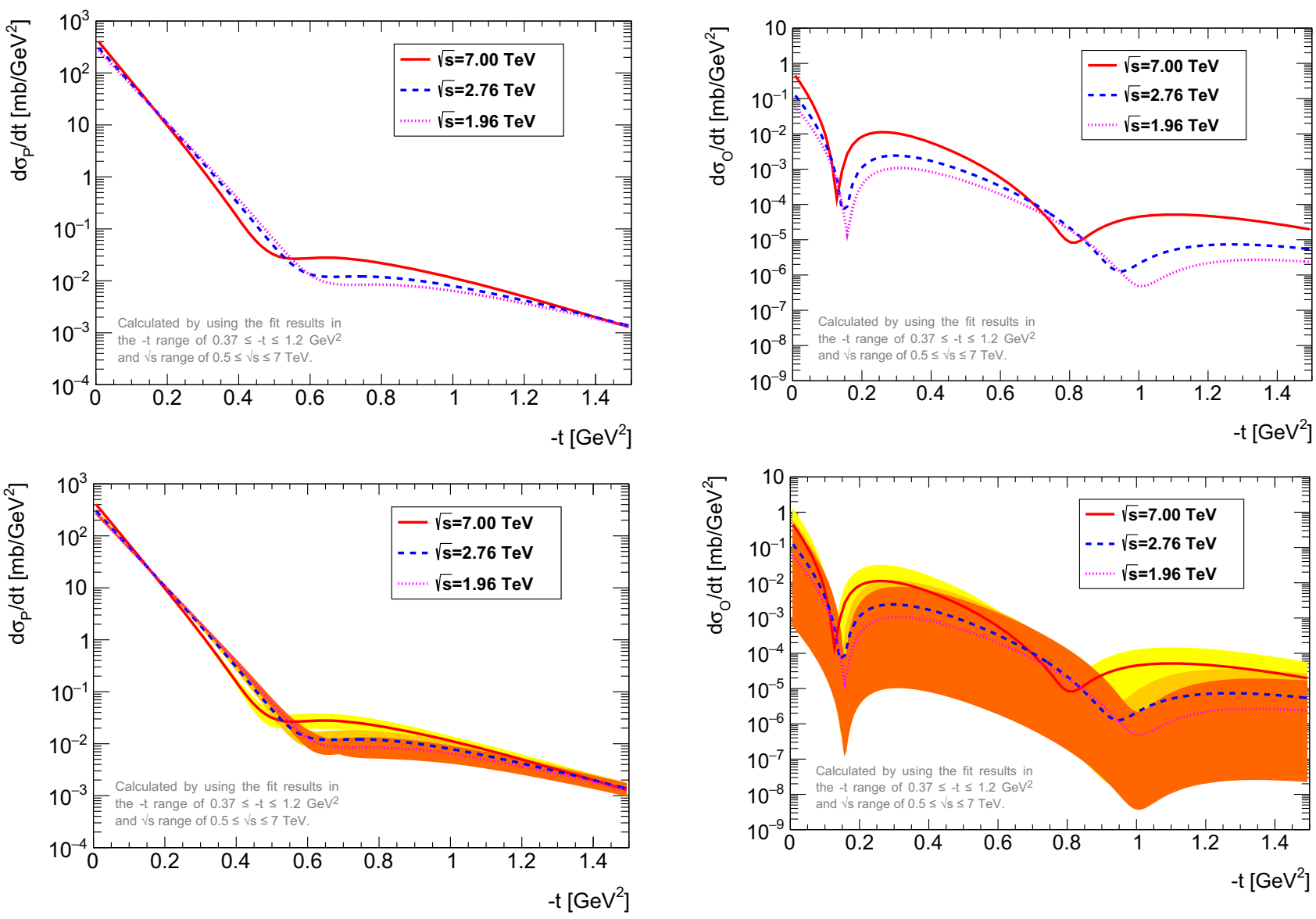

Fig. 21 The differential cross-section of Pomeron exchange, as calculated from ReBB model of Ref. [36], based on the log-linear excitation functions of $\alpha^{p p}(s), \alpha^{p \bar{p}}(s)$ and the scale parameters, $R_{q}(s), R_{d}(s)$, $R_{q d}(s)$, corresponding to Fig. 6 as summarized in Table 2 in the body of this manuscript. The top panel indicates the central values, while the lower panel includes our first estimates on the systematic errors of this reconstruction. The presented (over)estimates of the systematic error bands were obtained by neglecting the possible correlations between the parameters $p_{0}$ and $p_{1}$ for each of the excitation functions given in Table 2

equal, corresponding to $\alpha^{p \bar{p}}(s)=\alpha^{p p}(s)$. Note that these proofs are independent of the detailed calculations of the inelastic scattering probability $\tilde{\sigma}_{\text {in }}=\sigma_{\text {inel }}(s, b)$, hence they are valid both in the $p=(q, d)$ and in the $p=(q,(q, q))$ variant of the ReBB model. In fact they are valid for possible further generalized ReBB models as well, where for example the distribution of the scattering by quarks or diquarks is not assumed to be a Gaussian anymore, or if further parton contributions get resolved in a future paper.

In the following plots, we have evaluated the differential and total cross-sections of the Pomeron and the Odderon exchange, as well as the ratios of these differential crosssections, to determine the main properties of these processes with the help of the ReBB model of Ref. [36].

Fig. 22 Same as Fig. 21, but for the differential cross-section of the Odderon exchange

Figure 21 indicates the calculated differential crosssection for Pomeron exchange based on the fits presented in the body of this manuscript, utilizing the ReBB model of Ref. [36]. This result is based on Table 2, that summarizes the parameters of the excitation functions for the opacity parameters $\alpha^{p p}(s), \alpha^{p \bar{p}}(s)$ and the scale parameters, $R_{q}(s)$, $R_{d}(s), R_{q d}(s)$, corresponding to Fig. 6a-d in the body of this manuscript. The top panel indicates the central values for the differential cross-section of Pomeron exchange at various colliding energies $\sqrt{s}$, while the lower panel includes our first estimates on the systematic errors of this reconstruction. These first error band estimates were obtained by neglecting the possibly strong correlations between the parameters $p_{0}$ and $p_{1}$. These figures also indicate that Pomeron exchange does not lead to a pronounced diffractive minimum structure, in contrast to the experimental results for the diffractive minimum in elastic $p p$ collisions. This differential cross-section is more similar to the neck and shoulder type of structure, experimentally observed in elastic $p \bar{p}$ collisions, as discussed in the body of this manuscript.

Figure 22 is the same as Fig. 21, but for the C-odd Odderon exchange as evaluated from the ReBB model of Ref. [36]. 

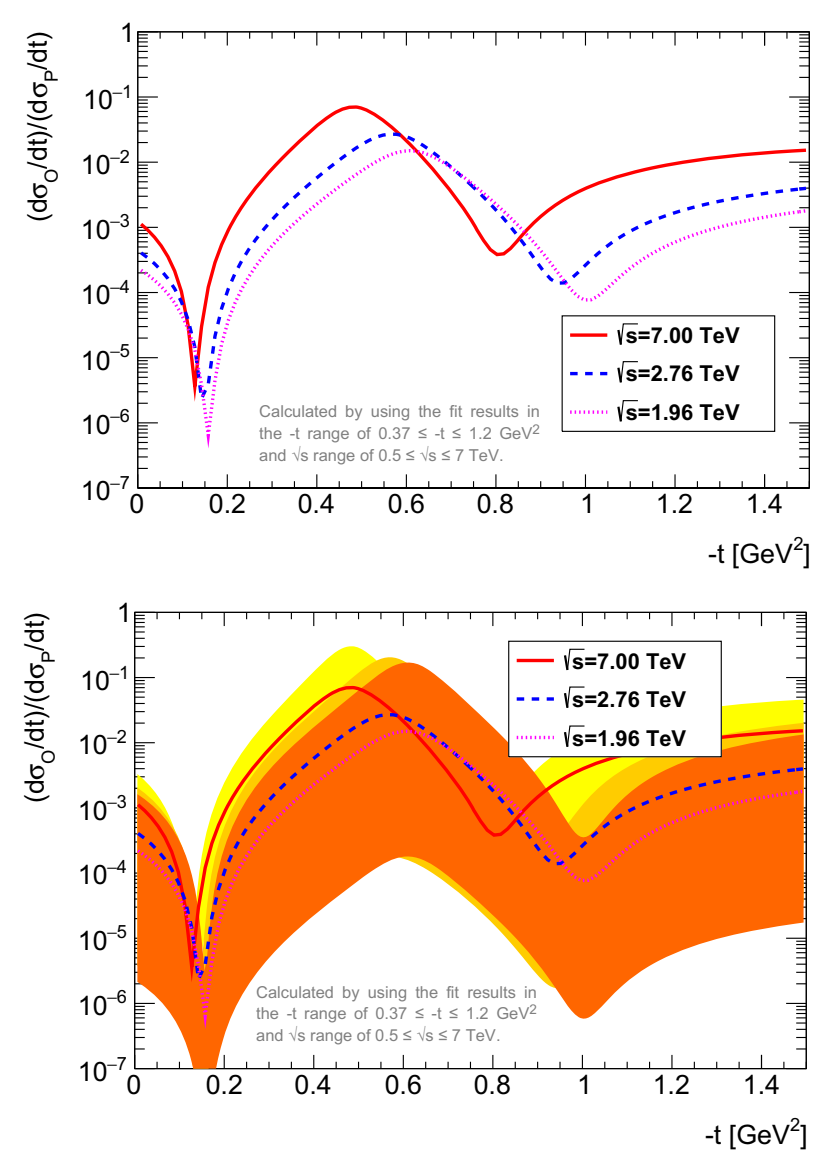

Fig. 23 Same as Fig. 21, but for the ratio of the differential crosssections of Odderon to Pomeron exchange

The top panel indicates the central values for the differential cross-section of Odderon exchange at various colliding energies $\sqrt{s}$ in the TeV domain, while the lower panel includes our first estimates on the systematic errors of this reconstruction, obtained by neglecting the possibly strong correlations between the parameters $p_{0}$ and $p_{1}$. These figures also indicate that Odderon exchange may lead even to two pronounced diffractive minima, in contrast to the experimental results for the diffractive minimum in elastic $p p$ collisions. However, the interference between the Pomeron and the Odderon exchange leads to a single well defined and experimentally resolvable diffractive minimum in elastic $p p$ collisions at the $\mathrm{TeV}$ scale.

Figure 23 indicates the ratio of the differential crosssections for Odderon to Pomeron exchange. This figure indicates that the Odderon contribution is important and relatively large in three kinematic regions: near to the $t=0$ optical point, near to the position of the diffractive minimum of elastic $p p$ collisions, $t_{d i p} \approx-0.5 \mathrm{GeV}^{2}$, and then at higher squared momentum transfer values, $-t \gtrsim 1 \mathrm{GeV}^{2}$. This figure also highlights with an explicit calculation, that the Odderon contribution to the dip region is correlated with
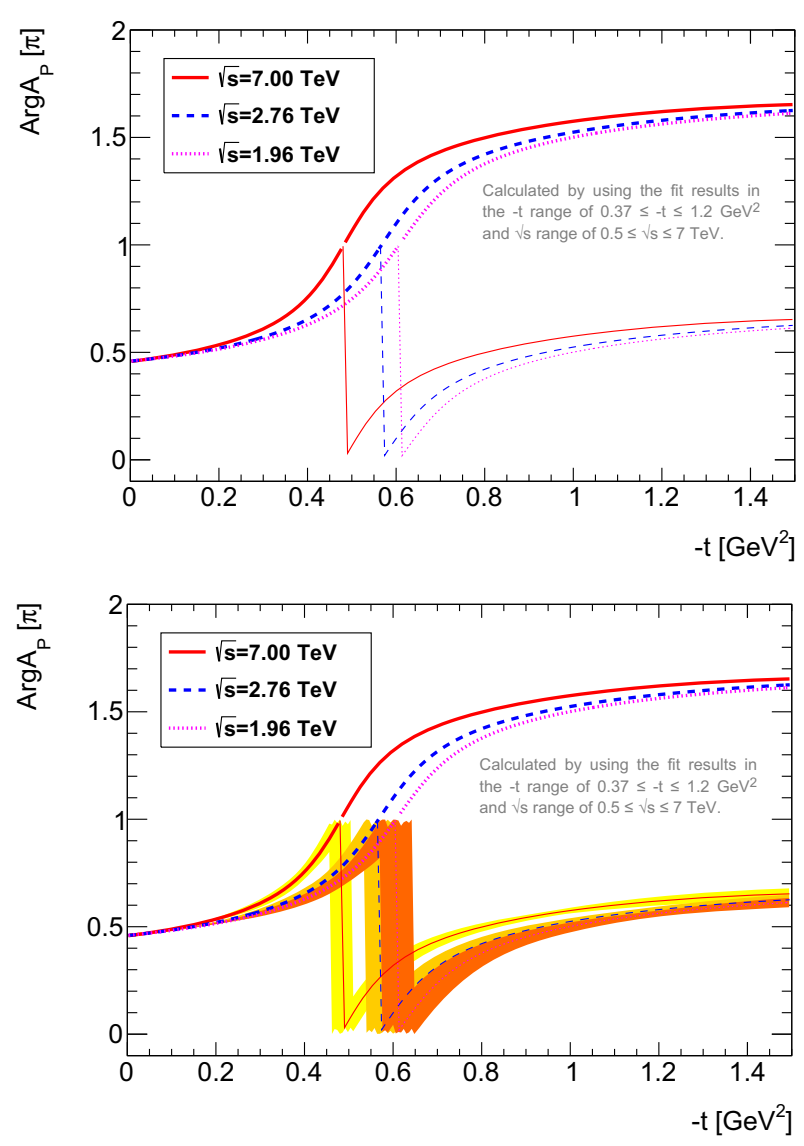

Fig. 24 Same as Fig. 21, but for the phase of the amplitude of Pomeron exchange

the Odderon contribution at $-t=0$, thus the Odderon signals at the dip region appear simultaneously with the Odderon signals at $-t=0$.

The last three figures characterize the modulus square of the amplitude for Pomeron and for Odderon exchanges in the ReBB model. A very important information, however, is included to the phase of these amplitudes, that are shown on the subsequent two figures.

The phase of Pomeron exchange is indicated on Fig. 24. This indicates that at low $-t$, the Pomeron contribution is predominantly imaginary, with a real component of the Pomeron exchange starting to be important near the diffractive minimum of elastic $p p$ collisions. On this plot, the principal value of the phase of the Pomeron (C-even) amplitude is indicated with a thin line, while the continuously varying phase evaluated from the multi-valued inverse tangent function is shown with the thick line.

The phase of Odderon exchange is indicated on Fig. 25. This indicates that at low $-t$, the Odderon contribution is predominantly real, with an imaginary component of the Odderon exchange starting to be important already at low $-t$ near to $0.1 \mathrm{GeV}^{2}$. This phase starts to change quickly and the Odd- 

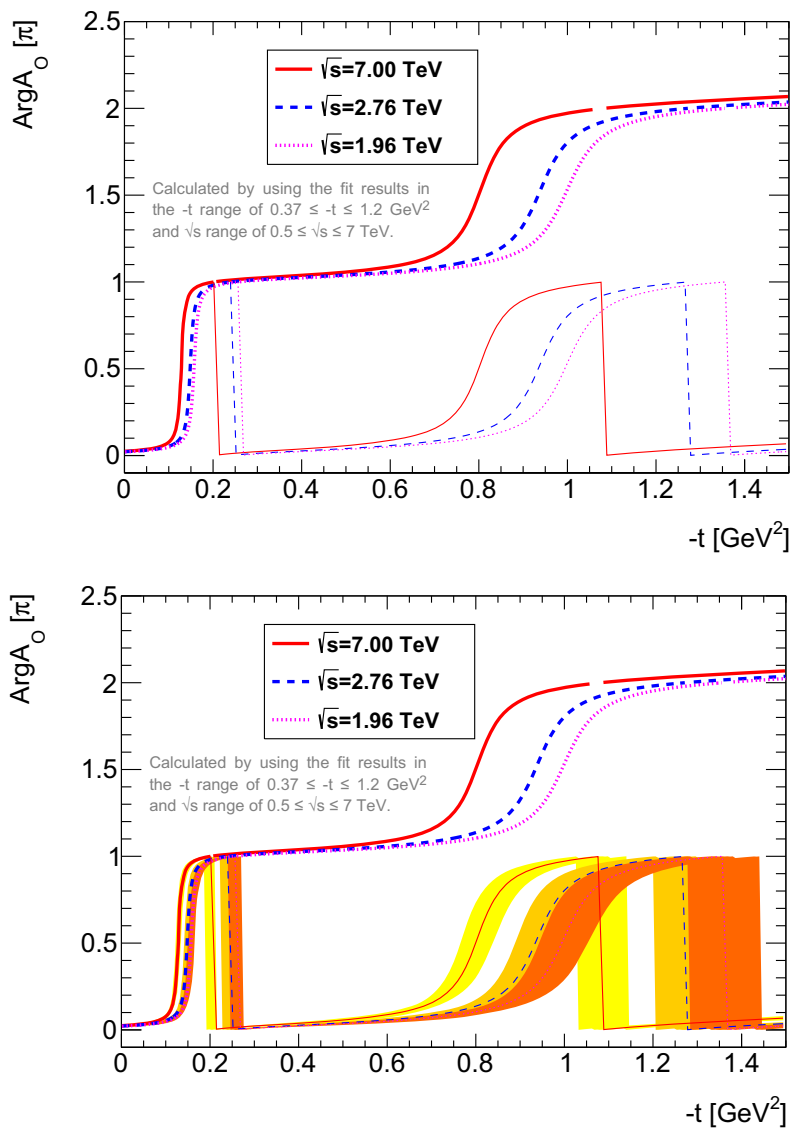

Fig. 25 Same as Fig. 21 but for the phase of the amplitude of Odderon exchange

eron becomes predominantly real again near the diffractive minimum of elastic $p p$ collisions. On this plot, the principal value of the phase of the Odderon (C-odd) amplitude is indicated with a thin line, while the continuously varying phase evaluated from the multi-valued inverse tangent function is shown with the thick line.

Figure 26 indicates the value of the real to imaginary ratio of the scattering amplitude $\rho_{0}(s)$ for elastic proton-proton, proton-antiproton scattering and for Pomeron exchange. Near to the optical point, all of these amplitudes are predominantly imaginary, with a small real part and with an even smaller C-odd contribution, that makes the $\rho_{0}(s)$ different for elastic $p p$ and $p \bar{p}$ collisions, due to the contribution of the C-odd Odderon exchange.

Figure 27 indicates the total cross-sections, as evaluated with the help of Eq. (2), for the elastic $p p$ and $p \bar{p}$ scattering as well as for the Pomeron exchange. The difference between the excitation functions for the total cross-sections of $p p$ and $p \bar{p}$ scattering seems to be less than the currently very small, of the order of $2 \%$ relative experimental error on the total cross-section measurements at LHC energies.

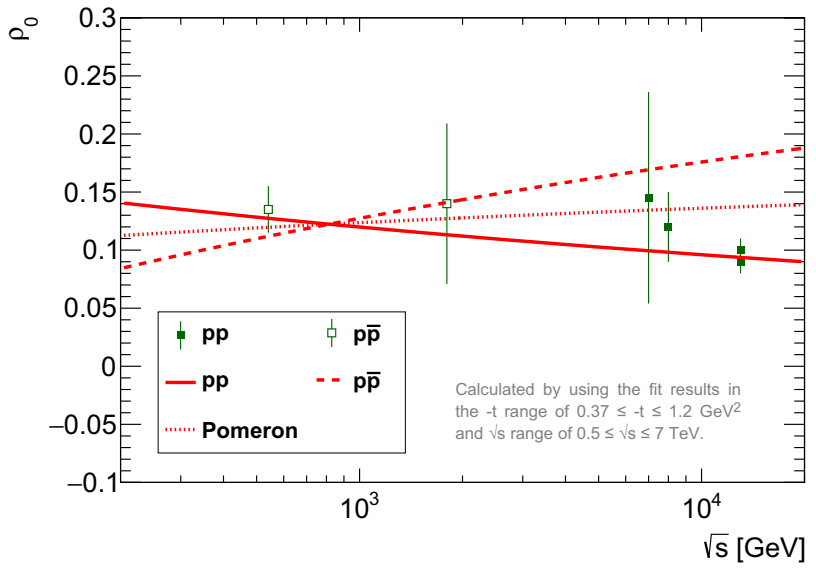

Fig. 26 The real to imaginary ratio $\rho_{0}(s)$ for elastic $p p$ and $p \bar{p}$ collisions and their Pomeron component evaluated from the log-linear excitation functions of the opacity parameters $\alpha^{p p}(s)$ and $\alpha^{p \bar{p}}(s)$ as well as that of the scale parameters, $R_{q}(s), R_{d}(s), R_{q d}(s)$, corresponding to Table 2

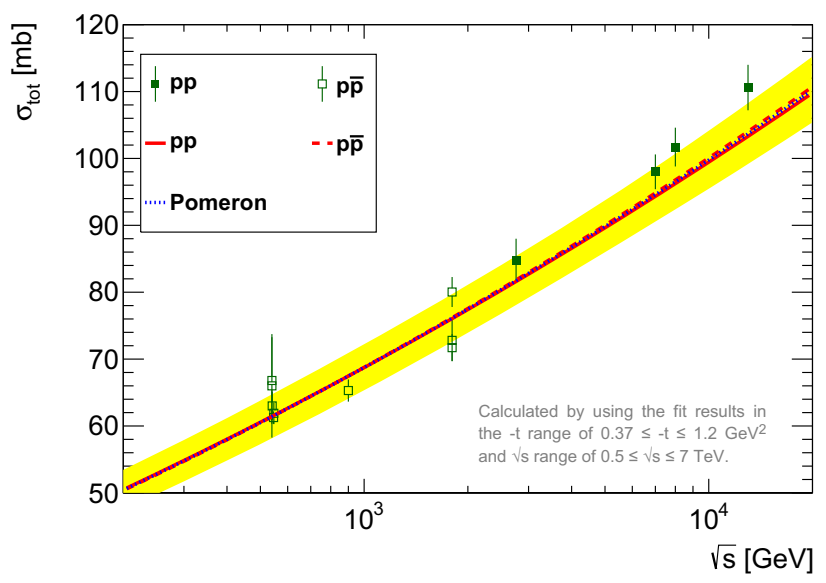

Fig. 27 Excitation function of the total cross-section for elastic $p p$, $p \bar{p}$ collisions and for the amplitude of Pomeron exchange, as evaluated from the log-linear excitation functions of the opacity parameters $\alpha^{p p}(s)$ and $\alpha^{p \bar{p}}(s)$ as well as that of the scale parameters, $R_{q}(s), R_{d}(s)$, $R_{q d}(s)$, corresponding to Table 2 . The yellow band indicates our conservative estimates on the systematic errors of the total cross-section of the Pomeron exchange

Finally, Fig. 28 indicates the total cross-section corresponding to the Odderon component of the scattering amplitude, as evaluated with the help of Eq. (2). This plot indicates that the Odderon cross-section starts to increase in the $\sqrt{s} \geq 1 \mathrm{TeV}$ energy domain, but the total cross-section of Odderon exchange is at least two orders of magnitude smaller than the total cross-section for elastic $p p$ scattering in the $\mathrm{TeV}$ energy scale. Actually we find $\sigma_{\text {tot }}^{\mathbb{Q}} \leq 0.7 \mathrm{mb}$ for $\sqrt{s} \leq 20$ $\mathrm{TeV}$.

Thus effectively, and within the framework of the ReBB model, we conclude that the Odderon occupies at least an 


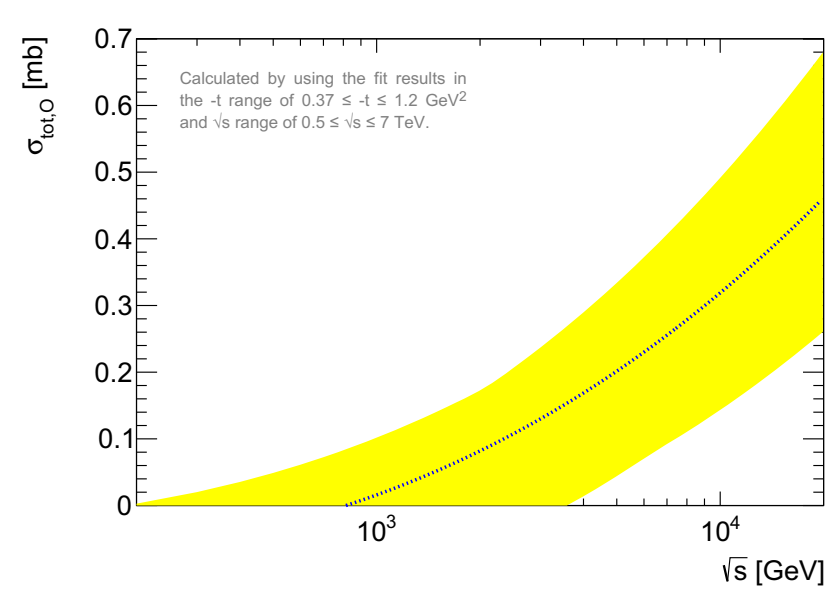

Fig. 28 Excitation function of the total cross-section obtained from the optical theorem using the ReBB model amplitude of Odderon exchange, as evaluated from the log-linear excitation functions of the opacity parameters $\alpha^{p p}(s)$ and $\alpha^{p \bar{p}}(s)$ as well as that of the scale parameters, $R_{q}(s), R_{d}(s), R_{q d}(s)$, corresponding to Table 2 . The yellow band indicates our conservative estimate on the systematic errors of the total cross-section of this Odderon exchange. The result indicates that total cross-section of the Odderon exchange is sharply increasing in the few $\mathrm{TeV}$ energy range, but it is two orders of magnitude smaller than the contribution of the Pomeron exchange that is dominant at the same energy scale

order of magnitude smaller radius, as compared to the effective size of the Pomeron exchange. Thus we support the observations of Refs. $[28,89,90]$, suggesting that the contribution of the Odderon exchange to the total $p p$ cross-section is rather small, of the order of $1 \mathrm{mb}$ or less, even at the currently available largest LHC energies. Nevertheless, we also find that this currently rather small effect is statistically significant, with a significance that is larger than the discovery threshold of $5 \sigma$, as detailed in the body of this manuscript.

\section{Appendix D: ISR energies and quadratic corrections to the excitation functions}

In this Appendix we investigate the stability of the obtained linear logarithmic energy dependencies of the ReBB model parameters, discussed in Sect. 5, for the case, when the energy range is extended towards lower values of $\sqrt{s}$. In order to do this, we refitted the ISR data [91] at all the five available collision energy $(\sqrt{s}=23.5,30.7,44.7,52.8$ and $62.5 \mathrm{GeV})$ in the squared momentum transfer range $0.8 \leq-t \leq 2.5$ $\mathrm{GeV}^{2}$ by using the $\chi^{2}$ definition determined by Eq. (6). The fits included the $t$-dependent (both vertical and horizontal) statistical (type A) and systematic (type B) errors, the normalization (type $\mathrm{C}$ ) error and the experimental values of the total cross section and the parameter $\rho_{0}$ with their total uncertainties [92]. We have also tested the stability of the fit results

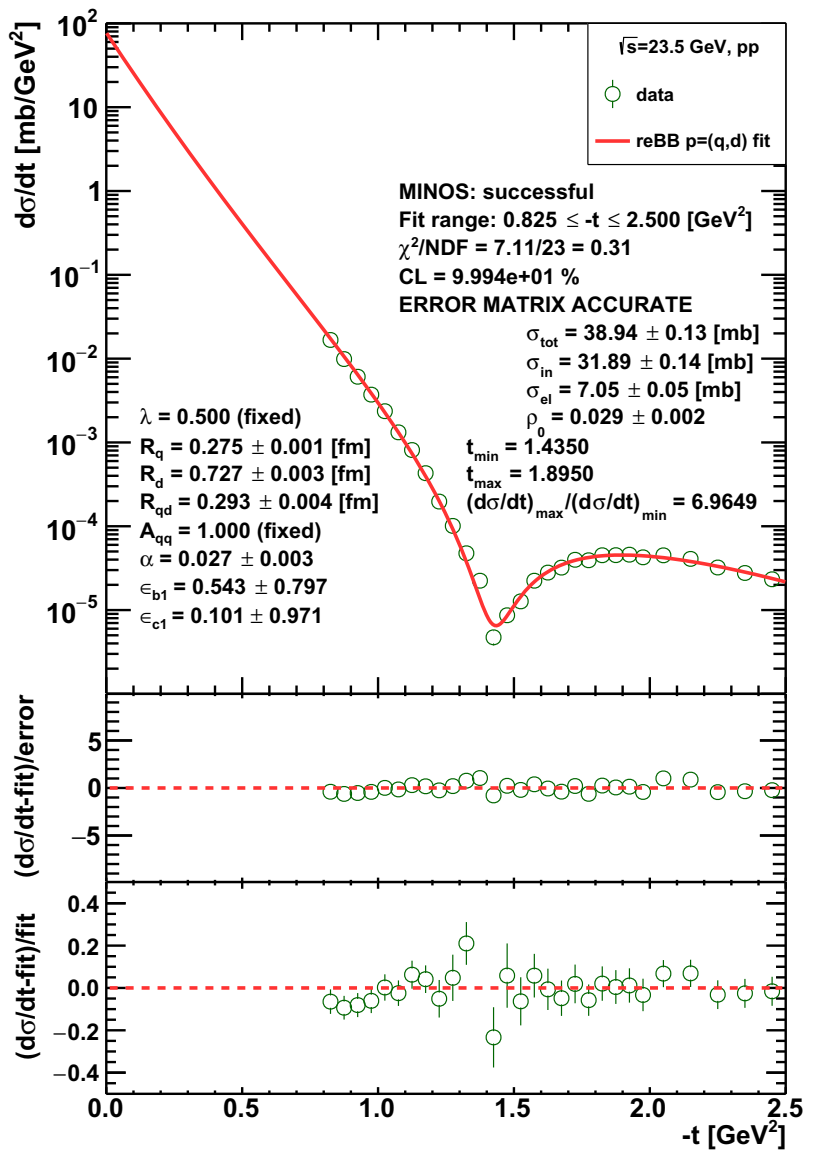

Fig. 29 The fit of the ReBB model to the $p p$ ISR $\sqrt{s}=23.5 \mathrm{GeV}$ data in the range of $0.8 \leq-t \leq 2.5 \mathrm{GeV}^{2}$ [92]. The fit includes the $t$-dependent statistical (type A) and systematic (type B) uncertainties, the normalization (type $C$ ) uncertainty and the experimental values of the total cross section and parameter $\rho_{0}$ with their full error according to Eq. (6). The fitted parameters are shown in the left bottom corner and their values are rounded up to three decimal digits

for small variations of the fit range or the fitting method. The only data set, where our results remained stable for the variation of the fit range around the selected range and for small variations of the fitting procedure, and where the obtained results were both statistically and physically acceptable fit results describing not only the differential cross-section but the measured value of the total cross-section $\sigma_{t o t}$ and the value of the real to imaginary ratio $\rho_{0}$ was the ISR dataset, measured at $\sqrt{s}=23.5 \mathrm{GeV}$. The result of this satisfactory fit is shown in Fig. 29. Our other results were similar to the results presented in Ref. [36] and particularly resulted in a rather fluctuating description of the excitation function of the $\alpha(s)$ at those ISR energies higher than $23.5 \mathrm{GeV}$. In the present study such fluctuating fits could not be used to establish the trends and the excitation functions.

Taking the restricted opportunities, we utilized the only reasonable ISR energy fit result, i.e., the result at $23.5 \mathrm{GeV}$ to cross-check the compatibility of the linear logarithmic trends 
obtained in Sect. 5 with the lower energy region. When the $\sqrt{s}=23.5 \mathrm{GeV}$ energy data are included to those summarized in Table 1, the energy dependence of the model parameters can be determined satisfactorily if model parameters are fitted one by one by applying a quadratic polynomial as a function of $\ln \left(s / s_{0}\right)$,

$$
\begin{aligned}
& P(s)=p_{0}+p_{1} \ln \left(s / s_{0}\right)+p_{2} \ln ^{2}\left(s / s_{0}\right), \\
& P \in\left\{R_{q}, R_{d}, R_{q d}, \alpha\right\},
\end{aligned}
$$

where $p_{0}, p_{1}, p_{2}$ are free parameters and $s_{0}$ is fixed at 1 $\mathrm{GeV}^{2}$. The obtained results are summarized in Fig. 30. The parameters of the excitation functions are indicated on the

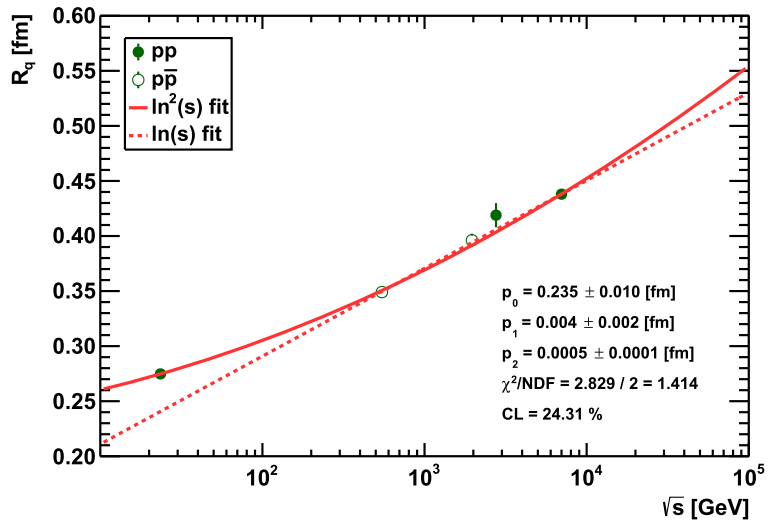

(a) Parameter $R_{q}$

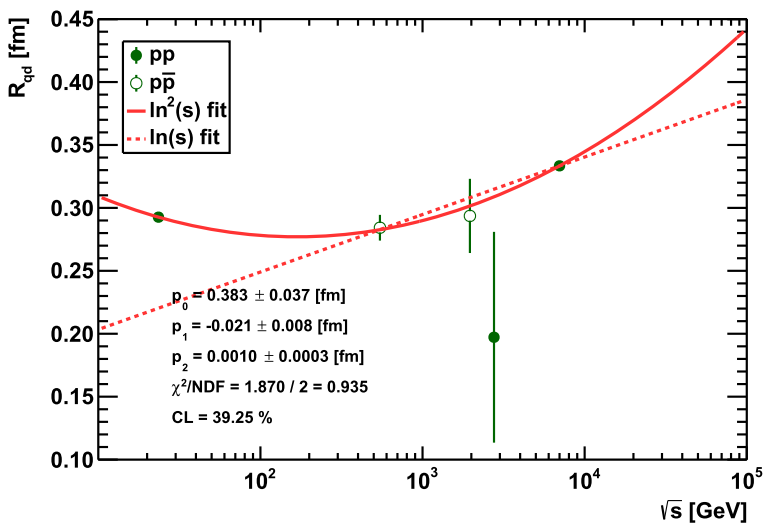

(c) Parameter $R_{q d}$

Fig. 30 The energy dependence of the parameters of the ReBB model, $R_{q}, R_{d}, R_{q d}$ and $\alpha$, taken from Fig. 29 and Table 1, determined by fitting a second order logarithmic polynomial, Eq. (D.1), to each of them one by one in the energy range of $23.5 \leq \sqrt{s} \leq 8000 \mathrm{GeV}$. As a comparison these figures also show the result of the fit in the energy range subplots of Fig. 30 and also summarized in Table 3. To fit the $\alpha$ parameter we used the same procedure described in Sect. 5 , i.e., utilizing also the measured and rescaled $\rho_{0}$ values. As seen in Figs. 31 and 32 the linear dependence of the ratio $\rho_{0}$ on the parameter $\alpha$ is satisfied at ISR energies as well.

In Fig. 30 the dotted curves show the result of the fits in the energy range of $546 \leq \sqrt{s} \leq 8000 \mathrm{GeV}$ with the linear logarithmic model determined by the parameters collected in Table 2 and discussed in Sect. 5. Investigating Fig. 30, one can conclude that although the energy dependence is not linear logarithmic if the data at the ISR energy region are

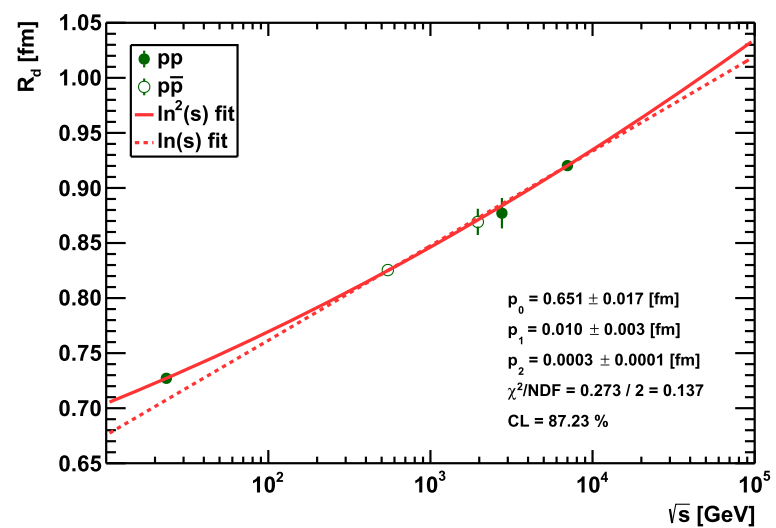

(b) Parameter $R_{d}$

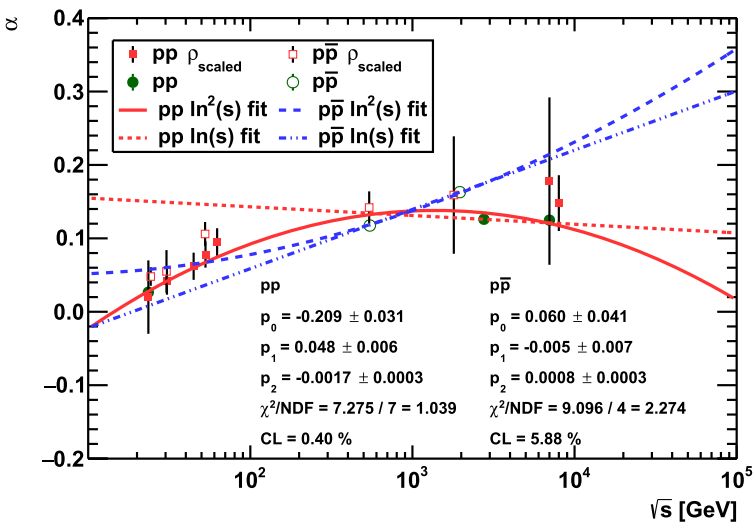

(d) Parameter $\alpha$

of $546 \leq \sqrt{s} \leq 8000 \mathrm{GeV}$ with the linear logarithmic model determined by the parameters collected in Table 2 . It is clear that allowing for quadratic corrections does not change significantly the linear trends in the kinematic range of $0.5 \leq \sqrt{s} \leq 8 \mathrm{TeV}$ 


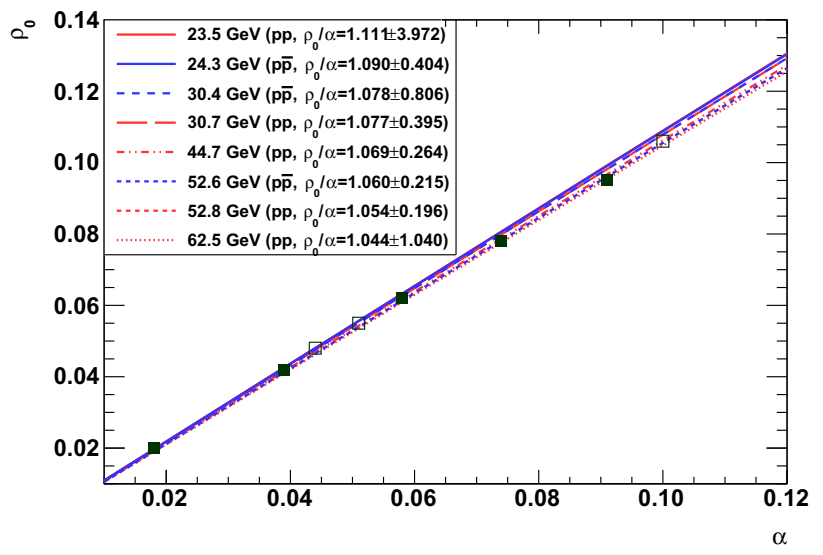

Fig. 31 Linearity between the ratio $\rho_{0}$ and the $\alpha$ parameter of the $\mathrm{ReBB}$ model in the few tens of $\mathrm{GeV}$ energy region calculated from the trends of the scale parameters, $R_{q}(s), R_{d}(s), R_{q d}(s)$, corresponding to Fig. 30a-c. The square shaped markers in the figure are positioned to the experimentally measured $\rho_{0}$ values. In the ISR energy range, the ratio $\rho_{0}(s) / \alpha(s)$ is in an excellent agreement with the analytic approximations given by Eq. (B.7) of Appendix B, as also illustrated on Fig. 32

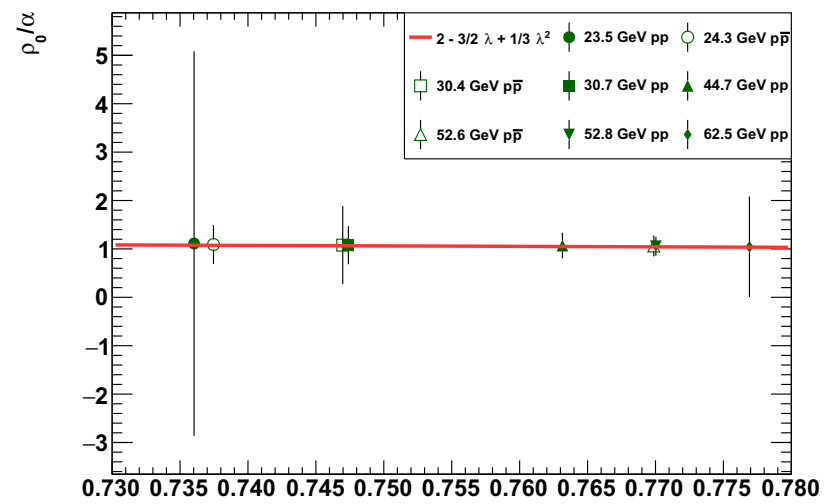

$\lambda$

Fig. 32 The dependence of $\rho_{0} / \alpha$ on $\lambda$ in the few tens of $\mathrm{GeV}$ energy range. Filled and empty symbols correspond to the $p p$ and $p \bar{p}$ cases, respectively. These values and the error-bars for $\rho / \alpha$ are obtained from the ReBB model fits by using the excitation functions of the scale parameters $R_{q}(s), R_{d}(s), R_{q d}(s)$, shown in Fig. 30a-c and summarized in Table 2 , as well as the experimentally measured ratio $\rho_{0}$ values. The red curve represents the analytic result, corresponding to Eq. (B.7) in Appendix B, showing a good agreement between the analytic considerations and the numerical results

included, the linear approximation in the energy region of $0.546 \leq \sqrt{s} \leq 8.0 \mathrm{TeV}$ is completely valid.

\section{References}

1. L. Lukaszuk, B. Nicolescu, A possible interpretation of pp rising total cross-sections. Lett. Nuovo Cim. 8, 405-413 (1973). https:// doi.org/10.1007/BF02824484

2. J. Bartels, L. Lipatov, G. Vacca, A New odderon solution in perturbative QCD. Phys. Lett. B 477, 178-186 (2000). https://doi.org/ 10.1016/S0370-2693(00)00221-5. arXiv:hep-ph/9912423
3. L.L. Jenkovszky, A.I. Lengyel, D.I. Lontkovskyi, The Pomeron and Odderon in elastic, inelastic and total cross sections at the LHC. Int. J. Mod. Phys. A 26, 4755-4771 (2011). https://doi.org/ 10.1142/S0217751X11054760. arXiv:1105.1202 [hep-ph]

4. A. Ster, L. Jenkovszky, T. Csörgó, Extracting the Odderon from $p p$ and $\bar{p} p$ scattering data. Phys. Rev. D 91(7), 074018 (2015). https://doi.org/10.1103/PhysRevD.91.074018. arXiv:1501.03860 [hep-ph]

5. A. Breakstone et al., A measurement of $\bar{p} p$ and $p p$ elastic scattering in the dip region at $\sqrt{s}=53-G e V$. Phys. Rev. Lett. 54, 2180 (1985). https://doi.org/10.1103/PhysRevLett.54.2180

6. TOTEM Collaboration, G. Antchev et al., First measurement of elastic, inelastic and total cross-section at $\sqrt{s}=13 \mathrm{TeV}$ by TOTEM and overview of cross-section data at LHC energies. Eur. Phys. J. C 79(2), 103 (2019). https://doi.org/10.1140/epjc/ s10052-019-6567-0. arXiv:1712.06153 [hep-ex]

7. TOTEM Collaboration, G. Antchev et al., First determination of the $\rho$ parameter at $\sqrt{s}=13 \mathrm{TeV}$ : probing the existence of a colourless three-gluon bound state. arXiv:1812.04732 [hep-ex]

8. TOTEM Collaboration, G. Antchev et al., Elastic differential cross-section measurement at $\sqrt{s}=13 \mathrm{TeV}$ by TOTEM. arXiv:1812.08283 [hep-ex]

9. TOTEM Collaboration, G. Antchev et al., Elastic differential crosssection $\mathrm{d} \sigma / \mathrm{d} t$ at $\sqrt{s}=2.76 \mathrm{TeV}$ and implications on the existence of a colourless 3-gluon bound state. arXiv:1812.08610 [hep-ex]

10. V.A. Khoze, A.D. Martin, M.G. Ryskin, Elastic proton-proton scattering at $13 \mathrm{TeV}$. Phys. Rev. D 97(3), 10.1103/PhysRevD.97.034019 (2018). https://doi.org/10.1103/PhysRevD.97. 034019. arXiv:1712.00325 [hep-ph]

11. A.P. Samokhin, V.A. Petrov, The stationary points and structure of high-energy scattering amplitude. Nucl. Phys. A 974, 45-55 (2018). https://doi.org/10.1016/j.nuclphysa.2018.03.009. arXiv: 1708.02879 [hep-ph]

12. T. Csörgő, R. Pasechnik, A. Ster, Odderon and proton substructure from a model-independent Lévy imaging of elastic $p p$ and $p \bar{p}$ collisions. Eur. Phys. J. C 79(1), 62 (2019). https://doi.org/10.1140/ epjc/s10052-019-6588-8. arXiv:1807.02897 [hep-ph]

13. M. Broilo, E.G.S. Luna, M.J. Menon, Forward elastic scattering and Pomeron models. Phys. Rev. D 98(7), 074006074006 (2018). https://doi.org/10.1103/PhysRevD.98.074006. arXiv:1807.10337 [hep-ph]

14. G. Pancheri, S. Pacetti, Y. Srivastava, Analysis and implications of precision near-forward TOTEM data. Phys. Rev. D 99(3), 034014 (2019). https://doi.org/10.1103/PhysRevD.99. 034014. arXiv:1811.00499 [hep-ph]

15. V.P. Gonçalves, P.V.R.G. Silva, The Phillips-Barger model for the elastic cross section and the Odderon. Eur. Phys. J. C 79(3), 237 (2019). https://doi.org/10.1140/epjc/s10052-019-6768-6. arXiv:1811.12250 [hep-ph]

16. O. Selyugin, J. Cudell, Odderon, HEGS model and LHC data. Acta Phys. Pol. Suppl. 12(4), 741 (2019). https://doi.org/10.5506/ APhysPolBSupp.12.741. arXiv:1810.11538 [hep-ph]

17. V.A. Khoze, A.D. Martin, M.G. Ryskin, Black disk, maximal Odderon and unitarity. Phys. Lett. B 780, 352-356 (2018). https://doi. org/10.1016/j.physletb.2018.03.025. arXiv:1801.07065 [hep-ph]

18. M. Broilo, E.G.S. Luna, M.J. Menon, Soft Pomerons and the forward LHC Data. Phys. Lett. B 781, 616-620 (2018). https://doi. org/10.1016/j.physletb.2018.04.045. arXiv:1803.07167 [hep-ph]

19. S.M. Troshin, N.E. Tyurin, Implications of the $\rho(s)$ measurements by TOTEM at the LHC. Mod. Phys. Lett. A 33(35), 1850206 (2018). https://doi.org/10.1142/S0217732318502061. arXiv:1805.05161 [hep-ph]

20. I.M. Dremin, Several effects unexplained by QCD. Universe 4(5), 65 (2018). https://doi.org/10.3390/universe4050065

21. E. Martynov, B. Nicolescu, Evidence for maximality of strong interactions from LHC forward data. Phys. Lett. B 786, 
207-211 (2018). https://doi.org/10.1016/j.physletb.2018.09.049. arXiv:1804.10139 [hep-ph]

22. E. Martynov, B. Nicolescu, Odderon effects in the differential cross-sections at Tevatron and LHC energies. Eur. Phys. J. C 79(6), 461 (2019). https://doi.org/10.1140/epjc/ s10052-019-6954-6. arXiv:1808.08580 [hep-ph]

23. Y.M. Shabelski, A.G. Shuvaev, Real part of $p p$ scattering amplitude in a additive quark model at LHC energies. Eur. Phys. J. C 78(6), 497 (2018). https://doi.org/10.1140/epjc/ s10052-018-5979-6. arXiv:1802.02812 [hep-ph]

24. V.A. Khoze, A.D. Martin, M.G. Ryskin, Elastic and diffractive scattering at the LHC. Phys. Lett. B 784, 192-198 (2018). https:// doi.org/10.1016/j.physletb.2018.07.054. arXiv:1806.05970 [hep$\mathrm{ph}]$

25. Y. Hagiwara, Y. Hatta, R. Pasechnik, J. Zhou, Spin-dependent Pomeron and Odderon in elastic proton-proton scattering. arXiv:2003.03680 [hep-ph]

26. C. Contreras, E. Levin, R. Meneses, M. Sanhueza, QCD Odderon: non linear evolution in the leading twist. arXiv:2004.04445 [hep$\mathrm{ph}]$

27. E. Gotsman, E. Levin, I. Potashnikova, A new parton model for the soft interactions at high energies: the Odderon. arXiv:2003.09155 [hep-ph]

28. E. Gotsman, E. Levin, I. Potashnikova, CGC/saturation approach: secondary Reggeons and $\rho=\operatorname{Re} / \mathrm{Im}$ dependence on energy. Phys. Lett. B 786, 472-476 (2018). https://doi.org/10.1016/j.physletb. 2018.10.017. arXiv:1807.06459 [hep-ph]

29. V.A. Petrov, A.P. Samokhin, Is there a hollow inside the proton? Int. J. Mod. Phys. Conf. Ser. 47, 1860097 (2018). https://doi.org/ 10.1142/S2010194518600972. arXiv:1801.03809 [hep-ph]

30. T. Csörgô, T. Novák, R. Pasechnik, A. Ster, I. Szanyi, Evidence of Odderon-exchange from scaling properties of elastic scattering at TeV energies. Eur. Phys. J . C 81(2), 180 (2021). https://doi.org/ 10.1140/epjc/s10052-021-08867-6. arXiv:1912.11968 [hep-ph]

31. T. Csörgô, T. Novák, R. Pasechnik, A. Ster, I. Szanyi, Proton holography-discovering Odderon from scaling properties of elastic scattering, in 49th International Symposium on Multiparticle Dynamics, vol. 4 (2020). arXiv:2004.07095 [hep-ph]

32. T. Csörgô, T. Novák, R. Pasechnik, A. Ster, I. Szanyi, Scaling of high-energy elastic scattering and the observation of Odderon (2020). https://doi.org/10.1142/9789811238406_0012. arXiv:2004.07318 [hep-ph]

33. F. Nemes, T. Csörgó, Detailed analysis of $p^{+} p$ elastic scattering data in the quark-diquark model of Bialas and Bzdak from $\sqrt{s}=$ 23.5 GeV to 7 TeV. Int. J. Mod. Phys. A 27, 1250175 (2012). https:// doi.org/10.1142/S0217751X12501758. arXiv:1204.5617 [hep-ph]

34. T. Csörgó, F. Nemes, Elastic scattering of protons from $\sqrt{s}=$ $23.5 \mathrm{GeV}$ to $7 \mathrm{TeV}$ from a generalized Bialas-Bzdak model. Int. J. Mod. Phys. A 29, 1450019 (2014). https://doi.org/10.1142/ S0217751X14500195. arXiv:1306.4217 [hep-ph]

35. T. Csörgó, R. Glauber, F. Nemes, Elastic proton-proton scattering from ISR to LHC energies, focusing on the dip region, in International Workshop on Low X Physics, vol. 11 (2013). arXiv:1311.2308 [hep-ph]

36. F. Nemes, T. Csörgő, M. Csanád, Excitation function of elastic pp scattering from a unitarily extended Bialas-Bzdak model. Int. J. Mod. Phys. A 30(14), 1550076 (2015). https://doi.org/10.1142/ S0217751X15500761. arXiv:1505.01415 [hep-ph]

37. A. Bialas, A. Bzdak, Wounded quarks and diquarks in heavy ion collisions. Phys. Lett. B 649, 263-268 (2007). https://doi.org/10. 1016/j.physletb.2007.04.014. arXiv:nucl-th/0611021 (Erratum: Phys. Lett. B 773, 681-681 (2017))

38. A. Bzdak, The diquark and elastic pion-proton scattering at high energies. Acta Phys. Pol. B 38, 2665-2672 (2007). arXiv:hep-ph/0701028
39. A. Bialas, A. Bzdak, Constituent quark and diquark properties from small angle proton-proton elastic scattering at high energies. Acta Phys. Pol. B 38, 159-168 (2007). arXiv:hep-ph/0612038

40. A. Bialas, A. Bzdak, Wounded quarks and diquarks in high energy collisions. Phys. Rev. C 77, 034908 (2008). https://doi.org/10. 1103/PhysRevC.77.034908. arXiv:0707.3720 [hep-ph]

41. R. Glauber, Cross-sections in deuterium at high-energies. Phys. Rev. 100, 242-248 (1955). https://doi.org/10.1103/PhysRev.100. 242

42. R. Glauber, G. Matthiae, High-energy scattering of protons by nuclei. Nucl. Phys. B 21, 135-157 (1970). https://doi.org/10.1016/ 0550-3213(70)90511-0

43. R.J. Glauber, Quantum optics and heavy ion physics. Nucl. Phys. A 774, 3-13 (2006). https://doi.org/10.1016/j.nuclphysa.2006.06. 009. arXiv:nucl-th/0604021

44. W. Czyz, L. Maximon, High-energy, small angle elastic scattering of strongly interacting composite particles. Ann. Phys. 52, 59-121 (1969). https://doi.org/10.1016/0003-4916(69)90321-2

45. D0 Collaboration, V.M. Abazov et al., Measurement of the differential cross section $d \sigma / d t$ in elastic $p \bar{p}$ scattering at $\sqrt{s}=1.96$ TeV. Phys. Rev. D 86, 012009 (2012). https://doi.org/10.1103/ PhysRevD.86.012009. arXiv:1206.0687 [hep-ex]

46. PHENIX Collaboration, A. Adare et al., Quantitative constraints on the opacity of hot partonic matter from semi-inclusive single high transverse momentum pion suppression in $\mathrm{Au}+\mathrm{Au}$ collisions at $\mathrm{s}(\mathrm{NN}) * *(1 / 2)=200-\mathrm{GeV}$. Phys. Rev. C 77, 064907 (2008). https:// doi.org/10.1103/PhysRevC.77.064907. arXiv:0801.1665 [nuclex]

47. TOTEM Collaboration, G. Antchev et al., Measurement of protonproton elastic scattering and total cross-section at $\sqrt{s}=7 \mathrm{TeV}$. EPL 101(2), 21002 (2013). https://doi.org/10.1209/0295-5075/ $101 / 21002$

48. UA4 Collaboration, R. Battiston et al., Proton-anti-proton elastic scattering at four momentum transfer up to $0.5-\mathrm{GeV}^{*} * 2$ at the CERN SPS Collider. Phys. Lett. B 127, 472 (1983). https://doi.org/ 10.1016/0370-2693(83)90296-4

49. UA4 Collaboration, M. Bozzo et al., Elastic scattering at the CERN SPS collider up to a four momentum transfer of 1.55GeV**2. Phys. Lett. B 155, 197-202 (1985). https://doi.org/10. 1016/0370-2693(85)90985-2

50. Particle Data Group Collaboration, M. Tanabashi et al., Rev. Part. Phys. Phys. Rev. D 98(3), 030001 (2018). https://doi.org/10.1103/ PhysRevD.98.030001

51. COMPETE Collaboration, J.R. Cudell, V.V. Ezhela, P. Gauron, K. Kang, Y.V. Kuyanov, S.B. Lugovsky, E. Martynov, B. Nicolescu, E.A. Razuvaev, N.P. Tkachenko, Benchmarks for the forward observables at RHIC, the Tevatron Run II and the LHC. Phys. Rev. Lett. 89, 201801 (2002). https://doi.org/10.1103/PhysRevLett.89. 201801. arXiv:hep-ph/0206172 [hep-ph]

52. F.J. Nemes, Elastic and total cross-section measurements by TOTEM: past and future. PoS DIS 2017, 059 (2018)

53. TOTEM Collaboration, G. Antchev et al., Measurement of protonproton inelastic scattering cross-section at $\sqrt{s}=7 \mathrm{TeV}$. EPL 101(2), 21003 (2013). https://doi.org/10.1209/0295-5075/101/21003

54. J. Kaspar, Soft diffraction at LHC. EPJ Web Conf. 172, 06005 (2018). https://doi.org/10.1051/epjconf/201817206005

55. TOTEM Collaboration, G. Antchev et al., Evidence for nonexponential elastic proton-proton differential cross-section at low $|t|$ and $\sqrt{s}=8 \mathrm{TeV}$ by TOTEM. Nucl. Phys. B 899, 527-546 (2015). https://doi.org/10.1016/j.nuclphysb.2015. 08.010. arXiv:1503.08111 [hep-ex]

56. TOTEM Collaboration, G. Antchev et al., Measurement of elastic pp scattering at $\sqrt{\mathrm{s}}=8 \mathrm{TeV}$ in the Coulomb-nuclear interference region: determination of the $\rho$-parameter and the total cross-section. Eur. Phys. J. C 76(12), 661 (2016). https://doi.org/ 10.1140/epjc/s10052-016-4399-8. arXiv:1610.00603 [nucl-ex] 
57. TOTEM Collaboration, T. Csörgó, Recent results from the CERN LHC experiment TOTEM: implications for Odderon exchange, in 48th International Symposium on Multiparticle Dynamics (ISMD 2018) Singapore, Singapore, September 3-7, 2018 (2019). arXiv:1903.06992 [hep-ex]

58. I. Dremin, Critical regime of proton elastic scattering at the LHC. JETP Lett. 99, 243-245 (2014). https://doi.org/10.1134/ S0021364014050075. arXiv:1401.3106 [hep-ph]

59. I.M. Dremin, Interaction region of high energy protons. Phys. Usp. 58(1), 61-70 (2015). https://doi.org/10.3367/UFNe.0185. 201501d.0065. arXiv:1406.2153 [hep-ph]

60. J.L. Albacete, A. Soto-Ontoso, Hot spots and the hollowness of proton-proton interactions at high energies. Phys. Lett. B 770, 149-153 (2017). https://doi.org/10.1016/j.physletb.2017.04.055. arXiv:1605.09176 [hep-ph]

61. S.M. Troshin, N.E. Tyurin, Experimental signatures of hadron asymptotics at the LHC. Int. J. Mod. Phys. A 32(17), 1750103 (2017). https://doi.org/10.1142/S0217751X17501032. arXiv:1704.00443 [hep-ph]

62. UA4/2 Collaboration, C. Augier et al., A Precise measurement of the real part of the elastic scattering amplitude at the S antip p S. Phys. Lett. B 316, 448-454 (1993). https://doi.org/10.1016/ 0370-2693(93)90350-Q

63. E710 Collaboration, N.A. Amos et al., Measurement of $\rho$, the ratio of the real to imaginary part of the $\bar{p} p$ forward elastic scattering amplitude, at $\sqrt{s}=1.8$-TeV. Phys. Rev. Lett. 68, 2433-2436 (1992). https://doi.org/10.1103/PhysRevLett.68.2433

64. E-811 Collaboration, C. Avila et al., The ratio, $\rho$, of the real to the imaginary part of the $\bar{p} p$ forward elastic scattering amplitude at $\sqrt{s}=1.8$-TeV. Phys. Lett. B 537, 41-44 (2002). https://doi.org/10. 1016/S0370-2693(02)01908-1

65. TOTEM Collaboration, G. Antchev et al., Luminosity-independent measurements of total, elastic and inelastic cross-sections at $\sqrt{s}=7$ TeV. EPL 101(2), 21004 (2013). https://doi.org/10.1209/ 0295-5075/101/21004

66. UA4 Collaboration, D. Bernard et al., Large T Elastic Scattering at the CERN SPS Collider at $\sqrt{s}=630-\mathrm{GeV}$. Phys. Lett. B 171, 142-144 (1986). https://doi.org/10.1016/0370-2693(86)91014-2

67. E-710 Collaboration, N.A. Amos et al., $\bar{p} p$ Elastic Scattering at $\sqrt{s}=1.8-\mathrm{TeV}$ from $|\mathrm{t}|=0.034-\mathrm{GeV} / \mathrm{c}^{2}$ to $0.65-$ $\mathrm{GeV} / \mathrm{c}^{2}$. Phys. Lett. B 247, 127-130 (1990). https://doi.org/10. 1016/0370-2693(90)91060-O

68. TOTEM Collaboration, G. Antchev et al., Luminosity-independent measurement of the proton-proton total cross section at $\sqrt{s}=8$ TeV. Phys. Rev. Lett. 111(1), 012001 (2013). https://doi.org/10. 1103/PhysRevLett.111.012001

69. A. Donnachie, P. Landshoff, $p$ p and anti-p p elastic scattering. Nucl. Phys. B 231, 189-204 (1984). https://doi.org/10.1016/ 0550-3213(84)90283-9

70. I. Szanyi, N. Bence, L. Jenkovszky, New physics from TOTEM's recent measurements of elastic and total cross sections. J. Phys. G 46(5), 055002 (2019). https://doi.org/10.1088/1361-6471/ab1205. arXiv:1808.03588 [hep-ph]

71. A. Donnachie, P. Landshoff, The coupling of the Odderon. Nucl. Phys. B 348, 297-309 (1991). https://doi.org/10.1016/ 0550-3213(91)90520-8

72. L. Van Hove, A phenomenological discussion of inelastic collisions at high energies. Il Nuovo Cimento (1955-1965) 28(4), 798-817 (1963)

73. L. Van Hove, High-energy collisions of strongly interacting particles. Rev. Mod. Phys. 36, 655-665 (1964). https://doi.org/10.1103/ RevModPhys.36.655. (216 (1964))

74. S. Troshin, N. Tyurin, Beyond the black disk limit. Phys. Lett. B 316(1), 175-177 (1993). https://doi.org/10.1016/ 0370-2693(93)90675-8
75. E.R. Arriola, W. Broniowski, Proton-proton on shell optical potential at high energies and the hollowness effect. Few Body Syst. 57(7), 485-490 (2016). https://doi.org/10.1007/ s00601-016-1095-z. arXiv:1602.00288 [hep-ph]

76. E.R. Arriola, W. Broniowski, Proton-proton hollowness at the LHC from inverse scattering. Phys. Rev. D 95(7), 074030 (2017). https:// doi.org/10.1103/PhysRevD.95.074030. arXiv:1609.05597 [nuclth]

77. W. Broniowski, E.R. Arriola, Hollowness in pp scattering. Acta Phys. Pol. B 48, 927 (2017). https://doi.org/10.5506/APhysPolB. 48.927. arXiv:1704.03271 [hep-ph]

78. W. Broniowski, E.R. Arriola, Hollowness in pp scattering at the LHC. Acta Phys. Pol. Suppl. 10, 1203 (2017). https://doi.org/10. 5506/APhysPolBSupp.10.1203. arXiv:1708.00402 [nucl-th]

79. W. Broniowski, E.R. Arriola, Hollowness in pp scattering at the LHC. Acta Phys. Pol. Suppl. 10, 1203 (2017). https://doi.org/10. 1103/PhysRevD.98.074012. arXiv:1806.04756 [hep-ph]

80. S.M. Troshin, N.E. Tyurin, Reflective scattering from unitarity saturation. Int. J. Mod. Phys. A 22, 4437-4449 (2007). https://doi. org/10.1142/S0217751X0703697X. arXiv:hep-ph/0701241

81. S.M. Troshin, N.E. Tyurin, Reflective scattering at the LHC and two-scale structure of a proton. EPL 129(3), 31002 (2020). https:// doi.org/10.1209/0295-5075/129/31002. arXiv:2001.06260 [hep$\mathrm{ph}]$

82. S.M. Troshin, N.E. Tyurin, Increasing effective intensity of soft strong interactions. Int. J. Mod. Phys. A 35(27), 2050171 (2020). https://doi.org/10.1142/S0217751X20501717. arXiv:2008.06355 [hep-ph]

83. S.D. Campos, V.A. Okorokov, Hollowness effect and entropy in high energy elastic scattering. Phys. Scr. 95(9), 095305 (2020). https://doi.org/10.1088/1402-4896/abaf8d. arXiv:1807.02061 [hep-ph]

84. S.D. Campos, An approach to the leading Regge pole. Phys. Scr. 95(6), 065302 (2020). https://doi.org/10.1088/1402-4896/ab7aab. arXiv:2002.12091 [hep-ph]

85. T. Csörgő, R. Pasechnik, A. Ster, Proton structure and hollowness from Lévy imaging of $p p$ elastic scattering. Eur. Phys. J. C 80(2), 126 (2020). https://doi.org/10.1140/epjc/ s10052-020-7681-8. arXiv:1910.08817 [hep-ph]

86. R. Glauber, J. Velasco, Multiple diffraction theory of $p \bar{p}$ scattering at 546-GeV. Phys. Lett. B 147, 380-384 (1984). https://doi.org/10. 1016/0370-2693(84)90137-0

87. I. Andreev, I. Dremin, I. Gramenitsky, High-energy p-p large angle scattering. Nucl. Phys. B 10, 137-144 (1969). https://doi.org/10. 1016/0550-3213(69)90291-0

88. I. Dremin, Some new discoveries at colliders. Usp. Fiz. Nauk 188(4), 437-445 (2018). https://doi.org/10.3367/UFNe.2018.01. 038284

89. J. Bartels, C. Contreras, G.P. Vacca, The Odderon in QCD with running coupling. JHEP 04, 183 (2020). https://doi.org/10.1007/ JHEP04(2020)183. arXiv:1910.04588 [hep-th]

90. E. Gotsman, E. Levin, I. Potashnikova, A new parton model for the soft interactions at high energies: two channel approximation. arXiv:1908.05673 [hep-ph]

91. E. Nagy et al., Measurements of elastic proton proton scattering at large momentum transfer at the CERN intersecting storage rings. Nucl. Phys. B 150, 221-267 (1979). https://doi.org/10.1016/ 0550-3213(79)90301-8

92. U. Amaldi, K.R. Schubert, Impact parameter interpretation of proton proton scattering from a critical review of all ISR data. Nucl. Phys. B 166, 301-320 (1980). https://doi.org/10.1016/ 0550-3213(80)90229-1 